TRANSACTIONS OF THE

AMERICAN MATHEMATICAL SOCIETY

Volume 361, Number 9, September 2009, Pages 4683-4720

S 0002-9947(09)04771-0

Article electronically published on April 10, 2009

\title{
MULTIDIMENSIONAL OPERATOR MULTIPLIERS
}

\author{
K. JUSCHENKO, I. G. TODOROV, AND L. TUROWSKA
}

\begin{abstract}
We introduce multidimensional Schur multipliers and characterise them, generalising well-known results by Grothendieck and Peller. We define a multidimensional version of the two-dimensional operator multipliers studied recently by Kissin and Shulman. The multidimensional operator multipliers are defined as elements of the minimal tensor product of several $C^{*}$-algebras satisfying certain boundedness conditions. In the case of commutative $C^{*}$ algebras, the multidimensional operator multipliers reduce to continuous multidimensional Schur multipliers. We show that the multipliers with respect to some given representations of the corresponding $C^{*}$-algebras do not change if the representations are replaced by approximately equivalent ones. We establish a non-commutative and multidimensional version of the characterisations by Grothendieck and Peller which shows that universal operator multipliers can be obtained as certain weak limits of elements of the algebraic tensor product of the corresponding $C^{*}$-algebras.
\end{abstract}

\section{INTRODUCTION}

A bounded function $\varphi: \mathbb{N} \times \mathbb{N} \rightarrow \mathbb{C}$ is called a Schur multiplier if $\left(\varphi(i, j) a_{i j}\right)$ is the matrix of a bounded linear operator on $\ell^{2}$ whenever $\left(a_{i j}\right)$ is such. The study of Schur multipliers was initiated by Schur in the early 20th century. A characterisation of these objects was given by A. Grothendieck in his Résumé [16, where he showed that Schur multipliers are precisely the functions $\varphi$ of the form $\varphi(i, j)=\sum_{k=1}^{\infty} a_{k}(i) b_{k}(j)$, where $a_{k}, b_{k}: \mathbb{N} \rightarrow \mathbb{C}$ are such that $\sup _{i} \sum_{k=1}^{\infty}\left|a_{k}(i)\right|^{2}<$ $\infty$ and $\sup _{j} \sum_{k=1}^{\infty}\left|b_{k}(j)\right|^{2}<\infty$. Schur multipliers have had many important applications in analysis; see e.g. 2], 10] and 26]. One of the forms of the celebrated Grothendieck inequality can be given in terms of these objects [26].

One of the most important developments in analysis in recent years has been "quantisation" 12, starting with the advent of the theory of operator spaces in the 1980s in the work of Blecher, Effros, Haagerup, Paulsen, Pisier, Ruan, Sinclair and many others, and based on Arveson's pioneering work in the 1970s. Operator space (or non-commutative) versions are presently being found for many results in classical Banach space theory [7, 22, 27. A construction underlying many of the developments in Operator Space Theory is the Haagerup tensor product, as well as its weak counterpart, the weak* Haagerup tensor product [8] and its generalisation, the extended Haagerup tensor product [15]. Grothendieck's characterisation can be formulated by saying that the set of Schur multipliers coincides with the extended

Received by the editors July 5, 2007.

2000 Mathematics Subject Classification. Primary 46L07; Secondary 47L25.

Key words and phrases. Multiplier, $C^{*}$-algebra, multidimensional. 
(or the weak*) Haagerup tensor product $\ell^{\infty} \otimes_{e h} \ell^{\infty}$ of the space $\ell^{\infty}$ of all bounded complex sequences with itself.

Schur multipliers are elements of the commutative von Neumann algebra $\ell^{\infty}(\mathbb{N} \times \mathbb{N})$, or equivalently of the (von Neumann) tensor product of (the commutative von Neumann algebra) $\ell^{\infty}$ with itself. Subsequently, they form a commutative algebra themselves. Their quantisation was initiated by Kissin and Shulman in [21. Suppose that $\mathcal{A}$ and $\mathcal{B}$ are $C^{*}$-algebras and $\pi$ and $\rho$ their representations on $H$ and $K$, respectively. The Hilbert space tensor product $H \otimes K$ can be naturally identified with the Hilbert space $\mathcal{C}_{2}\left(H^{\mathrm{d}}, K\right)$ of Hilbert-Schmidt operators from the dual $H^{\mathrm{d}}$ of $H$ into $K$. It follows that $\pi$ and $\rho$ give rise to a representation $\sigma_{\pi, \rho}$ of the minimal tensor product $\mathcal{A} \otimes \mathcal{B}$ of $\mathcal{A}$ and $\mathcal{B}$ on $\mathcal{C}_{2}\left(H^{\mathrm{d}}, K\right)$. Kissin and Shulman call an element $\varphi \in \mathcal{A} \otimes \mathcal{B}$ a $(\pi, \rho)$-multiplier if $\sigma_{\pi, \rho}(\varphi)$ is bounded in the norm of $\mathcal{C}_{2}\left(H^{\mathrm{d}}, K\right)$ induced by its inclusion into the algebra $\mathcal{B}\left(H^{\mathrm{d}}, K\right)$ of all bounded operators from $H^{\text {d }}$ into $K$. In [21], they study two sets of problems: the dependence of $(\pi, \rho)$ multipliers on $\pi$ and $\rho$ and the description of the norm of an operator multiplier. Most of their results are established in the more general setting of symmetrically normed ideals.

Assume that $\mathcal{A}$ and $\mathcal{B}$ are commutative, say $\mathcal{A}=C_{0}(X)$ and $\mathcal{B}=C_{0}(Y)$, for some locally compact Hausdorff spaces $X$ and $Y$, and that the representations $\pi$ and $\rho$ arise from some spectral measures on $X$ and $Y$. The notion of a $(\pi, \rho)$-multiplier is in this case closely related to that of double operator integrals introduced and developed by Birman and Solomyak 3, 4, 5, 6 in connection with various problems of Mathematical Physics and in particular of Perturbation Theory. If $(X, \mathcal{E})$ and $(Y, \mathcal{F})$ are spectral measures on the Hilbert spaces $H$ and $K$, they defined the double operator integral

$$
I_{\psi}(T)=\int_{X \times Y} \psi(x, y) d \mathcal{E}(x) T d \mathcal{F}(y),
$$

for every bounded measurable function $\psi$ and every operator $T$ from the HilbertSchmidt class $\mathcal{C}_{2}(H, K)$. A function $\psi$ is called a Schur multiplier with respect to $\mathcal{E}$ and $\mathcal{F}$ if $I_{\psi}$ can be extended to a bounded linear operator on the space $\left(\mathcal{B}(H, K),\|\cdot\|_{\text {op }}\right)$ of bounded operators from $H$ to $K$, that is, if there exists $C>0$ such that $\left\|I_{\psi}(T)\right\|_{\text {op }} \leq C\|T\|_{\text {op }}$ for all $T \in \mathcal{C}_{2}(H, K)$. Peller [24] (see also [19]) characterised Schur multipliers with respect to $\mathcal{E}$ and $\mathcal{F}$ in several ways. In particular, he showed that the space of Schur multipliers with respect to $\mathcal{E}$ and $\mathcal{F}$ coincides with the extended Haagerup tensor product $L^{\infty}(X) \otimes_{e h} L^{\infty}(Y)$ and the integral projective tensor product $L^{\infty}(X) \hat{\otimes}_{i} L^{\infty}(Y)$.

Several attempts were made to generalise the Birman-Solomyak theory to the case of multiple operator integrals [23, 31, 30. Such integrals appear, for instance, in the study of differentiability of functions of operators depending on a parameter. A recent definition of multiple operator integrals by Peller in [25] is based on the integral projective tensor product. For some fixed spectral measures $\left(X_{1}, \mathcal{E}_{1}\right), \ldots,\left(X_{n}, \mathcal{E}_{n}\right)$ on Hilbert spaces $H_{1}, \ldots, H_{n}$, he defines $I_{\psi}\left(T_{1}, \ldots, T_{n-1}\right)$ to be equal to

$$
\int_{X_{1} \times \ldots \times X_{n}} \psi\left(x_{1}, \ldots, x_{n}\right) d \mathcal{E}_{1}\left(x_{1}\right) T_{1} d \mathcal{E}_{2}\left(x_{2}\right) \ldots T_{n-1} d \mathcal{E}_{n}\left(x_{n}\right),
$$


where $\psi \in L^{\infty}\left(X_{1}\right) \hat{\otimes}_{i} \ldots \hat{\otimes}_{i} L^{\infty}\left(X_{n}\right)$ and $T_{1}, \ldots, T_{n-1}$ are bounded linear operators, and shows that

$$
\left\|I_{\psi}\left(T_{1}, \ldots, T_{n-1}\right)\right\|_{\mathrm{op}} \leq\|\psi\|_{i}\left\|T_{1}\right\|_{\mathrm{op}} \ldots\left\|T_{n-1}\right\|_{\mathrm{op}},
$$

where $\|\psi\|_{i}$ denotes the integral projective tensor norm of $\psi$. If the spectral measures are multiplicity free and $T_{1}, \ldots, T_{n-1}$ are Hilbert-Schmidt operators with kernels $f_{1}, \ldots, f_{n-1}$, respectively, then $I_{\psi}\left(T_{1}, \ldots, T_{n-1}\right)$ is a Hilbert-Schmidt operator with kernel $S_{\psi}\left(f_{1}, \ldots, f_{n-1}\right) \in L^{2}\left(X_{1} \times X_{n}\right)$ equal to

$$
\int \psi\left(x_{1}, \ldots, x_{n}\right) f_{1}\left(x_{1}, x_{2}\right) \ldots f_{n-1}\left(x_{n-1}, x_{n}\right) d \mathcal{E}_{2}\left(x_{2}\right) \ldots d \mathcal{E}_{n-1}\left(x_{n-1}\right) .
$$

This was the starting point for our definition of multidimensional Schur multipliers in Section 3, Let $\left(X_{i}, \mu_{i}\right), i=1, \ldots, n$, be standard $\sigma$-finite measure spaces and $\Gamma\left(X_{1}, \ldots, X_{n}\right)=L^{2}\left(X_{1} \times X_{2}\right) \odot L^{2}\left(X_{2} \times X_{3}\right) \odot \ldots \odot L^{2}\left(X_{n-1} \times X_{n}\right)$ be the algebraic tensor product of the corresponding $L^{2}$-spaces equipped with the projective tensor norm, where each of the $L^{2}$-spaces is equipped with its $L^{2}$-norm. An element $\psi \in L^{\infty}\left(X_{1} \times \cdots \times X_{n}\right)$ determines a bounded linear map $S_{\psi}$ from $\Gamma\left(X_{1}, \ldots, X_{n}\right)$ to $L^{2}\left(X_{1}, X_{n}\right)$ given on elementary tensors $f_{1} \otimes \ldots \otimes f_{n} \in \Gamma\left(X_{1}, \ldots, X_{n}\right)$ by (11) (where the integration is now with respect to $\mu_{i}$ instead of $\mathcal{E}_{i}$ ). This definition extends the multivariable Schur product defined by Effros and Ruan in their study of completely bounded multipliers of multivariable Fourier algebras of discrete groups 13. On the other hand, for any measure spaces $(X, \mu)$ and $(Y, \nu)$, the space $L^{2}(X \times Y)$ can be identified with the class of all Hilbert-Schmidt operators from $L^{2}(X)$ to $L^{2}(Y)$; to each $f \in L^{2}(X \times Y)$ there corresponds the operator $T_{f}$ given by $T_{f} \xi(y)=\int_{X} f(x, y) \xi(x) d \mu(x), \xi \in L^{2}(X)$. Using this identification, one can equip the space $L^{2}(X \times Y)$ with the opposite operator space structure arising from the inclusion of $L^{2}(X \times Y)$ into $\mathcal{B}\left(L^{2}(X), L^{2}(Y)\right)$. We further equip $\Gamma\left(X_{1}, \ldots, X_{n}\right)$ with the Haagerup tensor norm $\|\cdot\|_{\mathrm{h}}$, where the $L^{2}$-spaces are given their opposite operator space structure described above, and say that an element $\psi \in L^{\infty}\left(X_{1} \times \ldots \times X_{n}\right)$ is a Schur multiplier (with respect to $\mu_{1}, \ldots, \mu_{n}$ ) if there exists $C>0$ such that

$$
\left\|S_{\psi}(\Phi)\right\|_{\mathrm{op}} \leq C\|\Phi\|_{\mathrm{h}}, \text { for all } \Phi \in \Gamma\left(X_{1}, \ldots, X_{n}\right) .
$$

Using a generalisation of a result of Smith [28] on the complete boundedness of certain bounded bimodule maps to the case of multilinear modular maps, we obtain a characterisation of multidimensional Schur multipliers as elements of the extended Haagerup tensor product $L^{\infty}\left(X_{1}\right) \otimes_{e h} \ldots \otimes_{e h} L^{\infty}\left(X_{n}\right)$ (Theorem 3.4). This generalises the characterisations of Grothendieck and Peller in the case $n=2$. We show that the integral projective tensor product consists of multipliers and, therefore, $L^{\infty}\left(X_{1}\right) \hat{\otimes}_{i} \ldots \hat{\otimes}_{i} L^{\infty}\left(X_{n}\right) \subset L^{\infty}\left(X_{1}\right) \otimes_{e h} \ldots \otimes_{e h} L^{\infty}\left(X_{n}\right)$. The converse inclusion is true in the case $n=2$ [24] but remains an open problem for $n>2$.

In Section 4 we consider a non-commutative version of multidimensional multipliers following the Kissin-Shulman approach in the two-dimensional case. We replace the functions $\psi$ by elements of the minimal tensor product $\mathcal{A}_{1} \otimes \ldots \otimes \mathcal{A}_{n}$ of some given $C^{*}$-algebras $\mathcal{A}_{1}, \ldots, \mathcal{A}_{n}$ and the measure $\mu_{i}$ by a representation $\pi_{i}$ of $\mathcal{A}_{i}$. We thus obtain a class of operator $\left(\pi_{1}, \ldots, \pi_{n}\right)$-multipliers. If each $\mathcal{A}_{i}$ is a commutative $C^{*}$-algebra, say $\mathcal{A}_{i}=C_{0}\left(X_{i}\right)$ for some locally compact Hausdorff space $X_{i}$, and $\pi_{i}(f)$ is the operator of multiplication by $f \in C_{0}(X)$ acting on $L^{2}\left(X_{i}, \mu_{i}\right)$, then $\psi$ is a $\left(\pi_{1}, \ldots, \pi_{n}\right)$-multiplier if and only if $\psi$ is a Schur multiplier with respect to $\mu_{1}, \ldots, \mu_{n}$ (Proposition 4.6). As in the two-dimensional case, we show that the 
set of $\left(\pi_{1}, \ldots, \pi_{n}\right)$-multipliers does not change if we replace each $\pi_{i}$ by an approximately equivalent representation (Theorem 5.1). A consequence of this result is the fact that the class of continuous (multidimensional) Schur multipliers depends only on the supports of the measures $\mu_{i}$.

In Section 6] we study universal mutlipliers, that is, the elements of $\mathcal{A}_{1} \otimes \ldots \otimes \mathcal{A}_{n}$ which are $\left(\pi_{1}, \ldots, \pi_{n}\right)$-multipliers for all representations $\pi_{i}$ of $\mathcal{A}_{i}, i=1, \ldots, n$. We characterise such multipliers as the elements of a certain weak completion of the algebraic tensor product $\mathcal{A}_{1} \odot \ldots \odot \mathcal{A}_{n}$ (Theorem 6.6). In the case where the $C^{*}$-algebras are commutative and $n=2$ this was proved in 21]; the case of arbitrary $C^{*}$-algebras was left as a conjecture. Our result may be thought of as a non-commutative and multidimensional version of Grothendieck's and Peller's characterisations of Schur multipliers. A key ingredient in the proof is the observation that a universal multiplier determines a completely bounded multilinear modular map from the Cartesian product of the $C^{*}$-algebras of compact operators into the $C^{*}$-algebra of compact operators which allows us to use a result by Christensen and Sinclair 9] providing a description of all such mappings.

\section{Preliminaries}

In this section we collect some preliminary notions and results which will be needed in the sequel.

Let $H$ be a Hilbert space. The dual space $H^{\mathrm{d}}$ of $H$ is a Hilbert space and there exists an anti-isometry $\partial: H \rightarrow H^{\mathrm{d}}$ given by $\partial(x)(y)=(y, x), x, y \in H$. We set $x^{\mathrm{d}}=\partial(x)$.

If $H$ and $K$ are Hilbert spaces, we let $\mathcal{B}(H, K)$ be the space of all bounded linear operators from $H$ into $K$, and $\|\cdot\|_{\text {op }}$ be the usual operator norm on $\mathcal{B}(H, K)$. We let $\mathcal{K}(H, K)$ be the subspace of all compact operators, and $\mathcal{C}_{2}(H, K)$ be the subspace of all Hilbert-Schmidt operators, from $H$ into $K$. For each $T \in \mathcal{C}_{2}(H, K)$, we denote by $\|T\|_{2}$ the Hilbert-Schmidt norm of $T$. The space $\mathcal{C}_{2}(H, K)$ is a Hilbert space with respect to the inner product $(T, S)=\operatorname{tr}\left(T S^{*}\right)$, where $S^{*}$ denotes the adjoint of the operator $S$. We let $\mathcal{B}(H)=\mathcal{B}(H, H), \mathcal{K}(H)=\mathcal{K}(H, H)$ and $\mathcal{C}_{2}(H)=\mathcal{C}_{2}(H, H)$.

If $T \in \mathcal{B}(H, K)$ we denote by $T^{\mathrm{d}} \in B\left(K^{\mathrm{d}}, H^{\mathrm{d}}\right)$ the conjugate of $T$. We have that $\left\|T^{\mathrm{d}}\right\|_{\text {op }}=\|T\|_{\text {op }}$ and $T^{\mathrm{d}} x^{\mathrm{d}}=\left(T^{*} x\right)^{\mathrm{d}}$, whenever $x \in K_{2}$. Another way of expressing the last identity is

$$
T^{\mathrm{d}}=\partial T^{*} \partial^{-1}
$$

We also have

$$
\left(T^{*}\right)^{\mathrm{d}}=\left(T^{\mathrm{d}}\right)^{*} \text { and }(\lambda T)^{\mathrm{d}}=\lambda T^{\mathrm{d}}, \quad \lambda \in \mathbb{C} .
$$

We let $H \otimes K$ be the Hilbert space tensor product of $H$ and $K$. There exists a unitary operator $\theta: H \otimes K \rightarrow \mathcal{C}_{2}\left(H^{\mathrm{d}}, K\right)$ given on elementary tensors $x \otimes y \in H \otimes K$ by

$$
\theta(x \otimes y)\left(z^{\mathrm{d}}\right)=(x, z) y, \quad z^{\mathrm{d}} \in H^{\mathrm{d}} .
$$

If $A \in \mathcal{B}(H), B \in \mathcal{B}(K), x \in H$ and $y \in K$, we have that $\theta((A \otimes B)(x \otimes y))=$ $B \theta(x \otimes y) A^{\mathrm{d}}$, and hence

$$
\theta((A \otimes B) \xi)=B \theta(\xi) A^{\mathrm{d}} \quad \text { for all } \xi \in H \otimes K .
$$


If $\varphi \in \mathcal{B}(H \otimes K)$, let $\sigma(\varphi) \in \mathcal{B}\left(\mathcal{C}_{2}\left(H^{\mathrm{d}}, K\right)\right)$ be given by the formula

$$
\sigma(\varphi) \theta(\xi)=\theta(\varphi \xi), \quad \xi \in H \otimes K .
$$

Then $\sigma$ implements a unitary equivalence between $\mathcal{B}(H \otimes K)$ and $\mathcal{B}\left(\mathcal{C}_{2}\left(H^{\mathrm{d}}, K\right)\right)$. We will call an element $\varphi \in \mathcal{B}(H \otimes K)$ a concrete (operator) multiplier if there exists $C>0$ such that $\|\sigma(\varphi) T\|_{\text {op }} \leq C\|T\|_{\text {op }}$, for each $T \in \mathcal{C}_{2}\left(H^{\mathrm{d}}, K\right)$. Suppose that $H=l^{2}(X), K=l^{2}(Y)$ for some sets $X$ and $Y$ and $\varphi$ is the operator on $H \otimes K=\ell^{2}(X \times Y)$ of multiplication by a function $\phi \in \ell^{\infty}(X \times Y)$. The concrete operator multipliers of this form are precisely the classical Schur multipliers on $X \times Y$ (see e.g. 26]).

Let $\mathcal{A}$ and $\mathcal{B}$ be $C^{*}$-algebras. We denote by $\mathcal{A} \otimes \mathcal{B}$ the minimal tensor product of $\mathcal{A}$ and $\mathcal{B}$. Let $\pi: \mathcal{A} \rightarrow \mathcal{B}(H)$ (resp. $\rho: \mathcal{B} \rightarrow \mathcal{B}(K)$ ) be a representation of $\mathcal{A}$ (resp. $\mathcal{B})$. Then $\pi \otimes \rho: \mathcal{A} \otimes \mathcal{B} \rightarrow \mathcal{B}(H \otimes K)$, given on elementary tensors by $(\pi \otimes \rho)(a \otimes b)=\pi(a) \otimes \rho(b)$, is a representation of $\mathcal{A} \otimes \mathcal{B}$. Let $\sigma_{\pi, \rho}=\sigma \circ(\pi \otimes \rho)$; clearly, $\sigma_{\pi, \rho}$ is a representation of $\mathcal{A} \otimes \mathcal{B}$ on $\mathcal{C}_{2}\left(H^{\mathrm{d}}, K\right)$, unitarily equivalent to $\pi \otimes \rho$. We moreover have

$$
\sigma_{\pi, \rho}(a \otimes b) T=\rho(b) T \pi(a)^{\mathrm{d}}, \quad a \in \mathcal{A}, b \in \mathcal{B}, T \in \mathcal{C}_{2}\left(H^{\mathrm{d}}, K\right) .
$$

An element $\varphi \in \mathcal{A} \otimes \mathcal{B}$ is called a $(\pi, \rho)$-multiplier 21] if there exists $C>0$ such that

$$
\left\|\sigma_{\pi, \rho}(\varphi) T\right\|_{\mathrm{op}} \leq C\|T\|_{\mathrm{op}}, \quad \text { for each } T \in \mathcal{C}_{2}\left(H^{\mathrm{d}}, K\right),
$$

in other words, if $(\pi \otimes \rho)(\varphi)$ is a concrete operator multiplier. The set of all $(\pi, \rho)$ multipliers in $\mathcal{A} \otimes \mathcal{B}$ is denoted by $\mathbf{M}_{\pi, \rho}(\mathcal{A}, \mathcal{B})$, and the smallest constant $C$ appearing in (6) is denoted by $\|\varphi\|_{\pi, \rho}$. If $\varphi$ is a $(\pi, \rho)$-multiplier for all representations $\pi$ of $\mathcal{A}$ and $\rho$ of $\mathcal{B}$, then $\varphi$ is called a universal multiplier. The set of all universal multipliers is denoted by $\mathbf{M}(\mathcal{A}, \mathcal{B})$; if $\varphi \in \mathbf{M}(\mathcal{A}, \mathcal{B})$ we let $\|\varphi\|_{\text {univ }}=\sup _{\pi, \rho}\|\varphi\|_{\pi, \rho}$. It is not difficult to see that in this case $\|\varphi\|_{\text {univ }}<\infty[21$.

We now recall some notions from Operator Space Theory. We refer the reader to [7, 14] and 27] for more details. An operator space $\mathcal{E}$ is a closed subspace of $\mathcal{B}(H, K)$, for some Hilbert spaces $H$ and $K$. If $n, m \in \mathbb{N}$, by $M_{n, m}(\mathcal{E})$ we will denote the space of all $n$ by $m$ matrices with entries in $\mathcal{E}$ and let $M_{n}(\mathcal{E})=M_{n, n}(\mathcal{E})$. Note that $M_{n, m}(\mathcal{E})$ can be identified in a natural way with a subspace of $\mathcal{B}\left(H^{m}, K^{n}\right)$ and hence carries a natural operator norm. If $n=\infty$ or $m=\infty$, we will denote by $M_{n, m}(\mathcal{E})$ the space of all (singly or doubly infinite) matrices with entries in $\mathcal{E}$ which represent a bounded linear operator between the corresponding amplifications of the Hilbert spaces and set $M_{\infty}(\mathcal{E})=M_{\infty, \infty}(\mathcal{E})$. We also write $M_{n, m}=M_{n, m}(\mathbb{C})$ and $M_{\infty}=M_{\infty, \infty}(\mathbb{C})$. If $a=\left(a_{i j}\right) \in M_{n, m}(\mathcal{E})$, where $a_{i j} \in \mathcal{E}$, we let $a^{\mathrm{d}}=\left(a_{i j}^{\mathrm{d}}\right)$; thus $a^{\mathrm{d}} \in \mathcal{B}\left(K^{\mathrm{d}, m}, H^{\mathrm{d}, n}\right)$. We also let $a^{\mathrm{t}}=\left(a_{j i}\right) \in M_{m, n}(\mathcal{E})$; thus $a^{\mathrm{t}} \in \mathcal{B}\left(H^{n}, K^{m}\right)$. We have $\left\|a^{\mathrm{d}}\right\|_{\mathrm{op}}=\left\|a^{\mathrm{t}}\right\|_{\text {op }}$ and $\left\|a^{\mathrm{d}, \mathrm{t}}\right\|_{\mathrm{op}}=\|a\|_{\text {op }}$. The opposite operator space $\mathcal{E}^{o}$ of the operator space $\mathcal{E}$ is defined as follows: if $\mathcal{E} \subseteq \mathcal{B}(H, K)$, then $\mathcal{E}^{o}=\left\{x^{\mathrm{d}}: x \in\right.$ $\mathcal{E}\} \subseteq \mathcal{B}\left(K^{\mathrm{d}}, H^{\mathrm{d}}\right)$.

If $\mathcal{E}$ and $\mathcal{F}$ are operator spaces, a linear map $\Phi: \mathcal{E} \rightarrow \mathcal{F}$ is called completely bounded if the map $\Phi^{(k)}: M_{k}(\mathcal{E}) \rightarrow M_{k}(\mathcal{F})$, given by $\Phi^{(k)}\left(\left(a_{i j}\right)\right)=\left(\Phi\left(a_{i j}\right)\right)$, is bounded for each $k \in \mathbb{N}$ and $\|\Phi\|_{\mathrm{cb}} \stackrel{\text { def }}{=} \sup _{k}\left\|\Phi^{(k)}\right\|<\infty$.

Let $\mathcal{E}, \mathcal{E}_{1}, \ldots, \mathcal{E}_{n}$ be operator spaces. We denote by $\mathcal{E}_{1} \odot \cdots \odot \mathcal{E}_{n}$ the algebraic tensor product of $\mathcal{E}_{1}, \ldots, \mathcal{E}_{n}$. Let $a_{k}=\left(a_{i j}^{k}\right) \in M_{m_{k}, m_{k+1}}\left(\mathcal{E}_{k}\right), k=1, \ldots, n$. We 
denote by

$$
a^{1} \odot \cdots \odot a^{n} \in M_{m_{1}, m_{n+1}}\left(\mathcal{E}_{1} \odot \cdots \odot \mathcal{E}_{n}\right)
$$

the matrix whose $(i, j)$-entry is

$$
\sum_{i_{2}, \ldots, i_{n}} a_{i, i_{2}}^{1} \otimes a_{i_{2}, i_{3}}^{2} \otimes \cdots \otimes a_{i_{n}, j}^{n} .
$$

Let $\Phi: \mathcal{E}_{1} \times \cdots \times \mathcal{E}_{n} \rightarrow \mathcal{E}$ be a multilinear map and

$$
\Phi^{(m)}: M_{m}\left(\mathcal{E}_{1}\right) \times M_{m}\left(\mathcal{E}_{2}\right) \times \cdots \times M_{m}\left(\mathcal{E}_{n}\right) \rightarrow M_{m}(\mathcal{E})
$$

be the multilinear map given by

$$
\Phi^{(m)}\left(a^{1}, \ldots, a^{n}\right)_{i j}=\sum_{i_{2}, \ldots, i_{n}} \Phi\left(a_{i, i_{2}}^{1}, a_{i_{2}, i_{3}}^{2}, \ldots, a_{i_{n}, j}^{n}\right),
$$

where $a^{k}=\left(a_{i j}^{k}\right) \in M_{m}\left(\mathcal{E}_{k}\right), 1 \leq i, j \leq m$. The map $\Phi$ is called completely bounded if there exists $C>0$ such that for all $m \in \mathbb{N}$ and all elements $a^{k} \in M_{m}\left(\mathcal{E}_{k}\right)$, $k=1, \ldots, n$, we have

$$
\left\|\Phi^{(m)}\left(a^{1}, \ldots, a^{n}\right)\right\| \leq C\left\|a^{1}\right\| \ldots\left\|a^{n}\right\| .
$$

Every completely bounded multilinear map $\Phi: \mathcal{E}_{1} \times \cdots \times \mathcal{E}_{n} \rightarrow \mathcal{E}$ gives rise to a completely bounded linear map from the Haagerup tensor product $\mathcal{E}_{1} \otimes_{\mathrm{h}} \cdots \otimes_{\mathrm{h}} \mathcal{E}_{n}$ into $\mathcal{E}$. For details on the Haagerup tensor product we refer the reader to 14 .

If $R_{1}, \ldots, R_{n+1}$ are rings, $M_{i}$ is an $R_{i}$-left and $R_{i+1}$-right module for each $i=$ $1, \ldots, n$, and $M$ is an $\left(R_{1}, R_{n+1}\right)$-module, then a multilinear map $\Phi: M_{1} \times \cdots \times$ $M_{n} \rightarrow M$ will be called $\left(R_{1}, \ldots, R_{n+1}\right)$-modular (or simply modular if $R_{1}, \ldots, R_{n+1}$ are clear from the context) if

$$
\Phi\left(a_{1} m_{1} a_{2}, m_{2} a_{3}, \ldots, m_{n} a_{n+1}\right)=a_{1} \Phi\left(m_{1}, a_{2} m_{2}, a_{3} m_{3}, \ldots, a_{n} m_{n}\right) a_{n+1},
$$

for all $m_{i} \in M_{i}(i=1, \ldots, n)$ and $a_{j} \in R_{j}(j=1, \ldots, n+1)$. If $R_{i}=\mathcal{A}_{i}$ are $C^{*}$ algebras and $M_{i}=\mathcal{E}_{i}$ are operator spaces, we let $\mathcal{B}_{\mathcal{A}_{1}, \ldots, \mathcal{A}_{n+1}}\left(\mathcal{E}_{1}, \ldots, \mathcal{E}_{n} ; \mathcal{E}\right.$ ) (resp. $C B_{\mathcal{A}_{1}, \ldots, \mathcal{A}_{n+1}}\left(\mathcal{E}_{1}, \ldots, \mathcal{E}_{n} ; \mathcal{E}\right)$ ) denote the spaces of all bounded (resp. completely bounded) $\left(\mathcal{A}_{1}, \ldots, \mathcal{A}_{n+1}\right)$-modular maps from $\mathcal{E}_{1} \times \cdots \times \mathcal{E}_{n}$ into $\mathcal{E}$.

\section{Multidimensional Schur multipliers}

In this section, we define multidimensional Schur multipliers on the direct product of finitely many measure spaces. The main result of the section is Theorem 3.4. which characterises multidimensional Schur multipliers generalising the results of Peller 24] and Spronk 29].

Let $\left(X_{i}, \mu_{i}\right), i=1,2, \ldots, n$, be standard $\sigma$-finite measure spaces. For notational convenience, integration with respect to $\mu_{i}$ will be denoted by $d x_{i}$. Direct products of the form $X_{i_{1}} \times \cdots \times X_{i_{k}}$ will be equipped with the corresponding product measure. We equip the space $L^{2}\left(X_{1} \times X_{2}\right)$ with an $\left(L^{\infty}\left(X_{1}\right), L^{\infty}\left(X_{2}\right)\right)$-module action by letting $(a \xi b)(x, y)=a(x) \xi(x, y) b(y)$. We will denote by $M_{a}$ the operator of multiplication by the essentially bounded function $a$ acting on the corresponding $L^{2}$-space.

Theorem 3.1. A multilinear map

$$
S: L^{2}\left(X_{1} \times X_{2}\right) \times L^{2}\left(X_{2} \times X_{3}\right) \times \cdots \times L^{2}\left(X_{n-1} \times X_{n}\right) \rightarrow L^{2}\left(X_{1} \times X_{n}\right)
$$


is a bounded modular map if and only if there exists $\varphi \in L^{\infty}\left(X_{1} \times \cdots \times X_{n}\right)$ such that $S=S_{\varphi}$, where $S_{\varphi}\left(f_{1}, \ldots, f_{n-1}\right)\left(x_{1}, x_{n}\right)$ is defined as

$$
\int_{X_{2} \times \cdots \times X_{n-1}} \varphi\left(x_{1}, \ldots, x_{n}\right) f_{1}\left(x_{1}, x_{2}\right) f_{2}\left(x_{2}, x_{3}\right) \ldots f_{n-1}\left(x_{n-1}, x_{n}\right) d x_{2} \ldots d x_{n-1} .
$$

Moreover, $\left\|S_{\varphi}\right\|=\|\varphi\|_{\infty}$.

Proof. We first show that for each $\varphi$, the map $S_{\varphi}$ is a bounded modular map with norm not exceeding $\|\varphi\|_{\infty}$. For simplicity, we will assume in this part of the proof that $n=3$. Fix $\varphi, f_{1}$ and $f_{2}$. We have

$$
\begin{aligned}
\| S_{\varphi}( & \left.f_{1}, f_{2}\right) \|_{2}^{2} \leq \int_{X_{1} \times X_{3}}\left(\int\left|\varphi\left(x_{1}, x_{2}, x_{3}\right) f_{1}\left(x_{1}, x_{2}\right) f_{2}\left(x_{2}, x_{3}\right)\right| d x_{2}\right)^{2} d x_{1} d x_{3} \\
\leq & \|\varphi\|_{\infty}^{2} \int_{X_{1} \times X_{3}}\left(\int\left|f_{1}\left(x_{1}, x_{2}\right) f_{2}\left(x_{2}, x_{3}\right)\right| d x_{2}\right)^{2} d x_{1} d x_{3} \\
& \leq\|\varphi\|_{\infty}^{2} \int_{X_{1} \times X_{3}}\left(\int\left|f_{1}\left(x_{1}, x_{2}\right)\right|^{2} d x_{2}\right)\left(\int\left|f_{2}\left(x_{2}, x_{2}\right)\right|^{2} d x_{2}\right) d x_{1} d x_{3} \\
& =\|\varphi\|_{\infty}^{2}\left\|f_{1}\right\|_{2}^{2}\left\|f_{2}\right\|_{2}^{2} .
\end{aligned}
$$

Thus, $\varphi$ is bounded with $\left\|S_{\varphi}\right\| \leq\|\varphi\|_{\infty}$; the modularity of $S_{\varphi}$ is obvious.

Conversely, let

$$
S: L^{2}\left(X_{1} \times X_{2}\right) \times L^{2}\left(X_{2} \times X_{3}\right) \times \cdots \times L^{2}\left(X_{n-1} \times X_{n}\right) \rightarrow L^{2}\left(X_{1} \times X_{n}\right)
$$

be a bounded modular map. We first assume that the measures $\mu_{i}$ are finite. Write $K_{1}=L^{2}\left(X_{1} \times X_{n}\right)$ and let

$$
S_{1}: L^{2}\left(X_{2}\right) \times L^{2}\left(X_{2}\right) \times L^{2}\left(X_{3}\right) \times L^{2}\left(X_{3}\right) \times \cdots \times L^{2}\left(X_{n-1}\right) \times L^{2}\left(X_{n-1}\right) \rightarrow K_{1}
$$

be given by

$$
S_{1}\left(\xi_{2}, \eta_{2}, \xi_{3}, \eta_{3}, \ldots, \xi_{n-1}, \eta_{n-1}\right)=S\left(1 \otimes \xi_{2}, \eta_{2} \otimes \xi_{3}, \ldots, \eta_{n-1} \otimes 1\right)
$$

(here and in the sequel we denote by 1 the constant function taking value one). The fact that $S$ is modular implies that

$$
S_{1}\left(\xi_{2} a_{2}, \eta_{2}, \xi_{3} a_{3}, \ldots, \xi_{n-1} a_{n-1}, \eta_{n-1}\right)=S_{1}\left(\xi_{2}, a_{2} \eta_{2}, \xi_{3}, \ldots, a_{n-1} \eta_{n-1}\right),
$$

whenever $a_{i} \in L^{\infty}\left(X_{i}\right), i=2, \ldots, n-1$. For fixed $\xi_{3}, \eta_{3}, \ldots, \xi_{n-1}, \eta_{n-1}$, let $S_{2}$ : $L^{2}\left(X_{2}\right) \times L^{2}\left(X_{2}\right) \rightarrow K_{1}$ be given by

$$
S_{2}\left(\xi_{2}, \eta_{2}\right)=S_{1}\left(\xi_{2}, \eta_{2}, \xi_{3}, \eta_{3}, \ldots, \xi_{n-1}, \eta_{n-1}\right) .
$$

For $h \in K_{1}$, let $S_{2}^{h}: L^{2}\left(X_{2}\right) \times L^{2}\left(X_{2}\right) \rightarrow \mathbb{C}$ be defined by $S_{2}^{h}\left(\xi_{2}, \eta_{2}\right)=\left(S_{2}\left(\xi_{2}, \eta_{2}\right), h\right)$. Clearly,

$$
\left|S_{2}^{h}\left(\xi_{2}, \eta_{2}\right)\right| \leq\|h\|\|S\| \prod_{i=2}^{n-1}\left\|\xi_{i}\right\|\left\|\eta_{i}\right\| .
$$

Hence there exists a bounded operator $T_{2}^{h}: L^{2}\left(X_{2}\right) \rightarrow L^{2}\left(X_{2}\right)$ such that $S_{2}^{h}\left(\xi_{2}, \eta_{2}\right)$ $=\left(T_{2}^{h} \xi_{2}, \overline{\eta_{2}}\right)$, for all $\xi_{2}, \eta_{2} \in L^{2}\left(X_{2}\right)$ and $\left\|T_{2}^{h}\right\| \leq\|h\|\|S\| \prod_{i=3}^{n-1}\left\|\xi_{i}\right\|\left\|\eta_{i}\right\|$. For each $a \in L^{\infty}\left(X_{2}\right)$ and $\xi_{2}, \eta_{2} \in L^{2}\left(X_{2}\right)$, we have that

$$
\begin{aligned}
\left(T_{2}^{h} M_{a} \xi_{2}, \overline{\eta_{2}}\right) & =S_{2}^{h}\left(a \xi_{2}, \eta_{2}\right)=S_{2}^{h}\left(\xi_{2}, a \eta_{2}\right) \\
& =\left(T_{2}^{h} \xi_{2}, \overline{a \eta_{2}}\right)=\left(T_{2}^{h} \xi_{2}, M_{\bar{a}} \overline{\eta_{2}}\right)=\left(M_{a} T_{2}^{h} \xi_{2}, \overline{\eta_{2}}\right) .
\end{aligned}
$$


Thus, there exists $\varphi_{2}^{h} \in L^{\infty}\left(X_{2}\right)$ such that $T_{2}^{h}=M_{\varphi_{2}^{h}}$. Moreover,

$$
\left\|\varphi_{2}^{h}\right\|_{\infty} \leq\|h\|\|S\| \prod_{i=3}^{n-1}\left\|\xi_{i}\right\|\left\|\eta_{i}\right\|
$$

For each $f \in L^{1}\left(X_{2}\right)$, the functional on $K_{1}$ given by $h \rightarrow \int_{X_{2}} f\left(x_{2}\right) \varphi_{2}^{h}\left(x_{2}\right) d x_{2}$ is conjugate linear and bounded with norm not exceeding $\|f\|_{1}\|S\| \prod_{i=3}^{n-1}\left\|\xi_{i}\right\|\left\|\eta_{i}\right\|$. Hence, there exists $\Phi_{2}(f) \in K_{1}$ such that

$$
\left(\Phi_{2}(f), h\right)=\int_{X_{2}} f\left(x_{2}\right) \varphi_{2}^{h}\left(x_{2}\right) d x_{2},
$$

and $\left\|\Phi_{2}(f)\right\|_{K_{1}} \leq\|f\|_{1}\|S\| \prod_{i=3}^{n-1}\left\|\xi_{i}\right\|\left\|\eta_{i}\right\|$. Thus, the mapping $\Phi_{2}: L^{1}\left(X_{2}\right) \rightarrow K_{1}$ is bounded and $\left\|\Phi_{2}\right\| \leq\|S\| \prod_{i=3}^{n-1}\left\|\xi_{i}\right\|\left\|\eta_{i}\right\|$. Since Hilbert spaces possess the RadonNikodým property, the vector-valued Riesz Representation Theorem 11, Theorem 5 , p. 63] implies that there exists $\varphi_{2} \in L^{\infty}\left(X_{2}, K_{1}\right)\left(L^{\infty}\left(X_{2}, K_{1}\right)\right.$ being the space of essentially bounded $K_{1}$-valued measurable functions on $\left.X_{2}\right)$ such that

$$
\Phi_{2}(f)=\int_{X_{2}} f\left(x_{2}\right) \varphi_{2}\left(x_{2}\right) d x_{2},
$$

where the integral is in Bochner's sense. Moreover,

$$
\left\|\varphi_{2}\right\|_{L^{\infty}\left(X_{2}, K_{1}\right)}=\underset{x_{2} \in X_{2}}{\operatorname{ess} \sup _{2}}\left\|\varphi_{2}\left(x_{2}\right)\right\|_{K_{1}}=\left\|\Phi_{2}\right\| \leq\|S\| \prod_{i=3}^{n-1}\left\|\xi_{i}\right\|\left\|\eta_{i}\right\| .
$$

For $\xi_{2}, \eta_{2} \in L^{2}\left(X_{2}\right)$, we have that $\xi_{2} \overline{\eta_{2}} \in L^{1}\left(X_{2}\right)$ and hence

$$
\begin{aligned}
\left(S_{2}\left(\xi_{2}, \eta_{2}\right), h\right) & =\left(T_{2}^{h} \xi_{2}, \overline{\eta_{2}}\right)=\int_{X_{2}} \varphi_{2}^{h}\left(x_{2}\right) \xi_{2}\left(x_{2}\right) \eta_{2}\left(x_{2}\right) d x_{2} \\
& =\left(\int_{X_{2}} \varphi_{2}\left(x_{2}\right) \xi_{2}\left(x_{2}\right) \eta_{2}\left(x_{2}\right) d x_{2}, h\right) ;
\end{aligned}
$$

in other words,

$$
S_{2}\left(\xi_{2}, \eta_{2}\right)=\int_{X_{2}} \varphi_{2}\left(x_{2}\right) \xi_{2}\left(x_{2}\right) \eta_{2}\left(x_{2}\right) d x_{2},
$$

where the integral is in Bochner's sense.

We consider $\varphi_{2}$ as a function on $X_{1} \times X_{2} \times X_{n}$ by letting $\varphi_{2}\left(x_{1}, x_{2}, x_{n}\right)$ $=\varphi_{2}\left(x_{2}\right)\left(x_{1}, x_{n}\right)$. Note that $\varphi_{2}$ depends on $\xi_{3}, \eta_{3}, \ldots, \xi_{n-1}, \eta_{n-1}$; we denote this dependence by $\varphi_{2}=\varphi_{2, \xi_{3}, \eta_{3}, \ldots, \xi_{n-1}, \eta_{n-1}}$.

Let $K_{2}=L^{2}\left(X_{1} \times X_{2} \times X_{n}\right)$. We have

$$
\begin{aligned}
\left\|\varphi_{2}\right\|_{K_{2}} & =\int_{X_{2}} \int_{X_{1} \times X_{n}}\left|\varphi_{2}\left(x_{2}\right)\left(x_{1}, x_{n}\right)\right|^{2} d x_{1} d x_{n} d x_{2} \\
& =\int_{X_{2}}\left\|\varphi_{2}\left(x_{2}\right)\right\|_{K_{1}}^{2} d x_{2} \leq \mu_{2}\left(X_{2}\right)\left\|\varphi_{2}\right\|_{L^{\infty}\left(X_{2}, K_{1}\right)} .
\end{aligned}
$$

It follows that the mapping $S_{3}: L^{2}\left(X_{3}\right) \times L^{2}\left(X_{3}\right) \rightarrow K_{2}$, given by

$$
S_{3}\left(\xi_{3}, \eta_{3}\right)=\varphi_{2, \xi_{3}, \eta_{3}, \ldots, \xi_{n-1}, \eta_{n-1}},
$$

is well-defined and

$$
\left\|S_{3}\left(\xi_{3}, \eta_{3}\right)\right\|_{K_{2}} \leq \mu_{2}\left(X_{2}\right)\|S\| \prod_{i=3}^{n-1}\left\|\xi_{i}\right\|\left\|\eta_{i}\right\| .
$$


Hence, $S_{3}$ is bounded and $\left\|S_{3}\right\| \leq \mu_{2}\left(X_{2}\right)\|S\| \prod_{i=4}^{n-1}\left\|\xi_{i}\right\|\left\|\eta_{i}\right\|$. An argument similar to the above implies the existence of $\varphi_{3} \in L^{\infty}\left(X_{3}, K_{2}\right)$ with

$$
\left\|\varphi_{3}\right\|_{L^{\infty}\left(X_{3}, K_{2}\right)} \leq \mu_{2}\left(X_{2}\right)\|S\| \prod_{i=4}^{n-1}\left\|\xi_{i}\right\|\left\|\eta_{i}\right\|,
$$

such that

$$
S_{3}\left(\xi_{3}, \eta_{3}\right)=\int_{X_{3}} \varphi_{3}\left(x_{3}\right) \xi_{3}\left(x_{3}\right) \eta_{3}\left(x_{3}\right) d x_{3},
$$

where the integral is in Bochner's sense. We may consider $\varphi_{3}$ as a function on $X_{1} \times X_{2} \times X_{3} \times X_{n}$ by letting $\varphi_{3}\left(x_{1}, x_{2}, x_{3}, x_{n}\right)=\varphi_{3}\left(x_{3}\right)\left(x_{1}, x_{2}, x_{n}\right)$. We express the dependence of $\varphi_{3}$ on $\xi_{4}, \ldots, \eta_{n-1}$ by writing $\varphi_{3}=\varphi_{3, \xi_{4}, \ldots, \eta_{n-1}}$. We have that

$$
\begin{aligned}
& S_{1}\left(\xi_{2}, \eta_{2}, \ldots, \xi_{n-1}, \eta_{n-1}\right) \\
& =\int_{X_{2}} \int_{X_{3}} \varphi_{3, \xi_{4}, \ldots, \eta_{n-1}}\left(x_{1}, x_{2}, x_{3}, x_{n}\right) \xi_{2}\left(x_{2}\right) \eta_{2}\left(x_{2}\right) \xi_{3}\left(x_{3}\right) \eta_{3}\left(x_{3}\right) d x_{3} d x_{2},
\end{aligned}
$$

where both integrals are in Bochner's sense.

Continuing inductively, we obtain $\varphi \in L^{\infty}\left(X_{n-1}, K_{n-2}\right)$, where $K_{n-2}=$ $L^{2}\left(X_{1} \times \cdots \times X_{n-2} \times X_{n}\right)$, such that

$$
\begin{aligned}
& S_{1}\left(\xi_{2}, \eta_{2}, \ldots, \xi_{n-1}, \eta_{n-1}\right) \\
& =\int_{X_{2}} \ldots \int_{X_{n-1}} \varphi\left(x_{1}, \ldots, x_{n}\right) \xi_{2} \eta_{2} \ldots \xi_{n-1} \eta_{n-1} d x_{n-1} \ldots d x_{2}
\end{aligned}
$$

where the integrals are understood in Bochner's sense and $\varphi$ is viewed as a function on $X_{1} \times \cdots \times X_{n}$ by letting

$$
\varphi\left(x_{1}, \ldots, x_{n}\right)=\varphi\left(x_{n-1}\right)\left(x_{1}, \ldots, x_{n-2}, x_{n}\right) .
$$

It is easy to see that if $\psi \in L^{1}\left(Y, L^{2}(Z)\right)$, where $Y$ and $Z$ are finite measure spaces, then $\int_{Y \times Z}|\psi(y)(z)| d y d z$ is finite and $\left(\int_{Y} \psi(y) d y\right)(z)=\int_{Y} \psi(y)(z) d y$, for almost all $z \in Z$ (the first integral is in Bochner's sense, while the second one is a Lebesgue integral with respect to the variable $y$ ). It now follows that the last equality holds when the integrals are interpreted in the sense of Lebesgue.

The modularity of $S$ implies

$$
\begin{aligned}
& S\left(a \otimes \xi_{2}, \eta_{2} \otimes \xi_{3}, \ldots, \eta_{n-1} \otimes b\right) \\
& =\int_{X_{2}} \int_{X_{3}} \ldots \int_{X_{n-1}} \varphi\left(x_{1}, \ldots, x_{n}\right) a \xi_{2} \eta_{2} \ldots \xi_{n-1} \eta_{n-1} b d x_{n-1} \ldots d x_{2},
\end{aligned}
$$

for all $a \in L^{\infty}\left(X_{1}\right), b \in L^{\infty}\left(X_{n}\right)$ and $\xi_{i}, \eta_{i} \in L^{2}\left(X_{i}\right), i=2, \ldots, n-1$. Letting $a=\chi_{\alpha_{1}}, b=\chi_{\alpha_{n}}$ and $\xi_{i}=\eta_{i}=\chi_{\alpha_{i}}, i=2, \ldots, n-1$, the boundedness of $S$ implies

$$
\int_{\alpha_{1} \times \cdots \times \alpha_{n}}\left|\varphi\left(x_{1}, \ldots, x_{n}\right)\right| d x_{1} \ldots d x_{n} \leq\|S\| \mu_{1}\left(\alpha_{1}\right) \ldots \mu_{n}\left(\alpha_{n}\right) .
$$

It follows that the mapping

$$
f=\sum_{i=1}^{N} \lambda_{i} \chi_{\alpha_{1}^{i} \times \cdots \times \alpha_{n}^{i}} \longrightarrow \int_{X_{1} \times \cdots \times X_{n}} \varphi f
$$

where $\left\{\alpha_{1}^{i} \times \cdots \times \alpha_{n}^{i}\right\}$ is a finite family of disjoint Borel rectangles, is a linear functional on a dense subspace of $L^{1}\left(X_{1} \times \cdots \times X_{n}\right)$ of norm not exceeding $\|S\|$. Therefore, $\varphi \in L^{\infty}\left(X_{1} \times \cdots \times X_{n}\right)$ and $\|\varphi\|_{\infty} \leq\|S\|$. 
We have that the mappings $S$ and $S_{\varphi}$ coincide on the tuples of the form $a \otimes$ $\xi_{2}, \eta_{2} \otimes \xi_{3}, \ldots, \eta_{n-1} \otimes b$; by linearity and continuity, they are equal. By the first part of the proof, $\|S\| \leq\|\varphi\|_{\infty}$ and hence $\|\varphi\|_{\infty}=\|S\|$.

Now relax the assumption on the finiteness of $\mu_{i}$, and let $X_{i}^{k}, k \in \mathbb{N}$, be a measurable subset of $X_{i}$ such that $\mu_{i}\left(X_{i}^{k}\right)<\infty, X_{i}^{k} \subseteq X_{i}^{k+1}$ and $X_{i}=\bigcup_{k=1}^{\infty} X_{i}^{k}$, $i=1, \ldots, n$. For each $k \in \mathbb{N}$, let

$$
S_{k}: L^{2}\left(X_{1}^{k} \times X_{2}^{k}\right) \times L^{2}\left(X_{2}^{k} \times X_{3}^{k}\right) \times \cdots \times L^{2}\left(X_{n-1}^{k} \times X_{n}^{k}\right) \rightarrow L^{2}\left(X_{1}^{k} \times X_{n}^{k}\right)
$$

be the map given by $S_{k}\left(f_{1}, \ldots, f_{n-1}\right)=S\left(\tilde{f}_{1}, \ldots, \tilde{f}_{n-1}\right)$, where $\tilde{f}_{i}$ coincides with $f_{i}$ on $X_{i}^{k}$ and is equal to zero on the complement of $X_{i}^{k}$. Since

$$
\begin{aligned}
S_{k}\left(f_{1}, \ldots, f_{n-1}\right) & =S\left(\chi_{X_{1}^{k}} \tilde{f}_{1}, \ldots, \tilde{f}_{n-1} \chi_{X_{n}^{k}}\right) \\
& =\chi_{X_{1}^{k}} S\left(\tilde{f}_{1}, \ldots, \tilde{f}_{n-1}\right) \chi_{X_{n}^{k}},
\end{aligned}
$$

the map $S_{k}$ is well-defined and $\left\|S_{k}\right\| \leq\|S\|$. Since $S_{k}$ is obviously $\left(L^{\infty}\left(X_{n}^{k}\right), \ldots\right.$, $\left.L^{\infty}\left(X_{1}^{k}\right)\right)$-modular, the above paragraphs imply that there exists $\varphi_{k} \in L^{\infty}\left(X_{1}^{k} \times\right.$ $\left.\cdots \times X_{n}^{k}\right)$ such that $S_{k}=S_{\varphi_{k}}$, for each $k \in \mathbb{N}$. The space $L^{2}\left(X_{i}^{k} \times X_{i+1}^{k}\right)$ can be considered as a subspace of $L^{2}\left(X_{i}^{k+1} \times X_{i+1}^{k+1}\right)$ in a natural way. We have that the restriction of $S_{k+1}$ to $L^{2}\left(X_{1}^{k} \times X_{2}^{k}\right) \times L^{2}\left(X_{2}^{k} \times X_{3}^{k}\right) \times \ldots \times L^{2}\left(X_{n-1}^{k} \times X_{n}^{k}\right)$ coincides with $S_{k}$. This implies that the restriction of $\varphi_{k+1}$ to $X_{1}^{k} \times \cdots \times X_{n}^{k}$ coincides (almost everywhere) with $\varphi_{k}$. Hence, there exists a function $\varphi$ defined on $X_{1} \times \cdots \times X_{n}$ which coincides with $\varphi_{k}$ on $X_{1}^{k} \times \cdots \times X_{n}^{k}$, for each $k \in \mathbb{N}$. Since $\left\|\varphi_{k}\right\|_{\infty}=\left\|S_{k}\right\| \leq\|S\|$, we have that $\|\varphi\|_{\infty} \leq\|S\|$. We have that $S$ and $S_{\varphi}$ coincide on the union of $L^{2}\left(X_{1}^{k} \times X_{2}^{k}\right) \times L^{2}\left(X_{2}^{k} \times X_{3}^{k}\right) \times \ldots \times L^{2}\left(X_{n-1}^{k} \times X_{n}^{k}\right), k \in \mathbb{N}$, which is a dense subset of $L^{2}\left(X_{1} \times X_{2}\right) \times L^{2}\left(X_{2} \times X_{3}\right) \times \ldots \times L^{2}\left(X_{n-1} \times X_{n}\right)$. It follows that $S=S_{\varphi}$, and by the first part of the proof, $\|S\|=\|\varphi\|_{\infty}$.

Let

$$
\Gamma\left(X_{1}, \ldots, X_{n}\right)=L^{2}\left(X_{1} \times X_{2}\right) \odot \cdots \odot L^{2}\left(X_{n-1} \times X_{n}\right) .
$$

We identify the elements of $\Gamma\left(X_{1}, \ldots, X_{n}\right)$ with functions on

$$
X_{1} \times X_{2} \times X_{2} \times \cdots \times X_{n-1} \times X_{n-1} \times X_{n}
$$

in the obvious fashion. We equip $\Gamma\left(X_{1}, \ldots, X_{n}\right)$ with two norms; one is the projective norm $\|\cdot\|_{2, \wedge}$, where each of the $L^{2}$-spaces is equipped with its $L^{2}$-norm, and the other is the Haagerup tensor norm $\|\cdot\|_{\mathrm{h}}$, where the $L^{2}$-spaces are given their opposite operator space structure arising from the identification of $L^{2}(X \times Y)$ with the class of Hilbert-Schmidt operators from $L^{2}(X)$ into $L^{2}(Y)$ given by

$$
\left(T_{f} \xi\right)(y)=\int_{X} f(x, y) \xi(x) d x, \quad f \in L^{2}(X \times Y), \xi \in L^{2}(X) .
$$

For each $\varphi \in L^{\infty}\left(X_{1} \times \cdots \times X_{n}\right)$, we consider the linearisation of the map $S_{\varphi}$ from Theorem 3.1 to a map defined on $\Gamma\left(X_{1}, \ldots, X_{n}\right)$ and taking values in $L^{2}\left(X_{1} \times X_{n}\right)$ and we denote it in the same way. Thus, if $f_{1} \otimes \cdots \otimes f_{n-1}$ is in $\Gamma\left(X_{1}, \ldots, X_{n}\right)$, then $S_{\varphi}\left(f_{1} \otimes \cdots \otimes f_{n-1}\right)\left(x_{1}, x_{n}\right)$ is equal to

$$
\int_{X_{2} \times \cdots \times X_{n-1}} \varphi\left(x_{1}, \ldots, x_{n}\right) f_{1}\left(x_{1}, x_{2}\right) f_{2}\left(x_{2}, x_{3}\right) \ldots f_{n-1}\left(x_{n-1}, x_{n}\right) d x_{2} \ldots d x_{n-1} .
$$

By Theorem 3.1, $S_{\varphi}$ is bounded and $\left\|S_{\varphi}\right\|=\|\varphi\|_{\infty}$. Hence it extends to a bounded map from $\left(\Gamma\left(X_{1}, \ldots, X_{n}\right),\|\cdot\|_{2, \wedge}\right)$ into $\left(L^{2}\left(X_{1} \times X_{n}\right),\|\cdot\|_{2}\right)$. 
Definition 3.2. Let $\varphi \in L^{\infty}\left(X_{1} \times \cdots \times X_{n}\right)$. We say that $\varphi$ is a Schur multiplier (relative to the measure spaces $\left.\left(X_{1}, \mu_{1}\right), \ldots,\left(X_{n}, \mu_{n}\right)\right)$ if there exists $C>0$ such that $\left\|S_{\varphi}(\Phi)\right\|_{\text {op }} \leq C\|\Phi\|_{\mathrm{h}}$, for all $\Phi \in \Gamma\left(X_{1}, \ldots, X_{n}\right)$. The smallest constant $C$ with this property will be denoted by $\|\varphi\|_{\mathrm{m}}$.

We will present next a characterisation of the $n$-dimensional Schur multipliers which generalises Grothendieck's and Peller's characterisations. We will need the following generalisation of a result of Smith [28].

Lemma 3.3. Let $\mathcal{E}_{i} \subseteq B\left(H_{i}, H_{i+1}\right), i=1, \ldots, n$ be spaces of operators and $\mathcal{C} \subseteq$ $B\left(H_{1}\right), \mathcal{D} \subseteq B\left(H_{n+1}\right)$ be $C^{*}$-algebras with cyclic vectors. Assume that $\mathcal{E}_{1}$ is a right $\mathcal{C}$-module and $\mathcal{E}_{n}$ is a left $\mathcal{D}$-module. Let $\phi: \mathcal{E}_{n} \times \cdots \times \mathcal{E}_{1} \rightarrow B\left(H_{1}, H_{n+1}\right)$ be a multilinear $(\mathcal{D}, \mathcal{C})$-module map (that is, $\phi(d y, \ldots, x c)=d \phi(y, \ldots, x) c$, whenever $x \in \mathcal{E}_{1}, y \in \mathcal{E}_{n}, c \in \mathcal{C}$ and $d \in \mathcal{D}$ ) such that the corresponding linear map from $\mathcal{E}_{n} \odot \cdots \odot \mathcal{E}_{1}$ into $B\left(H_{1}, H_{n+1}\right)$ is bounded in the Haagerup norm. Then $\phi$ is a completely bounded multilinear map.

Proof. The proof is a straightforward generalisation of the argument given by Smith 28. We will denote by $\tilde{\phi}$ the linear map from $\mathcal{E}_{n} \odot \cdots \odot \mathcal{E}_{1}$ into $\mathcal{B}\left(H_{1}, H_{n+1}\right)$ defined by $\tilde{\phi}\left(a_{n} \otimes \cdots \otimes a_{1}\right)=\phi\left(a_{n}, \ldots, a_{1}\right)$. By the assumption of the lemma, it is bounded in the Haagerup norm $\|\cdot\|_{\mathrm{h}}$. Assume that $\|\tilde{\phi}\|=1$. We will show that $\|\tilde{\phi}\|_{\mathrm{cb}}=1$. Suppose, to the contrary, that $\|\tilde{\phi}\|_{\mathrm{cb}}>1$. Then there exists $m \in \mathbb{N}$, matrices $x^{i}=\left(x_{k j}^{i}\right) \in M_{m}\left(\mathcal{E}_{i}\right), i=1, \ldots, n$ and column vectors $\xi_{0}=\left(\xi_{1}, \ldots, \xi_{m}\right) \in H_{1}^{m}$ and $\eta_{0}=\left(\eta_{1}, \ldots, \eta_{m}\right) \in H_{n+1}^{m}$ such that $\left\|\xi_{0}\right\|<1,\left\|\eta_{0}\right\|<1$, all $\left\|x^{i}\right\|<1$ and

$$
\left|\left(\phi^{(m)}\left(x^{n}, x^{n-1}, \ldots, x^{1}\right) \xi_{0}, \eta_{0}\right)\right|>1 .
$$

If $\xi$ and $\eta$ are cyclic vectors for $\mathcal{C}$ and $\mathcal{D}$, respectively, we may moreover assume that $\xi_{i}=a_{i} \xi$ and $\eta_{j}=b_{j} \eta$, for some $a_{i} \in \mathcal{C}$ and $b_{j} \in \mathcal{D}$, where $i, j=1, \ldots, m$. Let $a=\sum_{i=1}^{m} a_{i}^{*} a_{i}$ and $b=\sum_{j=1}^{m} b_{j}^{*} b_{j}$. Assume first that $a$ and $b$ are invertible, and let $c_{i}=a_{i} a^{-1 / 2}, d_{j}=b_{j} b^{-1 / 2}, \tilde{\xi}=a^{1 / 2} \xi$ and $\tilde{\eta}=b^{1 / 2} \eta$. Then $\xi_{i}=c_{i} \tilde{\xi}$ and $\eta_{j}=d_{j} \tilde{\eta}$. Taking into account (9), the left-hand side of (11) becomes

$$
\begin{aligned}
& \left|\sum_{i, j=1}^{m}\left(\phi^{(m)}\left(x^{n}, x^{n-1}, \ldots, x^{1}\right)_{j i} c_{i} \tilde{\xi}, d_{j} \tilde{\eta}\right)\right| \\
= & \left|\sum_{k_{1}, \ldots, k_{n-1}=1}^{m} \sum_{i, j=1}^{m}\left(\phi\left(d_{j}^{*} x_{j k_{n-1}}^{n}, x_{k_{n-1} k_{n-2}}^{n-1}, \ldots, x_{k_{1} i}^{1} c_{i}\right) \tilde{\xi}, \tilde{\eta}\right)\right| \\
= & \left|\sum_{k_{1}, \ldots, k_{n-1}=1}^{m}\left(\phi\left(\sum_{j=1}^{m} d_{j}^{*} x_{j k_{n-1}}^{n}, x_{k_{n-1} k_{n-2}}^{n-1}, \ldots, \sum_{i=1}^{m} x_{k_{1}, i}^{1} c_{i}\right) \tilde{\xi}, \tilde{\eta}\right)\right| \\
\leq & \left\|\sum_{k_{1}, \ldots, k_{n-1}=1}^{m} \phi\left(\sum_{j=1}^{m} d_{j}^{*} x_{j k_{n-1}}^{n}, x_{k_{n-1} k_{n-2}}^{n-1}, \ldots, \sum_{i=1}^{m} x_{k_{1}, i}^{1} c_{i}\right)\right\|\|\tilde{\xi}\|\|\tilde{\eta}\| .
\end{aligned}
$$

We have that

$$
\|\tilde{\xi}\|=\left(a^{1 / 2} \xi, a^{1 / 2} \xi\right)=(a \xi, \xi)=\sum_{k=1}^{n}\left\|a_{i} \xi\right\|^{2}=\sum_{k=1}^{n}\left\|\xi_{i}\right\|^{2}=\left\|\xi_{0}\right\| \leq 1,
$$


and similarly $\|\tilde{\eta}\| \leq 1$. Set $d^{*}=\left(d_{j}^{*}\right) \in M_{1, m}(\mathcal{D}), c=\left(c_{i}\right) \in M_{m, 1}(\mathcal{C}), u=d^{*} x^{n} \in$ $M_{1, m}\left(\mathcal{E}_{n}\right)$ and $v=x^{1} c \in M_{m, 1}\left(\mathcal{E}_{1}\right)$. It follows from (7) and (8) that

$$
\begin{aligned}
& \left\|\sum_{k_{1}, \ldots, k_{n-1}=1}^{m} \phi\left(\sum_{j=1}^{m} d_{j}^{*} x_{j k_{n-1}}, x_{k_{n-1} k_{n-2}}^{n-1}, \ldots, \sum_{i=1}^{m} x_{k_{1}, i} c_{i}\right)\right\| \\
= & \left\|\sum_{k_{1}, \ldots, k_{n-1}=1}^{m} \phi\left(u_{k_{n-1}}, x_{k_{n-1} k_{n-2}}^{n-1}, \ldots, v_{k_{1}}\right)\right\| \\
= & \left\|\tilde{\phi}\left(\sum_{k_{1}, \ldots, k_{n-1}=1}^{m} u_{k_{n-1}} \otimes x_{k_{n-1} k_{n-2}}^{n-1} \otimes \cdots \otimes v_{k_{1}}\right)\right\| \\
\leq & \left\|\sum_{k_{1}, \ldots, k_{n-1}=1}^{m} u_{k_{n-1}} \otimes x_{k_{n-1} k_{n-2}}^{n-1} \otimes \cdots \otimes v_{k_{1}}\right\|_{\mathrm{h}} \\
= & \left\|u \odot x^{n-1} \odot \cdots \odot x^{2} \odot v\right\|_{\mathrm{h}} \\
\leq & \left\|d^{*}\right\|\left\|x^{n}\right\|\left\|x^{n-1}\right\| \ldots\left\|x^{2}\right\|\left\|x^{1}\right\|\|c\| .
\end{aligned}
$$

We have that

$$
\left\|d^{*}\right\|=\left\|\sum_{j=1}^{m} d_{j}^{*} d_{j}\right\|^{1 / 2}=\|I\|=1
$$

and, similarly, $\|c\|=1$. It follows from (12) and (13) that

$$
\left|\left(\phi^{(m)}\left(x^{n}, x^{n-1}, \ldots, x^{1}\right) \xi_{0}, \eta_{0}\right)\right| \leq 1,
$$

which contradicts (11).

In the case that $a$ or $b$ is not invertible, one can again follow [28] and, for each $i$, consider the matrix $\hat{x}^{i} \in M_{m+1}\left(\mathcal{E}_{i}\right)$ which has the matrix $x^{i}$ in its upper left corner and zeros in the last row and column. The vectors $\xi_{0}$ and $\eta_{0}$ are replaced with $\hat{\xi}_{0}=\left(\xi_{1}, \ldots, \xi_{m}, \xi_{m+1}\right)$ and $\hat{\eta}_{0}=\left(\eta_{1}, \ldots, \eta_{m}, \eta_{m+1}\right)$, where $\xi_{m+1}=\epsilon \xi$ and $\eta_{m+1}=\epsilon \eta$, respectively, for $\epsilon$ small enough so that the norms of these vectors remain less than one. Letting $a_{n+1}=b_{n+1}=\epsilon I$, we have that $a_{i} \xi=\xi_{i}$ and $b_{i} \eta=\eta_{i}$ for each $i=1, \ldots, m+1$. Finally,

$$
\left(\phi^{(m)}\left(x^{n}, x^{n-1}, \ldots, x^{1}\right) \xi_{0}, \eta_{0}\right)=\left(\phi^{(m+1)}\left(\hat{x}^{n}, \hat{x}^{n-1}, \ldots, \hat{x}^{1}\right) \hat{\xi}_{0}, \hat{\eta}_{0}\right)
$$

and the proof proceeds as before.

The main result of this section is the following.

Theorem 3.4. Let $\varphi \in L^{\infty}\left(X_{1} \times \cdots \times X_{n}\right)$. The following are equivalent:

(i) $\varphi$ is a Schur multiplier and $\|\varphi\|_{\mathrm{m}}<1$;

(ii) there exist essentially bounded functions $a_{1}: X_{1} \rightarrow M_{\infty, 1}, a_{n}: X_{n} \rightarrow M_{1, \infty}$ and $a_{i}: X_{i} \rightarrow M_{\infty}, i=2, \ldots, n-1$, such that, for almost all $x_{1}, \ldots, x_{n}$ we have

$$
\varphi\left(x_{1}, \ldots, x_{n}\right)=a_{n}\left(x_{n}\right) a_{n-1}\left(x_{n-1}\right) \ldots a_{1}\left(x_{1}\right) \text { and } \underset{x_{i} \in X_{i}}{\operatorname{ess} \sup _{i}} \prod_{i=1}^{n}\left\|a_{i}\left(x_{i}\right)\right\|<1 .
$$

Proof. (i) $\Rightarrow$ (ii) Let $\varphi \in L^{\infty}\left(X_{1} \times \cdots \times X_{n}\right)$ be a Schur multiplier with $\|\varphi\|_{\mathrm{m}}<1$. Then the map $S_{\varphi}$ induces a map, denoted in the same way, from $L^{2}\left(X_{1} \times X_{2}\right) \times \cdots \times$ 
$L^{2}\left(X_{n-1} \times X_{n}\right)$ into $L^{2}\left(X_{1} \times X_{n}\right)$. Let $H_{i}=L^{2}\left(X_{i}\right), \mathcal{D}_{i}=\left\{M_{\psi}: \psi \in L^{\infty}\left(X_{i}\right)\right\}$, $i=1, \ldots, n$, and

$$
\hat{S}_{\varphi}: \mathcal{C}_{2}\left(H_{1}, H_{2}\right) \times \cdots \times \mathcal{C}_{2}\left(H_{n-1}, H_{n}\right) \rightarrow \mathcal{C}_{2}\left(H_{1}, H_{n}\right)
$$

be the map defined by $\hat{S}_{\varphi}\left(T_{f_{1}}, \ldots, T_{f_{n}}\right)=T_{S_{\varphi}\left(f_{1}, \ldots, f_{n}\right)}$. Since $\varphi$ is a Schur multiplier, the linearisation of the map $\hat{S}_{\varphi}$ from $\left(\mathcal{C}_{2}\left(H_{1}, H_{2}\right) \odot \cdots \odot \mathcal{C}_{2}\left(H_{n-1}, H_{n}\right),\|\cdot\|_{\mathrm{h}}\right)$ into $\left(\mathcal{C}_{2}\left(H_{1}, H_{n}\right),\|\cdot\|_{\text {op }}\right)$ is bounded. (Here each of the operator spaces $\mathcal{C}_{2}\left(H_{i}, H_{i+1}\right)$ is given its opposite operator space structure arising from the inclusion $\mathcal{C}_{2}\left(H_{i}, H_{i+1}\right)$ $\subseteq \mathcal{B}\left(H_{i}, H_{i+1}\right)$.) If $a_{i} \in L^{\infty}\left(X_{i}\right), i=1, \ldots, n$, then

$$
\begin{aligned}
\hat{S}_{\varphi}\left(T_{f_{1}} M_{a_{1}}, T_{f_{2}} M_{a_{2}}, \ldots, M_{a_{n}} T_{f_{n}} M_{a_{n-1}}\right) & =\hat{S}_{\varphi}\left(T_{f_{1} a_{1}}, T_{f_{2} a_{2}}, \ldots, T_{a_{n} f_{n} a_{n-1}}\right) \\
& =T_{S_{\varphi}\left(f_{1} a_{1}, f_{2} a_{2}, \ldots, a_{n} f_{n} a_{n-1}\right)} \\
& =T_{a_{n} S_{\varphi}\left(a_{2} f_{1}, a_{3} f_{2}, \ldots, a_{n-1} f_{n-2}, f_{n}\right) a_{1}} \\
& =M_{a_{n}} \hat{S}_{\varphi}\left(M_{a_{2}} T_{f_{1}}, \ldots, T_{f_{n}}\right) M_{a_{1}} .
\end{aligned}
$$

By continuity, the map $\hat{S}_{\varphi}$ has an extension (denoted in the same way)

$$
\hat{S}_{\varphi}: \mathcal{K}\left(H_{1}, H_{2}\right) \otimes_{\mathrm{h}} \cdots \otimes_{\mathrm{h}} \mathcal{K}\left(H_{n-1}, H_{n}\right) \rightarrow \mathcal{K}\left(H_{1}, H_{n}\right)
$$

to a map with norm less than one, where the spaces $\mathcal{K}\left(H_{i}, H_{i+1}\right)$ are equipped with the operator space structure opposite to their natural operator space structure. It follows from (14) that the map

$$
\check{S}_{\varphi}: \mathcal{K}\left(H_{n-1}, H_{n}\right) \otimes_{\mathrm{h}} \cdots \otimes_{\mathrm{h}} \mathcal{K}\left(H_{1}, H_{2}\right) \rightarrow \mathcal{K}\left(H_{1}, H_{n}\right),
$$

given by

$$
\check{S}_{\varphi}\left(T_{n-1} \otimes \cdots \otimes T_{1}\right)=\hat{S}_{\varphi}\left(T_{1} \otimes \cdots \otimes T_{n-1}\right),
$$

is modular and bounded when the spaces $\mathcal{K}\left(H_{i}, H_{i+1}\right)$ are given their natural operator space structure. By Lemma 3.3. $\check{S}_{\varphi}$ is completely bounded. It follows that the second dual

$$
\check{S}_{\varphi}^{* *}: \mathcal{B}\left(H_{n-1}, H_{n}\right) \otimes_{\sigma h} \cdots \otimes_{\sigma h} \mathcal{B}\left(H_{1}, H_{2}\right) \rightarrow \mathcal{B}\left(H_{1}, H_{n}\right)
$$

is a weak* continuous map with c.b. norm less than one, which extends the map $\breve{S}_{\varphi}$. (Here $\otimes_{\sigma h}$ denotes the normal Haagerup tensor product; see e.g. [7.)

Denote by $\tilde{S}_{\varphi}$ the corresponding multilinear map

$$
\tilde{S}_{\varphi}: \mathcal{B}\left(H_{n-1}, H_{n}\right) \times \cdots \times \mathcal{B}\left(H_{1}, H_{2}\right) \rightarrow \mathcal{B}\left(H_{1}, H_{n}\right) .
$$

The map $\tilde{S}_{\varphi}$ is separately weak* continuous and hence modular.

A modification of Corollary 5.9 of 9 now implies that there exist bounded linear operators $V_{1}: H_{1} \rightarrow H_{1}^{\infty}, V_{n}: H_{n}^{\infty} \rightarrow H_{n}$ and $V_{i}: H_{i}^{\infty} \rightarrow H_{i}^{\infty}, i=2, \ldots, n-1$, such that the entries of $V_{i}$ belong to $\mathcal{D}_{i}$ and

$$
\tilde{S}_{\varphi}\left(T_{n-1}, \ldots, T_{1}\right)=V_{n}\left(T_{n-1} \otimes I\right) V_{n-1}\left(T_{n-2} \otimes I\right) \ldots\left(T_{1} \otimes I\right) V_{1} .
$$

Moreover, the operators $V_{i}$ can be chosen so that $\prod_{i=1}^{n}\left\|V_{i}\right\|<1$. Let $V_{1}=$ $\left(M_{a_{1}^{1}}, M_{a_{2}^{1}}, \ldots\right)^{\mathrm{t}}, V_{i}=\left(M_{a_{k l}^{i}}\right)$ and $V_{n}=\left(M_{a_{1}^{n}}, M_{a_{2}^{n}}, \ldots\right)$, for some $a_{1}=$ $\left(a_{1}^{1}, a_{2}^{1}, \ldots\right)^{\mathrm{t}} \in L^{\infty}\left(X_{1}, M_{1, \infty}\right), a_{n}=\left(a_{1}^{n}, a_{2}^{n}, \ldots\right) \in L^{\infty}\left(X_{n}, M_{1, \infty}\right)$ and $a_{i}=$ $\left(a_{k l}^{i}\right) \in L^{\infty}\left(X_{i}, M_{\infty}\right), i=2, \ldots, n-1$. Moreover,

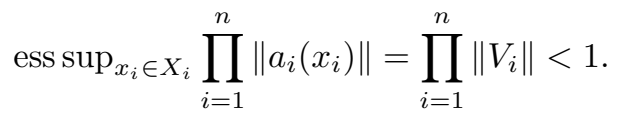


If $\xi \in L^{2}(X)$ and $\eta \in L^{2}(Y)$, denote by $\xi \otimes \eta$ the function on $X \times Y$ given by $(\xi \otimes \eta)(x, y)=\xi(x) \eta(y)$; this function gives rise by (10) to a rank one operator $T_{\xi \otimes \eta}$. Fix $\xi_{i}, \eta_{i} \in H_{i}, i=1, \ldots, n$. Then

$$
\begin{aligned}
& \tilde{S}_{\varphi}\left(T_{\xi_{n-1} \otimes \eta_{n}}, \ldots, T_{\xi_{1} \otimes \eta_{2}}\right)\left(\eta_{1}\right)=V_{n}\left(T_{\xi_{n-1} \otimes \eta_{n}} \otimes I\right) \ldots\left(T_{\xi_{1} \otimes \eta_{2}} \otimes I\right) V_{1}\left(\eta_{1}\right) \\
& =V_{n}\left(T_{\xi_{n-1} \otimes \eta_{n}} \otimes I\right) \ldots V_{2}\left(T_{\xi_{1} \otimes \eta_{2}} \otimes I\right)\left(a_{k_{1}}^{1} \eta_{1}\right)_{k_{1}} \\
& =V_{n}\left(T_{\xi_{n-1} \otimes \eta_{n}} \otimes I\right) \ldots V_{2}\left(\left(\int_{X_{1}} a_{k_{1}}^{1}\left(x_{1}\right) \xi_{1}\left(x_{1}\right) \eta_{1}\left(x_{1}\right) d x_{1}\right) \eta_{2}\right)_{k_{1}} \\
& =V_{n} \ldots\left(T_{\xi_{2} \otimes \eta_{3}} \otimes I\right)\left(\left(\sum_{k_{1}=1}^{\infty} \int_{X_{1}} a_{k_{1}}^{1}\left(x_{1}\right) \xi_{1}\left(x_{1}\right) \eta_{1}\left(x_{1}\right) d x_{1}\right) a_{k_{2}, k_{1}}^{2} \eta_{2}\right)_{k_{2}} \\
& =V_{n} \ldots V_{3}\left(\left(\sum_{k_{1}=1}^{\infty} \int_{X_{1} \times X_{2}} a_{k_{2}, k_{1}}^{2}\left(x_{2}\right) a_{k_{1}}^{1}\left(x_{1}\right)\left(\xi_{1} \eta_{1}\right)\left(x_{1}\right)\left(\xi_{2} \eta_{2}\right)\left(x_{2}\right) d x_{1} d x_{2}\right) \eta_{3}\right)_{k_{2}}
\end{aligned}
$$

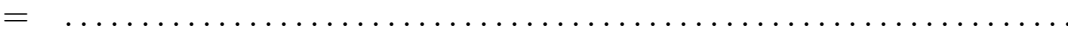

$$
\begin{aligned}
& =\sum_{k_{n}=1}^{\infty}\left(\int_{X_{1} \times \cdots \times X_{n-1}} \sum_{k_{1}, \ldots, k_{n-1}=1}^{\infty} a_{k_{n-1}, k_{n-2}}^{n-1}\left(x_{n-1}\right) \ldots a_{k_{1}}^{1}\left(x_{1}\right)\right. \\
& \left.\left.\times \xi_{1}\left(x_{1}\right) \eta_{1}\left(x_{1}\right) \ldots \xi_{n-1}\left(x_{n-1}\right)\right) d x_{1} \ldots d x_{n-1}\right) M_{a_{k_{n}}^{n}} \eta_{n} .
\end{aligned}
$$

Thus,

$$
\begin{aligned}
& \tilde{S}_{\varphi}\left(T_{\xi_{n-1} \otimes \eta_{n}}, \ldots, T_{\xi_{1} \otimes \eta_{2}}\right)\left(\eta_{1}\right)\left(x_{n}\right) \\
= & \left(\int_{X_{1} \times \cdots \times X_{n-1}} \sum_{k_{1}, \ldots, k_{n}=1}^{\infty} a_{k_{n}}^{n}\left(x_{n}\right) a_{k_{n-1}, k_{n-2}}^{n-1}\left(x_{n-1}\right) \ldots a_{k_{1}}^{1}\left(x_{1}\right)\right. \\
\times \quad & \left.\xi_{1}\left(x_{1}\right) \eta_{1}\left(x_{1}\right) \ldots \xi_{n-1}\left(x_{n-1}\right) d x_{1} \ldots d x_{n-1}\right) \eta_{n}\left(x_{n}\right) .
\end{aligned}
$$

On the other hand,

$$
\begin{aligned}
& \tilde{S}_{\varphi}\left(T_{\xi_{n-1} \otimes \eta_{n}}, \ldots, T_{\xi_{1} \otimes \eta_{2}}\right)\left(\eta_{1}\right)\left(x_{n}\right)=T_{S_{\varphi}\left(\xi_{1} \otimes \eta_{2}, \ldots, \xi_{n-1} \otimes \eta_{n}\right)}\left(\eta_{1}\right)\left(x_{n}\right) \\
= & \left(\int_{X_{1} \times \cdots \times X_{n-1}} \varphi\left(x_{1}, \ldots, x_{n-1}, x_{n}\right)\right. \\
\times \quad & \left.\xi_{1}\left(x_{1}\right) \eta_{1}\left(x_{1}\right) \ldots \xi_{n-1}\left(x_{n-1}\right) d x_{1} \ldots d x_{n-1}\right) \eta_{n}\left(x_{n}\right) .
\end{aligned}
$$

It follows that

$$
\varphi\left(x_{1}, \ldots, x_{n}\right)=a_{n}\left(x_{n}\right) a_{n-1}\left(x_{n-1}\right) \ldots a_{1}\left(x_{1}\right),
$$

for almost all $x_{1}, \ldots, x_{n}$.

(ii) $\Rightarrow$ (i) Assume that $\varphi$ is given as in (ii), where $a_{1}=\left(a_{1}^{1}, a_{2}^{1}, \ldots\right)^{\mathrm{t}} \in$ $L^{\infty}\left(X_{1}, M_{\infty, 1}\right), \quad a_{n}=\left(a_{1}^{n}, a_{2}^{n}, \ldots\right) \in L^{\infty}\left(X_{n}, M_{1, \infty}\right)$ and $a_{i}=\left(a_{k l}^{i}\right) \in$ $L^{\infty}\left(X_{i}, M_{\infty}\right), i=2, \ldots, n-1$. Let $V_{1}: H_{1} \rightarrow H_{1}^{\infty}$ be the operator corresponding to the column matrix $V_{1}=\left(M_{a_{1}^{1}}, M_{a_{2}^{1}}, \ldots\right)^{\mathrm{t}}: H_{1} \rightarrow H_{1}^{\infty}, V_{n}: H_{n}^{\infty} \rightarrow H_{n}$ be the operator corresponding to the row matrix $V_{n}=\left(M_{a_{1}^{n}}, M_{a_{2}^{n}}, \ldots\right)$ and $V_{i}: H_{i}^{\infty} \rightarrow H_{i}^{\infty}$ be the operator corresponding to the matrix $V_{i}=\left(M_{a_{k l}^{i}}\right), i=2, \ldots, n-1$. Then $\prod_{i=1}^{n}\left\|V_{i}\right\|<1$. It follows from the first part of the proof that

$$
\tilde{S}_{\varphi}\left(T_{\xi_{n-1} \otimes \eta_{n}}, \ldots, T_{\xi_{1} \otimes \eta_{2}}\right)=V_{n}\left(T_{\xi_{n-1} \otimes \eta_{n}} \otimes I\right) \ldots\left(T_{\xi_{1} \otimes \eta_{2}} \otimes I\right) V_{1},
$$

for all $\xi_{1} \in H_{1}, \eta_{n} \in H_{n}$ and $\xi_{i}, \eta_{i} \in H_{i}, i=2, \ldots, n-1$. Since the operator norm is dominated by the Hilbert-Schmidt norm, we conclude that

$$
\tilde{S}_{\varphi}\left(T_{f_{n-1}}, \ldots, T_{f_{1}}\right)=V_{n}\left(T_{f_{n-1}} \otimes I\right) \ldots\left(T_{f_{1}} \otimes I\right) V_{1},
$$


for all $f_{i} \in L^{2}\left(X_{i} \times X_{i+1}\right), i=1, \ldots, n-1$.

Let

$$
F=F_{1} \odot \cdots \odot F_{n-1} \in L^{2}\left(X_{1} \times X_{2}\right) \odot \cdots \odot L^{2}\left(X_{n-1} \times X_{n}\right),
$$

where $F_{1} \in M_{1, \infty}\left(L^{2}\left(X_{1} \times X_{2}\right)\right), F_{n-1} \in M_{\infty, 1}\left(L^{2}\left(X_{n-1} \times X_{n}\right)\right)$ and $F_{i} \in$ $M_{\infty}\left(L^{2}\left(X_{i} \times X_{i+1}\right)\right), i=2, \ldots, n-2$. Lemma 4.7 implies that

$$
T_{S_{\varphi}(F)}=V_{n}\left(T_{F_{n-1}} \otimes I\right) \ldots\left(T_{F_{1}} \otimes I\right) V_{1},
$$

where $T_{F_{i}}=\left(T_{f_{k}^{i}}\right)_{k, l}$ whenever $F_{i}=\left(f_{k l}^{i}\right)_{k, l}$. It follows that

$$
\left\|T_{S_{\varphi}(F)}\right\|_{\mathrm{op}} \leq \prod_{i=1}^{n-1}\left\|F_{i}^{t}\right\|_{\mathrm{op}} \prod_{i=1}^{n}\left\|V_{i}\right\| .
$$

Taking the infimum with respect to all representations of $F$, we conclude that $\left\|T_{S_{\varphi}(F)}\right\|_{\mathrm{op}} \leq\|F\|_{\mathrm{h}} \prod_{i=1}^{n}\left\|V_{i}\right\|$ and so $\|\varphi\|_{\mathrm{m}}<1$.

Remark. The space of all functions $\varphi\left(x_{1}, \ldots, x_{n}\right)$ satisfying condition (ii) of Theorem 3.4 can, in view of the commutativity of the $L^{\infty}\left(X_{i}\right)$ 's, be identified with the extended Haagerup tensor product $L^{\infty}\left(X_{1}\right) \otimes_{e h} L^{\infty}\left(X_{2}\right) \otimes_{e h} \ldots \otimes_{e h} L^{\infty}\left(X_{n}\right)$.

The next proposition relates our approach with a recent paper of Peller 25 on multiple operator integrals. For some fixed spectral measures, Peller defines a multiple operator integral $I_{\varphi}\left(T_{1}, \ldots, T_{n-1}\right)$ of a function $\varphi$ and an $(n-1)$-tuple of operators $\left(T_{1}, \ldots, T_{n-1}\right)$, and shows that if $\varphi$ belongs to the integral projective tensor product of the corresponding $L^{\infty}$-spaces, then $I_{\varphi}\left(T_{1}, \ldots, T_{n-1}\right)$ is well-defined and, moreover,

$$
\left\|I_{\varphi}\left(T_{1}, \ldots, T_{n-1}\right)\right\|_{\mathrm{op}} \leq\|\varphi\|_{i}\left\|T_{1}\right\|_{\mathrm{op}} \ldots\left\|T_{n-1}\right\|_{\mathrm{op}} .
$$

Recall that the integral projective tensor product $L^{\infty}\left(X_{1}\right) \hat{\otimes}_{i} \ldots \hat{\otimes}_{i} L^{\infty}\left(X_{n}\right)$ is the space of all functions $\varphi$ for which there exists a measure space $(\mathcal{T}, \nu)$ and measurable functions $g_{i}$ on $X_{i} \times \mathcal{T}$ such that

$$
\varphi\left(x_{1}, \ldots, x_{n}\right)=\int_{\mathcal{T}} g_{1}\left(x_{1}, t\right) \ldots g_{n}\left(x_{n}, t\right) d \nu(t),
$$

for almost all $x_{1} \ldots, x_{n}$, where

$$
\int_{\mathcal{T}}\left\|g_{1}(\cdot, t)\right\|_{\infty} \ldots\left\|g_{n}(\cdot, t)\right\|_{\infty} d \nu(t)<\infty .
$$

The integral projective norm $\|\varphi\|_{i}$ of $\varphi$ is the infimum of the above expressions over all representations of $\varphi$ of the form (15). It was proved by Peller in 24 that in the case where $n=2$ the integral projective tensor product $L^{\infty}\left(X_{1}\right) \hat{\otimes}_{i} L^{\infty}\left(X_{2}\right)$ coincides with the set of all Schur mulipliers. The next proposition shows that for $n>2$ the integral projective tensor product consists of multipliers. We do not know whether it coincides with the space of all Schur multipliers.

Proposition 3.5. Let $\varphi \in L^{\infty}\left(X_{1}\right) \hat{\otimes}_{i} \ldots \hat{\otimes}_{i} L^{\infty}\left(X_{n}\right)$. Then $\varphi$ is a Schur multiplier and $\|\varphi\|_{\mathrm{m}} \leq\|\varphi\|_{i}$.

Proof. Suppose that

$$
\varphi\left(x_{1}, \ldots, x_{n}\right)=\int_{\mathcal{T}} g_{1}\left(x_{1}, t\right) \ldots g_{n}\left(x_{n}, t\right) d \nu(t),
$$


for almost all $x_{1} \ldots, x_{n}$, where $(\mathcal{T}, \nu)$ is a measure space, $g_{i}$ is a measurable function on $X_{i} \times \mathcal{T}, i=1, \ldots, n$, such that

$$
\int_{\mathcal{T}}\left\|g_{1}(\cdot, t)\right\|_{\infty} \ldots\left\|g_{n}(\cdot, t)\right\|_{\infty} d \nu(t)<\infty
$$

Let $F=F_{1} \odot \cdots \odot F_{n-1}$, where $F_{1} \in M_{1, k_{1}}\left(L^{2}\left(X_{1} \times X_{2}\right)\right), F_{n-1} \in M_{k_{n-2}, 1}\left(L^{2}\left(X_{n-1}\right.\right.$ $\left.\left.\times X_{n}\right)\right)$ and $F_{i} \in M_{k_{i-1}, k_{i}}\left(L^{2}\left(X_{i} \times X_{i+1}\right)\right), i=2, \ldots, n-2$, and $\tilde{F}\left(x_{1}, x_{2}, \ldots, x_{n}\right)=$ $F\left(x_{1}, x_{2}, x_{2}, x_{3}, \ldots, x_{n}\right)$. Denoting by $M_{g_{i}(\cdot, t)}$ the multiplication operator by the function $g_{i}(\cdot, t)$, and by $M_{g_{i}(\cdot, t)} \otimes I$ the ampliation of $M_{g_{i}(\cdot, t)}$ of multiplicity $k_{i}$, we have

$$
\begin{aligned}
\left\|S_{\varphi}(F)\right\|_{\mathrm{op}} & =\left\|\int \varphi \tilde{F} d x_{2} \ldots d x_{n-1}\right\|_{\mathrm{op}} \\
& =\left\|\int\left(\int_{\mathcal{T}} g_{1}\left(x_{1}, t\right) \ldots g_{n}\left(x_{n}, t\right) d t\right) \tilde{F} d x_{2} \ldots d x_{n-1}\right\|_{\mathrm{op}} \\
& =\left\|\int_{\mathcal{T}}\left(\int g_{1}\left(x_{1}, t\right) \ldots g_{n}\left(x_{n}, t\right) d x_{2} \ldots d x_{n-1}\right) \tilde{F} d t\right\|_{\mathrm{op}} \\
& =\| \int_{\mathcal{T}}\left(\int M_{g_{1}(\cdot, t)} F_{1}\left(M_{g_{2}(\cdot, t)} \otimes I\right)\left(x_{1}, x_{2}\right) \odot \ldots\right. \\
& \left.\odot F_{n-1} M_{g_{n}(\cdot, t)}\left(x_{n-1}, x_{n}\right) d x_{2} \ldots d x_{n-1}\right) d t \|_{\mathrm{op}} \\
\leq & \int_{\mathcal{T}} \| \int M_{g_{1}(\cdot, t)} F_{1}\left(M_{g_{2}(\cdot, t)} \otimes I\right)\left(x_{1}, x_{2}\right) \odot \ldots \\
& \odot F_{n-1} M_{g_{n}(\cdot, t)}\left(x_{n-1}, x_{n}\right) d x_{2} \ldots d x_{n-1} \|_{\mathrm{op}} d t \\
& \leq \int_{\mathcal{T}}\left\|M_{g_{1}(\cdot, t)}\right\|\left\|F_{1}\right\|_{\mathrm{op}}^{o}\left\|_{g_{2}(\cdot, t)}\right\| \ldots\left\|F_{n-1}\right\|_{\mathrm{op}}^{o}\left\|M_{g_{n}(\cdot, t)}\right\| d t \\
\leq & \|\varphi\|_{i}\left\|F_{1}\right\|_{\mathrm{op}}^{o} \ldots\left\|F_{n-1}\right\|_{\mathrm{op}}^{o},
\end{aligned}
$$

where $\|\cdot\|_{o p}^{o}$ is the opposite operator norm (see Section 2). The claim follows by taking the infimum over all representations $F=F_{1} \odot \cdots \odot F_{n-1}$.

Corollary 3.6. $L^{\infty}\left(X_{1}\right) \hat{\otimes}_{i} \ldots \hat{\otimes}_{i} L^{\infty}\left(X_{n}\right) \subseteq L^{\infty}\left(X_{1}\right) \otimes_{e h} \ldots \otimes_{e h} L^{\infty}\left(X_{n}\right)$.

We finally point out another interesting open question, namely the one of characterising the class of multipliers defined by using the projective tensor norm instead of the Haagerup tensor norm in (2); equivalently, the class of multipliers obtained after replacing (2) with the weaker condition

$$
\left\|S_{\psi}\left(f_{1} \otimes \ldots \otimes f_{n}\right)\right\|_{\text {op }} \leq C\left\|f_{1}\right\|_{\text {op }} \ldots\left\|f_{n}\right\|_{\text {op }} \text { for all } f_{i} \in L^{2}\left(X_{i}\right), i=1, \ldots, n .
$$

\section{Multidimensional operator multipliers: The Definition}

In this section we generalise the notion of operator multipliers given by Kissin and Shulman 21] to the multidimensional case.

We recall the mapping $\theta_{K_{1}, K_{2}}: K_{1} \otimes K_{2} \rightarrow \mathcal{C}_{2}\left(K_{1}^{\mathrm{d}}, K_{2}\right)$, where $K_{1}$ and $K_{2}$ are Hilbert spaces, which is the unitary operator between the Hilbert spaces $K_{1} \otimes K_{2}$ and $\mathcal{C}_{2}\left(K_{1}^{\mathrm{d}}, K_{2}\right)$ given on elementary tensors by

$$
\theta_{K_{1}, K_{2}}\left(\xi_{1} \otimes \xi_{2}\right)\left(\eta_{1}^{\mathrm{d}}\right)=\left(\xi_{1}, \eta_{1}\right) \xi_{2} .
$$

Note that there is a natural identification of $\left(K_{1} \otimes K_{2}\right)^{\mathrm{d}}$ and $K_{1}^{\mathrm{d}} \otimes K_{2}^{\mathrm{d}}$. It follows that $\mathcal{C}_{2}\left(K_{1}^{\mathrm{d}}, K_{2}\right)^{\mathrm{d}}$ can be identified with $\mathcal{C}_{2}\left(K_{1}, K_{2}^{\mathrm{d}}\right)=\mathcal{C}_{2}\left(\left(K_{1}^{\mathrm{d}}\right)^{\mathrm{d}}, K_{2}^{\mathrm{d}}\right)$; we have that $\theta_{K_{1}^{\mathrm{d}}, K_{2}^{\mathrm{d}}}\left(\xi^{\mathrm{d}}\right)=\theta_{K_{1}, K_{2}}(\xi)^{\mathrm{d}}$. 
Let $H_{1}, \ldots, H_{n}$ be Hilbert spaces and $H=H_{1} \otimes \cdots \otimes H_{n}$. For any permutation $\pi$ of $\{1, \ldots, n\}$, we will identify $H$ with the tensor product $H_{\pi(1)} \otimes \cdots \otimes H_{\pi(n)}$ without explicitly mentioning this. The symbol $\xi_{j_{1}, \ldots, j_{k}}$ will denote an element of $H_{j_{1}} \otimes \cdots \otimes H_{j_{k}}$.

We define a Hilbert space $H S\left(H_{1}, \ldots, H_{n}\right)$, isometrically isomorphic to $H$. Let $H S\left(H_{1}, H_{2}\right)=\mathcal{C}_{2}\left(H_{1}^{\mathrm{d}}, H_{2}\right)$. In the case where $n$ is even, we let by induction

$$
H S\left(H_{1}, \ldots, H_{n}\right)=\mathcal{C}_{2}\left(H S\left(H_{2}, H_{3}\right)^{\mathrm{d}}, H S\left(H_{1}, H_{4}, \ldots, H_{n}\right)\right),
$$

and let

be given by

$$
\theta_{H_{1}, \ldots, H_{n}}: H \rightarrow H S\left(H_{1}, \ldots, H_{n}\right)
$$

$$
\theta_{H_{1}, \ldots, H_{n}}\left(\xi_{2,3} \otimes \xi\right)=\theta_{H S\left(H_{2}, H_{3}\right), H S\left(H_{1}, H_{4}, \ldots, H_{n}\right)}\left(\theta_{H_{2}, H_{3}}\left(\xi_{2,3}\right) \otimes \theta_{H_{1}, H_{4}, \ldots, H_{n}}(\xi)\right),
$$

where $\xi \in H_{1} \otimes H_{4} \otimes \cdots \otimes H_{n}$. In particular, we have that

$$
\theta_{H_{1}, \ldots, H_{n}}\left(\xi_{2,3} \otimes \xi\right) \theta_{H_{2}, H_{3}}\left(\eta_{2,3}\right)^{\mathrm{d}}=\left(\theta_{H_{2}, H_{3}}\left(\xi_{2,3}\right), \theta_{H_{2}, H_{3}}\left(\eta_{2,3}\right)\right) \theta_{H_{1}, H_{4}, \ldots, H_{n}}(\xi) .
$$

In the case where $n$ is odd, we let

$$
H S\left(H_{1}, \ldots, H_{n}\right)=H S\left(\mathbb{C}, H_{1}, \ldots, H_{n}\right) .
$$

If $K$ is a Hilbert space, we will identify $\mathcal{C}_{2}\left(\mathbb{C}^{\mathrm{d}}, K\right)$ with $K$ via the map $S \rightarrow S\left(1^{\mathrm{d}}\right)$. Thus, $H S\left(H_{1}, \ldots, H_{n}\right)$ can, in the case of odd $n$, be defined inductively by letting $H S\left(H_{1}\right)=H_{1}$ and

$$
H S\left(H_{1}, \ldots, H_{n}\right)=\mathcal{C}_{2}\left(H S\left(H_{1}, H_{2}\right)^{\mathrm{d}}, H S\left(H_{3}, \ldots, H_{n}\right)\right) .
$$

The isomorphism $\theta_{H_{1}, \ldots, H_{n}}$ is in this case given by

$$
\theta_{H_{1}, \ldots, H_{n}}(\xi)=\theta_{\mathbb{C}, H_{1}, \ldots, H_{n}}(1 \otimes \xi) .
$$

We will usually omit the subscripts and write simply $\theta$, when the corresponding Hilbert spaces are understood.

Lemma 4.1. (i) Assume $n$ is even. Let $\xi \in H$ be of the form $\xi=\xi_{1,2} \otimes \cdots \otimes \xi_{n-1, n}$. If $\eta_{i, i+1} \in H_{i} \otimes H_{i+1}$ ( $i$ even), then

$$
\theta(\xi)\left(\theta\left(\eta_{2,3}^{\mathrm{d}}\right)\right) \ldots\left(\theta\left(\eta_{n-2, n-1}^{\mathrm{d}}\right)\right)=\theta\left(\xi_{n-1, n}\right) \theta\left(\eta_{n-2, n-1}^{\mathrm{d}}\right) \ldots \theta\left(\xi_{3,4}\right) \theta\left(\eta_{2,3}^{\mathrm{d}}\right) \theta\left(\xi_{1,2}\right) .
$$

(ii) Assume $n$ is odd. Let $\xi \in H$ be of the form $\xi=\xi_{1} \otimes \xi_{2,3} \otimes \cdots \otimes \xi_{n-1, n}$. If $\eta_{i, i+1} \in H_{i} \otimes H_{i+1}$ ( $i$ odd), then

$$
\theta(\xi)\left(\theta\left(\eta_{1,2}^{\mathrm{d}}\right)\right)\left(\theta\left(\eta_{3,4}^{\mathrm{d}}\right)\right) \ldots\left(\theta\left(\eta_{n-2, n-1}^{\mathrm{d}}\right)\right)=\theta\left(\xi_{n-1, n}\right) \theta\left(\eta_{n-2, n-1}^{\mathrm{d}}\right) \ldots \theta\left(\eta_{1,2}^{\mathrm{d}}\right)\left(\xi_{1}\right) .
$$

Proof. (i) Assume first that $\xi_{i-1, i}=\xi_{i-1} \otimes \xi_{i}$ and $\eta_{i, i+1}=\eta_{i} \otimes \eta_{i+1}$ ( $i$ even). Fix $\eta_{1}^{\mathrm{d}} \in H_{1}^{\mathrm{d}}$. The image of $\eta_{1}^{\mathrm{d}}$ under the operator on the right-hand side of the identity in (i) is

$$
\left(\xi_{1}, \eta_{1}\right)\left(\xi_{2}, \eta_{2}\right) \ldots\left(\xi_{n-1}, \eta_{n-1}\right) \xi_{n}
$$

On the other hand, the image of $\eta_{1}^{\mathrm{d}}$ under the operator on the left-hand side is

$$
\begin{aligned}
& \left(\theta_{H_{2}, H_{3}}\left(\xi_{2} \otimes \xi_{3}\right), \theta_{H_{2}, H_{3}}\left(\eta_{2} \otimes \eta_{3}\right)\right) \\
\times & \theta_{H_{1}, H_{4}, \ldots, H_{n}}\left(\xi_{1} \otimes \xi_{4} \otimes \cdots \otimes \xi_{n}\right)\left(\theta\left(\eta_{4,5}\right)^{\mathrm{d}}\right) \ldots\left(\theta\left(\eta_{n-2, n-1}\right)^{\mathrm{d}}\right)\left(\eta_{1}^{\mathrm{d}}\right) \\
= & \left(\xi_{2}, \eta_{2}\right)\left(\xi_{3}, \eta_{3}\right) \\
\times & \theta_{H_{1}, H_{4}, \ldots, H_{n}}\left(\xi_{1} \otimes \xi_{4} \otimes \cdots \otimes \xi_{n}\right)\left(\theta\left(\eta_{4,5}\right)^{\mathrm{d}}\right) \ldots\left(\theta\left(\eta_{n-2, n-1}\right)^{\mathrm{d}}\right)\left(\eta_{1}^{\mathrm{d}}\right) .
\end{aligned}
$$

By induction, (i) holds in the case of elementary tensors. 
By linearity, (i) holds for finite sums of elementary tensors. Using continuity arguments and the fact that the operator norm is dominated by the Hilbert-Schmidt norm, one can easily prove that $(i)$ holds for general $\xi$ and $\eta_{i, i+1}$.

We define a representation $\sigma_{H}$ of $B(H)$ on $H S\left(H_{1}, \ldots, H_{n}\right)$ by letting

$$
\sigma_{H}(A) \theta(\xi)=\theta(A \xi)
$$

clearly, $\sigma_{H}$ is unitarily equivalent to the identity representation of $B(H)$. If $H_{1}, \ldots$, $H_{n}$ are clear from the context we will simply write $\sigma$ in the place of $\sigma_{H}$. If $\mathcal{A}_{1}, \ldots, \mathcal{A}_{n}$ are $C^{*}$-algebras, $\pi_{1}, \ldots, \pi_{n}$ corresponding representations on $H_{1}, \ldots, H_{n}$, and $\pi=\pi_{1} \otimes \cdots \otimes \pi_{n}$ we let

$$
\sigma_{\pi}=\sigma_{H} \circ \pi
$$

thus, $\sigma_{\pi}$ is a representation of $\mathcal{A}_{1} \otimes \cdots \otimes \mathcal{A}_{n}$ on $H S\left(H_{1}, \ldots, H_{n}\right)$, unitarily equivalent to $\pi$.

Lemma 4.2. Let $A_{i} \in B\left(H_{i}\right), i=1, \ldots, n$, and $A=A_{1} \otimes \cdots \otimes A_{n}$.

(i) Assume $n$ is even. Let $\xi_{i-1, i} \in H_{i-1} \otimes H_{i}, \eta_{i, i+1} \in H_{i} \otimes H_{i+1}$ (i even). If $\xi=\xi_{1,2} \otimes \cdots \otimes \xi_{n-1, n}$, then

$$
\begin{aligned}
& \sigma(A)(\theta(\xi))\left(\theta\left(\eta_{2,3}^{\mathrm{d}}\right)\right) \ldots\left(\theta\left(\eta_{n-2, n-1}^{\mathrm{d}}\right)\right) \\
= & A_{n} \theta\left(\xi_{n-1, n}\right) A_{n-1}^{\mathrm{d}} \theta\left(\eta_{n-2, n-1}\right)^{\mathrm{d}} A_{n-2} \ldots A_{2} \theta\left(\xi_{1,2}\right) A_{1}^{\mathrm{d}} \\
= & A_{n} \theta(\xi)\left(\theta\left(\left(A_{2}^{*} \otimes A_{3}^{*}\left(\eta_{2,3}\right)\right)^{\mathrm{d}}\right)\right) \ldots\left(\theta\left(\left(A_{n-2}^{*} \otimes A_{n-1}^{*}\left(\eta_{n-2, n-1}\right)\right)^{\mathrm{d}}\right)\right) A_{1}^{\mathrm{d}} .
\end{aligned}
$$

(ii) Assume $n$ is odd. Let $\xi_{1} \in H_{1}, \xi_{i-1, i} \in H_{i-1} \otimes H_{i}, \eta_{i, i+1} \in H_{i} \otimes H_{i+1}$ (i odd). If $\xi=\xi_{1} \otimes \xi_{2,3} \otimes \cdots \otimes \xi_{n-1, n}$, then

$$
\begin{aligned}
& \sigma(A)(\theta(\xi))\left(\theta\left(\eta_{1,2}^{\mathrm{d}}\right)\right) \ldots\left(\theta\left(\eta_{n-2, n-1}^{\mathrm{d}}\right)\right) \\
= & A_{n} \theta\left(\xi_{n-1, n}\right) A_{n-1}^{\mathrm{d}} \theta\left(\eta_{n-2, n-1}\right)^{\mathrm{d}} A_{n-2} \ldots A_{2}^{\mathrm{d}} \theta\left(\eta_{1,2}^{\mathrm{d}}\right)\left(A_{1} \xi_{1}\right) \\
= & A_{n} \theta(\xi)\left(\theta\left(\left(A_{1}^{*} \otimes A_{2}^{*}\left(\eta_{1,2}\right)\right)^{\mathrm{d}}\right)\right) \ldots\left(\theta\left(\left(A_{n-2}^{*} \otimes A_{n-1}^{*}\left(\eta_{n-2, n-1}\right)\right)^{\mathrm{d}}\right)\right) .
\end{aligned}
$$

Proof. (i) Let first $n=2$. If $\eta^{\mathrm{d}} \in H_{1}^{\mathrm{d}}$ and $\xi=\xi_{1} \otimes \xi_{2}$, then

$$
\begin{aligned}
\sigma(A)(\theta(\xi))\left(\eta^{\mathrm{d}}\right) & =\theta\left(A_{1} \xi_{1} \otimes A_{2} \xi_{2}\right)\left(\eta^{\mathrm{d}}\right)=\left(A_{1} \xi_{1}, \eta\right) A_{2} \xi_{2} \\
& =\left(\xi_{1}, A_{1}^{*} \eta\right) A_{2} \xi_{2}=A_{2} \theta\left(\xi_{1} \otimes \xi_{2}\right)\left(\left(A_{1}^{*} \eta\right)^{\mathrm{d}}\right) \\
& =A_{2} \theta\left(\xi_{1} \otimes \xi_{2}\right) A_{1}^{\mathrm{d}}\left(\eta^{\mathrm{d}}\right)=A_{2} \theta(\xi) A_{1}^{\mathrm{d}}\left(\eta^{\mathrm{d}}\right) .
\end{aligned}
$$

It follows by linearity and continuity that $\sigma(A)(\theta(\xi))=A_{2} \theta(\xi) A_{1}^{\mathrm{d}}$, for every $\xi \in$ $H_{1} \otimes H_{2}$. Using Lemma 4.1 (i) we now obtain

$$
\begin{aligned}
& \sigma(A)(\theta(\xi))\left(\theta\left(\eta_{2,3}\right)^{\mathrm{d}}\right) \ldots\left(\theta\left(\eta_{n-2, n-1}^{\mathrm{d}}\right)\right) \\
= & \theta\left(\left(A_{1} \otimes \cdots \otimes A_{n}\right)(\xi)\right)\left(\theta\left(\eta_{2,3}\right)^{\mathrm{d}}\right) \ldots\left(\theta\left(\eta_{n-2, n-1}^{\mathrm{d}}\right)\right) \\
= & \theta\left(\left(A_{n-1} \otimes A_{n}\right)\left(\xi_{n-1, n}\right)\right) \theta\left(\eta_{n-2, n-1}^{\mathrm{d}}\right) \ldots \\
& \ldots \theta\left(\left(A_{3} \otimes A_{4}\right)\left(\xi_{3,4}\right)\right) \theta\left(\eta_{2,3}^{\mathrm{d}}\right) \theta\left(\left(A_{1} \otimes A_{2}\right)\left(\xi_{1,2}\right)\right) \\
= & A_{n} \theta\left(\xi_{n-1, n}\right) A_{n-1}^{\mathrm{d}} \theta\left(\eta_{n-2, n-1}\right)^{\mathrm{d}} A_{n-2} \ldots A_{3}^{\mathrm{d}} \theta\left(\eta_{2,3}\right)^{\mathrm{d}} A_{2} \theta\left(\xi_{1,2}\right) A_{1}^{\mathrm{d}} \\
= & \left.\left.A_{n} \theta(\xi)\left(\theta\left(\left(A_{2}^{*} \otimes A_{3}^{*}\right)\left(\eta_{2,3}\right)\right)^{\mathrm{d}}\right)\right) \ldots\left(\theta\left(\left(A_{n-2}^{*} \otimes A_{n-1}^{*}\right)\left(\eta_{n-2, n-1}\right)\right)^{\mathrm{d}}\right)\right) A_{1}^{\mathrm{d}} .
\end{aligned}
$$


(ii) By Lemma 4.1 (ii),

$$
\begin{aligned}
& \sigma(A)(\theta(\xi))\left(\theta\left(\eta_{1,2}\right)^{\mathrm{d}}\right) \ldots\left(\theta\left(\eta_{n-2, n-1}\right)^{\mathrm{d}}\right) \\
= & \theta\left(\left(A_{1} \otimes \ldots A_{n}\right)(\xi)\right)\left(\theta\left(\eta_{1,2}\right)^{\mathrm{d}}\right) \ldots\left(\theta\left(\eta_{n-2, n-1}\right)^{\mathrm{d}}\right) \\
= & \theta\left(\left(A_{n-1} \otimes A_{n}\right)\left(\xi_{n-1, n}\right)\right) \theta\left(\eta_{n-2, n-1}^{\mathrm{d}}\right) \ldots \theta\left(\eta_{1,2}^{\mathrm{d}}\right)\left(A_{1} \xi_{1}\right) \\
= & A_{n} \theta\left(\xi_{n-1, n}\right) A_{n-1}^{\mathrm{d}} \theta\left(\eta_{n-2, n-1}\right)^{\mathrm{d}} A_{n-2} \ldots A_{2}^{\mathrm{d}} \theta\left(\eta_{1,2}^{\mathrm{d}}\right)\left(A_{1} \xi_{1}\right) \\
= & \left.\left.A_{n} \theta(\xi)\left(\theta\left(\left(A_{1}^{*} \otimes A_{2}^{*}\right)\left(\eta_{1,2}\right)\right)^{\mathrm{d}}\right)\right) \ldots\left(\theta\left(\left(A_{n-2}^{*} \otimes A_{n-1}^{*}\right)\left(\eta_{n-2, n-1}\right)\right)^{\mathrm{d}}\right)\right) .
\end{aligned}
$$

Let $H_{1}, \ldots, H_{n}$ be Hilbert spaces. If $n$ is even, we let

$$
\Gamma\left(H_{1}, \ldots, H_{n}\right)=\left(H_{1} \otimes H_{2}\right) \odot\left(H_{2}^{\mathrm{d}} \otimes H_{3}^{\mathrm{d}}\right) \odot\left(H_{3} \otimes H_{4}\right) \odot \cdots \odot\left(H_{n-1} \otimes H_{n}\right) .
$$

If $n$ is odd, we let

$$
\Gamma\left(H_{1}, \ldots, H_{n}\right)=\left(H_{1}^{\mathrm{d}} \otimes H_{2}^{\mathrm{d}}\right) \odot\left(H_{2} \otimes H_{3}\right) \odot\left(H_{3}^{\mathrm{d}} \otimes H_{4}^{\mathrm{d}}\right) \odot \cdots \odot\left(H_{n-1} \otimes H_{n}\right) .
$$

After identifying $\mathbb{C} \otimes H_{1}$ with $H_{1}$, for $n$ odd we have the identification

$$
\Gamma\left(\mathbb{C}, H_{1}, \ldots, H_{n}\right) \equiv H_{1} \odot \Gamma\left(H_{1}, \ldots, H_{n}\right) .
$$

Fix $\varphi \in B(H)$. We define a mapping $S_{\varphi}$ on $\Gamma\left(H_{1}, \ldots, H_{n}\right)$ taking values in $\mathcal{B}\left(H_{1}^{\mathrm{d}}, H_{n}\right)$ in the case $n$ is even, and in $\mathcal{B}\left(H_{1}, H_{n}\right)$, in the case $n$ is odd. First let $n$ be even. On elementary tensors

$$
\zeta=\xi_{1,2} \otimes \eta_{2,3}^{\mathrm{d}} \otimes \xi_{3,4} \otimes \cdots \otimes \xi_{n-1, n} \in \Gamma\left(H_{1}, \ldots, H_{n}\right),
$$

we let

$$
S_{\varphi}(\zeta)=\sigma(\varphi) \theta\left(\xi_{1,2} \otimes \xi_{3,4} \otimes \cdots \otimes \xi_{n-1, n}\right)\left(\theta\left(\eta_{2,3}^{\mathrm{d}}\right)\right) \ldots\left(\theta\left(\eta_{n-2, n-1}^{\mathrm{d}}\right)\right)
$$

and extend $S_{\varphi}$ on the whole of $\Gamma\left(H_{1}, \ldots, H_{n}\right)$ by linearity. Note that the values of $S_{\varphi}$ are Hilbert-Schmidt operators. Now assume $n$ is odd. Let $\zeta \in \Gamma\left(H_{1}, \ldots, H_{n}\right)$ and $\xi_{1} \in H_{1}$. Then

$$
\xi_{1} \otimes \zeta \in H_{1} \odot \Gamma\left(H_{1}, \ldots, H_{n}\right)=\Gamma\left(\mathbb{C}, H_{1}, \ldots, H_{n}\right) .
$$

We let $S_{\varphi}(\zeta)$ be the operator defined on $H_{1}$ by

$$
S_{\varphi}(\zeta)\left(\xi_{1}\right)=S_{1 \otimes \varphi}\left(\xi_{1} \otimes \zeta\right) .
$$

Note that $S_{1 \otimes \varphi}\left(\xi_{1} \otimes \zeta\right)$ is an element of $\mathcal{C}_{2}\left(\mathbb{C}^{d}, H_{n}\right)$, which can be identified with $H_{n}$ in a natural way. In this way, $S_{\varphi}(\zeta)\left(\xi_{1}\right)$ can be viewed as an element of $H_{n}$. It is clear that the operator $S_{\varphi}(\zeta): H_{1} \rightarrow H_{n}$ is linear. We moreover claim that $S_{\varphi}(\zeta)$ is bounded. Let

$$
\zeta=\eta_{1,2}^{\mathrm{d}} \otimes \cdots \otimes \xi_{n-1, n} \in \Gamma\left(H_{1}, \ldots, H_{n}\right),
$$

and $\xi_{1} \in H_{1}$. Then $S_{\varphi}(\zeta)$ is a bounded operator and

$$
\left\|S_{\varphi}(\zeta)\right\|_{\mathcal{B}\left(H_{1}, H_{n}\right)} \leq\|\varphi\|_{\mathcal{B}(H)}\left\|\eta_{1,2}\right\| \ldots\left\|\eta_{n-2, n-1}\right\|\left\|\xi_{2,3}\right\| \ldots\left\|\xi_{n-1, n}\right\| .
$$


In fact, assuming for simplicity that $n=5$ we have

$$
\begin{aligned}
& \left\|S_{\varphi}(\zeta)\left(\xi_{1}\right)\right\|=\left\|S_{1 \otimes \varphi}\left(\xi_{1} \otimes \zeta\right)\right\| \\
= & \left\|\sigma(1 \otimes \varphi) \theta\left(\left(1 \otimes \xi_{1}\right) \otimes \xi_{2,3} \otimes \xi_{4,5}\right)\left(\theta\left(\eta_{1,2}^{\mathrm{d}}\right)\right)\left(\theta\left(\eta_{3,4}^{\mathrm{d}}\right)\right)\right\| \\
\leq & \left\|\sigma(1 \otimes \varphi) \theta\left(\left(1 \otimes \xi_{1}\right) \otimes \xi_{2,3} \otimes \xi_{4,5}\right)\left(\theta\left(\eta_{1,2}^{\mathrm{d}}\right)\right)\right\|_{\mathrm{op}}\left\|\left(\theta\left(\eta_{3,4}^{\mathrm{d}}\right)\right)\right\| \\
\leq & \left\|\sigma(1 \otimes \varphi) \theta\left(\left(1 \otimes \xi_{1}\right) \otimes \xi_{2,3} \otimes \xi_{4,5}\right)\right\|\left\|_{\mathrm{op}}\right\| \eta_{1,2}\|\| \eta_{3,4} \| \\
\leq & \|\varphi\|_{\mathcal{B}(H)}\left\|\xi_{1}\right\|\left\|\xi_{2,3}\right\|\left\|\xi_{4,5}\right\|\left\|\eta_{1,2}\right\|\left\|\eta_{3,4}\right\| \\
= & \|\varphi\|_{\mathcal{B}(H)}\|\zeta\|_{2, \wedge}\left\|\xi_{1}\right\| .
\end{aligned}
$$

Before proceeding, we identify two norms with which the space $\Gamma\left(H_{1}, \ldots, H_{n}\right)$ can be equipped. The first norm on $\Gamma\left(H_{1}, \ldots, H_{n}\right)$ is the projective tensor norm $\|\cdot\|_{2, \wedge}$, where each of the terms $H_{i} \otimes H_{i+1}$ (resp. $\left.H_{i-1}^{\mathrm{d}} \otimes H_{i}^{\mathrm{d}}\right)$ is given its Hilbert space norm. In order to describe the second norm, note that if $K_{1}$ and $K_{2}$ are Hilbert spaces, then $K_{1} \otimes K_{2}$ can be endowed with an operator space structure by letting

$$
\left\|\left(\xi_{i j}\right)\right\|=\left\|\theta\left(\xi_{j i}\right)\right\|_{M_{m}\left(\mathcal{B}\left(K_{1}^{\mathrm{d}}, K_{2}\right)\right)}, \quad\left(\xi_{i j}\right) \in M_{m}\left(K_{1} \otimes K_{2}\right) .
$$

We write $\left(K_{1} \otimes K_{2}\right)_{\mathrm{op}}^{o}$ for this operator space. Note that this is the opposite operator space structure on $\mathcal{C}_{2}\left(K_{1}^{\mathrm{d}}, K_{2}\right) \subseteq \mathcal{B}\left(k_{1}^{d}, k_{2}\right)$, after the identification of $K_{1} \otimes K_{2}$ and $\mathcal{C}_{2}\left(K_{1}^{\mathrm{d}}, K_{2}\right)$. The norm $\|\cdot\|_{\mathrm{h}}$ is the Haagerup norm on $\Gamma\left(H_{1}, \ldots, H_{n}\right)$ when $\Gamma\left(H_{1}, \ldots, H_{n}\right)$ is viewed as the algebraic tensor product of the operator spaces $\left(H_{i} \otimes H_{i+1}\right)_{\mathrm{op}}^{o}\left(\operatorname{resp} .\left(H_{i-1}^{\mathrm{d}} \otimes H_{i}^{\mathrm{d}}\right)_{\mathrm{op}}^{o}\right)$. Thus, the norm $\|u\|_{\mathrm{h}}$ of a finite sum $u=$ $\sum_{i} \xi_{1,2}^{i} \otimes \ldots \otimes \xi_{n-1, n}^{i} \in \Gamma\left(H_{1}, \ldots, H_{n}\right)$ of elementary tensors equals the Haagerup norm of the element $\sum_{i} \theta\left(\xi_{n-1, n}^{i}\right) \otimes \ldots \otimes \theta\left(\xi_{1,2}^{i}\right)$.

Remark 4.3. For each $\varphi \in B(H)$ and each $\zeta \in \Gamma\left(H_{1}, \ldots, H_{n}\right)$, we have

$$
\left\|S_{\varphi}(\zeta)\right\|_{\text {op }} \leq\|\varphi\|_{\mathcal{B}(H)}\|\zeta\|_{2, \wedge} .
$$

Proof. In the case where $n$ is odd and $\zeta$ is an elementary tensor, the inequality coincides with (16). In the case that $n$ is even and $\zeta$ is an elementary tensor, this is verified similarly. The general case now follows by linearity.

Definition 4.4. An element $\varphi \in B\left(H_{1} \otimes \cdots \otimes H_{n}\right)$ is called a concrete (operator) multiplier if there exists $C>0$ such that

$$
\left\|S_{\varphi}(\zeta)\right\|_{\text {op }} \leq C\|\zeta\|_{\mathrm{h}}, \text { for each } \zeta \in \Gamma\left(H_{1}, \ldots, H_{n}\right) .
$$

The smallest such $C$ is denoted by $\|\varphi\|_{\mathrm{m}}$.

Let $\mathcal{A}_{1}, \ldots, \mathcal{A}_{n}$ be $C^{*}$-algebras and $\pi_{1}, \ldots, \pi_{n}$ be corresponding representations on the Hilbert spaces $H_{1}, \ldots, H_{n}$. An element $\varphi \in \mathcal{A}_{1} \otimes \cdots \otimes \mathcal{A}_{n}$ is called a $\left(\pi_{1}, \ldots, \pi_{n}\right)$-multiplier if $\left(\pi_{1} \otimes \cdots \otimes \pi_{n}\right)(\varphi)$ is a concrete multiplier. We denote the set of all $\left(\pi_{1}, \ldots, \pi_{n}\right)$-multipliers in $\mathcal{A}_{1} \otimes \cdots \otimes \mathcal{A}_{n}$ by $\mathbf{M}_{\pi_{1}, \ldots, \pi_{n}}\left(\mathcal{A}_{1}, \ldots \mathcal{A}_{n}\right)$. If $\varphi \in \mathbf{M}_{\pi_{1}, \ldots, \pi_{n}}\left(\mathcal{A}_{1}, \ldots \mathcal{A}_{n}\right)$, we let $\|\varphi\|_{\pi_{1}, \ldots, \pi_{n}}=\left\|\left(\pi_{1} \otimes \cdots \otimes \pi_{n}\right)(\varphi)\right\|_{\mathrm{m}}$.

The element $\varphi \in \mathcal{A}_{1} \otimes \cdots \otimes \mathcal{A}_{n}$ is called a universal multiplier if $\varphi$ is a $\left(\pi_{1}, \ldots, \pi_{n}\right)$ multiplier for all representations $\pi_{i}$ of $\mathcal{A}_{i}, i=1, \ldots, n$. We denote by $\mathbf{M}\left(\mathcal{A}_{1}, \ldots \mathcal{A}_{n}\right)$ the set of all universal multipliers in $\mathcal{A}_{1} \otimes \cdots \otimes \mathcal{A}_{n}$.

Remark 4.5. In the case $n=2$, Definition 4.4 reduces to the definition of $\mathcal{C}_{\infty^{-}}$ multipliers studied in [21]. 
Next we show that an element $\varphi \in L^{\infty}\left(X_{1}\right) \otimes \ldots \otimes L^{\infty}\left(X_{n}\right) \subset L^{\infty}\left(X_{1} \times \ldots \times X_{n}\right)$ is a Schur multiplier as defined in Section 3 if and only if $\varphi$ is a $\left(\pi_{1}, \ldots, \pi_{n}\right)$ multiplier, where $\pi_{i}$ is the canonical representation of $L^{\infty}\left(X_{i}\right)$ on $L^{2}\left(X_{i}\right)$ acting by multiplication.

Let $\mathcal{A}$ be a commutative $C^{*}$-algebra with maximal ideal space $X$, acting on a Hilbert space $H$. It is well known that, up to unitary equivalence, $H=\bigoplus_{\gamma \in \Gamma} H_{\gamma}$, where $H_{\gamma}=L_{2}\left(X, \mu_{\gamma}\right)$ is invariant under $\mathcal{A}$ for each $\gamma \in \Gamma$, and an element $f \in \mathcal{A}$ acts as on $H_{\gamma}$ by multiplication. Let $j: H \rightarrow H$ be given by $\left\{\xi_{\gamma}(\lambda)\right\} \mapsto\left\{\overline{\xi_{\gamma}(\lambda)}\right\}$. Then $V=\partial j$ is a unitary operator from $H$ to $H^{\mathrm{d}}$ such that $A^{\mathrm{d}}=V A V^{-1}$ for all $A \in \mathcal{A}$. If $K$ is another Hilbert space, then $U(T)=T V\left(\operatorname{resp} . W(S)=V^{-1} S\right)$ is an isometry from $\mathcal{C}_{2}\left(H^{\mathrm{d}}, K\right)$ to $\mathcal{C}_{2}(H, K)$ (resp. from $\mathcal{C}_{2}\left(K, H^{\mathrm{d}}\right)$ to $\left.\mathcal{C}_{2}(K, H)\right)$.

Let $\mathcal{A}_{1}, \ldots, \mathcal{A}_{n}$ be commutative $C^{*}$-algebras and let $\pi_{1}, \ldots, \pi_{n}$ be corresponding representations on $H_{1}, \ldots, H_{n}$ and $\pi=\pi_{1} \otimes \ldots \otimes \pi_{n}$. Let $V_{i}: H_{i} \rightarrow H_{i}^{\mathrm{d}}$ be the unitary operator defined above with the property $\pi_{i}\left(a_{i}\right)^{\mathrm{d}}=V_{i} \pi_{i}\left(a_{i}\right) V_{i}^{-1}$ for each $a_{i} \in \mathcal{A}_{i}, i=1, \ldots, n$. Define $U_{i, k}: \mathcal{C}_{2}\left(H_{i}^{\mathrm{d}}, H_{k}\right) \rightarrow \mathcal{C}_{2}\left(H_{i}, H_{k}\right)$ and $W_{i, k}$ : $\mathcal{C}_{2}\left(H_{i}, H_{k}^{\mathrm{d}}\right) \rightarrow \mathcal{C}_{2}\left(H_{i}, H_{k}\right)$ to be $U_{i, k}(T)=T V_{i}$ and $W_{i, k}(S)=V_{k}^{-1} S$. Then for $\varphi \in \mathcal{A}_{1} \otimes \cdots \otimes \mathcal{A}_{n}$, the mapping $S_{\pi(\varphi)}$ can be identified with a mapping $\check{S}_{\pi(\varphi)}$ from $\mathcal{C}_{2}\left(H_{1}, H_{2}\right) \odot \mathcal{C}_{2}\left(H_{2}, H_{3}\right) \odot \ldots \odot \mathcal{C}_{2}\left(H_{n-1}, H_{n}\right)$ into $\mathcal{B}\left(H_{1}, H_{n}\right)$ such that whenever $\varphi=a_{1} \otimes \ldots \otimes a_{n}$ is an elementary tensor, then

$$
\check{S}_{\pi(\varphi)}\left(R_{1} \otimes \ldots \otimes R_{n-1}\right)=\pi_{n}\left(a_{n}\right) R_{n-1} \pi_{n-1}\left(a_{n-1}\right) R_{n-2} \ldots R_{1} \pi_{1}\left(a_{1}\right) .
$$

In fact, let $\mathcal{U}=U_{1,2} \theta_{H_{1}, H_{2}} \otimes W_{2,3} \theta_{H_{2}, H_{3}} \otimes \ldots \otimes U_{n-1, n} \theta_{H_{n-1}, H_{n}}$ if $n$ is even and $\mathcal{U}=W_{1,2} \theta_{H_{1}, H_{2}} \otimes U_{2,3} \theta_{H_{2}, H_{3}} \otimes \ldots \otimes U_{n-1, n} \theta_{H_{n-1}, H_{n}}$ if $n$ is odd. Then $\mathcal{U}$ maps the space $\Gamma\left(H_{1}, H_{2}, \ldots, H_{n}\right)$ onto $\mathcal{C}_{2}\left(H_{1}, H_{2}\right) \odot \mathcal{C}_{2}\left(H_{2}, H_{3}\right) \odot \ldots \odot \mathcal{C}_{2}\left(H_{n-1}, H_{n}\right)$ and is an isometry with respect to the norm $\|\cdot\|_{\mathrm{h}}$ (this norm being defined on the algebraic tensor product of the $\mathcal{C}_{2}$-spaces again as the Haagerup norm, where each of the $\mathcal{C}_{2}$-spaces is equipped with its opposite operator space structure). Let

$$
\check{S}_{\pi(\varphi)}=U_{1, n} S_{\pi(\varphi)} \mathcal{U}^{-1}
$$

in the case that $n$ is even and

$$
\check{S}_{\pi(\varphi)}=S_{\pi(\varphi)} \mathcal{U}^{-1}
$$

in the case that $n$ is odd. Assume that $\varphi=a_{1} \otimes \ldots \otimes a_{n}$. Then, in the case where $n$ is even, we have

$$
\begin{aligned}
& \check{S}_{\pi(\varphi)}\left(R_{1} \otimes \ldots \otimes R_{n-1}\right) \\
& =U_{1, n} S_{\pi(\varphi)} \mathcal{U}^{-1}\left(R_{1} \otimes \ldots \otimes R_{n-1}\right) \\
& =U_{1, n}\left(\pi_{n}\left(a_{n}\right) U_{n-1, n}^{-1}\left(R_{n-1}\right) \pi_{n-1}\left(a_{n-1}\right)^{\mathrm{d}} W_{n-2, n-1}\left(R_{n-2}\right) \ldots \pi_{1}\left(a_{1}\right)^{\mathrm{d}}\right) \\
& =\pi_{n}\left(a_{n}\right) R_{n-1} V_{n-1}^{-1} \pi_{n-1}\left(a_{n-1}\right)^{\mathrm{d}} V_{n-1} R_{n-2} \ldots R_{1} V_{1}^{-1} \pi_{1}\left(a_{1}\right)^{\mathrm{d}} V_{1} \\
& =\pi_{n}\left(a_{n}\right) R_{n-1} \pi_{n-1}\left(a_{n-1}\right) R_{n-2} \ldots R_{1} \pi_{1}\left(a_{1}\right) .
\end{aligned}
$$

In the case where $n$ is odd one shows in a similar way that (17) holds.

Now let $\left(X_{i}, \mu_{i}\right)$ be a standard measure space, $\mathcal{A}_{i}=L^{\infty}\left(X_{i}\right)$ and let $\pi_{i}$ be the representation of $\mathcal{A}_{i}$ on $L^{2}\left(X_{i}\right)$ given by $\left(\pi_{i}(f) \xi\right)(x)=f(x) \xi(x), \xi \in L^{2}\left(X_{i}\right)$, $i=1, \ldots, n$.

Suppose $n$ is even. In this case $\check{S}_{\pi(\varphi)}\left(R_{1} \otimes \cdots \otimes R_{n-1}\right)$ is an element of $\mathcal{C}_{2}\left(H_{1}, H_{n}\right)$. Using (18) and the identification $\psi_{k, l}: f \mapsto T_{f}$ of $L_{2}\left(X_{k}, X_{l}\right)$ with the class of 
Hilbert-Schmidt operators from $L_{2}\left(X_{k}\right)$ to $L_{2}\left(X_{l}\right)$, where

$$
\left(T_{f} \xi\right)(y)=\int_{X_{k}} f(x, y) \xi(x) d x, \quad f \in L_{2}\left(X_{k} \times X_{l}\right), \xi \in L^{2}\left(X_{k}\right), y \in X_{l},
$$

we obtain that if $f_{1} \otimes \ldots \otimes f_{n-1} \in \Gamma\left(X_{1}, \ldots, X_{n}\right)$ and $\varphi$ is an elementary tensor, then

$$
\begin{aligned}
& \psi_{1, n}^{-1}\left(\check{S}_{\pi(\varphi)}\left(\psi_{1,2} \otimes \ldots \otimes \psi_{n-1, n}\right)\left(f_{1} \otimes \ldots \otimes f_{n-1}\right)\right)\left(x_{1}, x_{n}\right) \\
& =\int_{X_{2} \times \ldots \times X_{n-1}} \varphi\left(x_{1}, \ldots, x_{n}\right) f_{1}\left(x_{1}, x_{2}\right) \ldots f_{n-1}\left(x_{n-1}, x_{n}\right) d x_{2} \ldots d x_{n-1} \\
& =S_{\varphi}\left(f_{1} \otimes \ldots \otimes f_{n-1}\right)\left(x_{1}, x_{n}\right) .
\end{aligned}
$$

By linearity and continuity, (18) holds for any $\varphi \in L^{\infty}\left(X_{1}\right) \otimes \ldots \otimes L^{\infty}\left(X_{n}\right)$.

Now assume that $n$ is odd. Let $\xi \in H_{1}, \eta \in H_{n}$ and $\psi_{0,1}: L^{2}\left(X_{1}\right) \rightarrow$ $\mathcal{C}_{2}\left(\mathbb{C}, L^{2}\left(X_{1}\right)\right)$ be the natural identification. We have that $\left(S_{\varphi}\left(f_{1} \otimes \cdots \otimes f_{n-1}\right) \xi, \eta\right)$ coincides with

$$
\left(\check{S}_{(\mathrm{id} \otimes \pi)(1 \otimes \varphi)}\left(\psi_{0,1} \otimes \cdots \otimes \psi_{n-1, n}\right)\left((1 \otimes \xi) \otimes f_{1} \otimes \cdots \otimes f_{n-1}\right), \eta\right)
$$

whenever $\varphi \in L^{\infty}\left(X_{1}\right) \otimes \ldots \otimes L^{\infty}\left(X_{n}\right)$ is an elementary tensor. By linearity and continuity, we have that $\psi_{1, n}\left(S_{\varphi}\left(f_{1} \otimes \cdots \otimes f_{n-1}\right)\right)$ is equal to

$$
\check{S}_{\pi(\varphi)}\left(\psi_{1,2} \otimes \cdots \otimes \psi_{n-1, n}\right)\left(f_{1} \otimes \cdots \otimes f_{n-1}\right)
$$

for all $\varphi \in L^{\infty}\left(X_{1}\right) \otimes \ldots \otimes L^{\infty}\left(X_{n}\right)$. In particular, $S_{\pi(\varphi)}$ takes values in $\mathcal{C}_{2}\left(H_{1}, H_{n}\right)$. As before, it follows that

$$
\begin{aligned}
& \psi_{1, n}^{-1}\left(\check{S}_{\pi(\varphi)}\left(\psi_{1,2} \otimes \ldots \otimes \psi_{n-1, n}\right)\left(f_{1} \otimes \ldots \otimes f_{n-1}\right)\right)\left(x_{1}, x_{n}\right) \\
& \left.=S_{\varphi}\left(f_{1} \otimes \ldots \otimes f_{n-1}\right)\right)\left(x_{1}, x_{n}\right)
\end{aligned}
$$

for every $\varphi \in L^{\infty}\left(X_{1}\right) \otimes \ldots \otimes L^{\infty}\left(X_{n}\right)$. We have thus shown the following.

Proposition 4.6. An element $\varphi \in L^{\infty}\left(X_{1}\right) \otimes \ldots \otimes L^{\infty}\left(X_{n}\right)$ is a Schur multiplier if and only if $\varphi \in \mathbf{M}_{\pi_{1}, \ldots, \pi_{n}}\left(L^{\infty}\left(X_{1}\right), \ldots, L^{\infty}\left(X_{n}\right)\right)$.

Next we want to give a generalisation of Lemma 4.2 for the case where $\varphi$ is a sum of elementary tensors. Let $V, V_{1}, \ldots, V_{n}$ be vector spaces, $L\left(V_{1}, V_{2}\right)$ be the space of all linear mappings from $V_{1}$ into $V_{2}$ and $L(V)=L(V, V)$. Recall that if $f: V_{1} \rightarrow V_{2}$ is a linear map, we let $f_{k, l}: M_{k, l}\left(V_{1}\right) \rightarrow M_{k, l}\left(V_{2}\right)$ be the mapping given by $f_{k, l}\left(\left(v_{i j}\right)\right)=\left(f\left(v_{i j}\right)\right)$, for each $\left(v_{i j}\right) \in M_{k, l}\left(V_{1}\right)$. For an element $v=$ $\left(v_{i j}\right) \in M_{k, l}(V)$ we denote by $v^{\mathrm{t}}=\left(v_{j i}\right) \in M_{l, k}(V)$ the transpose of $v$. Denote by $d: B(K) \rightarrow B\left(K^{\mathrm{d}}\right)$ the mapping sending $A$ to its dual $A^{\mathrm{d}}$. If $A=\left(A_{i j}\right) \in$ $M_{k, l}(B(K))$ let $A^{\mathrm{d}}=\left(A_{i j}^{\mathrm{d}}\right)$.

We will identify $M_{p, q}\left(\mathcal{C}_{2}\left(K_{1}, K_{2}\right)\right)$ with $\mathcal{C}_{2}\left(K_{1}^{q}, K_{2}^{p}\right)$. If $\xi \in M_{p, q}\left(K_{1} \otimes K_{2}\right)$, then $\theta_{p, q}(\xi) \in M_{p, q}\left(\mathcal{C}_{2}\left(K_{1}^{\mathrm{d}}, K_{2}\right)\right)$; using this identification, we will be considering $\theta_{p, q}(\xi)$ as a Hilbert-Schmidt operator from $K_{1}^{q}$ to $K_{2}^{p}$. If $A \in B\left(K_{1}, K_{2}\right)$, then $A \otimes I_{k} \in$ $B\left(K_{1}^{k}, K_{2}^{k}\right)$ is the $k$-fold ampliation of $A$; under the identification $B\left(K_{1}^{k}, K_{2}^{k}\right)=$ $M_{k}\left(B\left(K_{1}, K_{2}\right)\right)$, the operator $A \otimes I_{k}$ has a $k$ by $k$ diagonal matrix, whose every diagonal entry is $A$. The following lemma is straightforward. 
Lemma 4.7. Let $V_{1}, \ldots, V_{n}$ be vector spaces, $\mathcal{L}_{i} \subseteq L\left(V_{i}, V_{i+1}\right)$ a subspace, $i=$ $1, \ldots, n-1$, and

$$
S:\left(L\left(V_{n}\right) \odot L\left(V_{n-1}\right) \odot \cdots \odot L\left(V_{1}\right)\right) \times\left(\mathcal{L}_{n-1} \odot \cdots \odot \mathcal{L}_{1}\right) \rightarrow L\left(V_{1}, V_{n}\right)
$$

be a mapping satisfying

$$
S\left(a_{n} \otimes \cdots \otimes a_{1}, \lambda_{n-1} \otimes \cdots \otimes \lambda_{1}\right)=a_{n} \lambda_{n-1} a_{n-1} \ldots \lambda_{1} a_{1} .
$$

If $A_{1} \in M_{k_{1}, 1}\left(L\left(V_{1}\right)\right), A_{2} \in M_{k_{2}, k_{1}}\left(L\left(V_{2}\right)\right), \ldots, A_{n} \in M_{1, k_{n-1}}\left(L\left(V_{n}\right)\right)$ and $\Lambda_{1} \in$ $M_{l_{1}, 1}\left(\mathcal{L}_{1}\right), \Lambda_{2} \in M_{l_{2}, l_{1}}\left(\mathcal{L}_{2}\right), \ldots, \Lambda_{n-1} \in M_{1, l_{n-2}}\left(\mathcal{L}_{n-1}\right)$, then

$$
S\left(A_{n} \odot \cdots \odot A_{1}, \Lambda_{n-1} \odot \cdots \odot \Lambda_{1}\right)=A_{n} \ldots\left(\Lambda_{2} \otimes I_{k_{2}}\right)\left(A_{2} \otimes I_{l_{1}}\right)\left(\Lambda_{1} \otimes I_{k_{1}}\right) A_{1} .
$$

Lemma 4.8. Let $A_{1} \in M_{1, k_{1}}\left(\mathcal{B}\left(H_{1}\right)\right), A_{2} \in M_{k_{1}, k_{2}}\left(\mathcal{B}\left(H_{2}\right)\right), \ldots, A_{n} \in$ $M_{k_{n-1}, 1}\left(\mathcal{B}\left(H_{n}\right)\right)$ and $\varphi=A_{1} \odot A_{2} \odot \cdots \odot A_{n}$.

(i) Assume $n$ is even. Let $\xi_{1,2} \in M_{1, l_{1}}\left(H_{1} \otimes H_{2}\right), \eta_{2,3} \in M_{l_{1}, l_{2}}\left(H_{2}^{\mathrm{d}} \otimes H_{3}^{\mathrm{d}}\right), \ldots$, $\xi_{n-1, n} \in M_{l_{n-2}, 1}\left(H_{n-1} \otimes H_{n}\right)$ and

$$
\zeta=\xi_{1,2} \odot \eta_{2,3} \odot \cdots \odot \xi_{n-1, n} \in \Gamma\left(H_{1}, \ldots, H_{n}\right) .
$$

Then

$$
S_{\varphi}(\zeta)=A_{n}^{\mathrm{t}} \ldots\left(A_{3}^{\mathrm{t}, \mathrm{d}} \otimes I_{l_{2}}\right)\left(\theta_{l_{1}, l_{2}}\left(\eta_{2,3}\right)^{\mathrm{t}} \otimes I_{k_{2}}\right)\left(A_{2}^{\mathrm{t}, \mathrm{d}} \otimes I_{l_{1}}\right)\left(\theta_{1, l_{1}}\left(\xi_{1,2}\right)^{\mathrm{t}} \otimes I_{k_{1}}\right) A_{1}^{\mathrm{t}, \mathrm{d}} .
$$

(ii) Assume $n$ is odd. Let $\eta_{1,2} \in M_{1, l_{1}}\left(H_{1}^{\mathrm{d}} \otimes H_{2}^{\mathrm{d}}\right), \xi_{2,3} \in M_{l_{1}, l_{2}}\left(H_{2} \otimes H_{3}\right), \ldots$, $\xi_{n-1, n} \in M_{l_{n-2}, 1}\left(H_{n-1} \otimes H_{n}\right)$ and

$$
\zeta=\eta_{1,2} \odot \xi_{2,3} \odot \cdots \odot \xi_{n-1, n} \in \Gamma\left(H_{1}, \ldots, H_{n}\right) .
$$

Then

$$
S_{\varphi}(\zeta)=A_{n}^{\mathrm{t}} \ldots\left(A_{3}^{\mathrm{t}} \otimes I_{l_{2}}\right)\left(\theta_{l_{1}, l_{2}}\left(\xi_{2,3}\right)^{\mathrm{t}} \otimes I_{k_{2}}\right)\left(A_{2}^{\mathrm{t}, \mathrm{d}} \otimes I_{l_{1}}\right)\left(\theta_{1, l_{1}}\left(\eta_{1,2}\right)^{\mathrm{t}} \otimes I_{k_{1}}\right) A_{1}^{\mathrm{t}} .
$$

Proof. Let $f: V_{1} \odot \cdots \odot V_{n} \rightarrow V_{n} \odot \cdots \odot V_{1}$ be the flip, namely the map given on elementary tensors by $f\left(v_{1} \otimes \cdots \otimes v_{n}\right)=v_{n} \otimes \cdots \otimes v_{1}$. Note that if $A_{1} \in M_{1, k_{1}}\left(V_{1}\right)$, $A_{2} \in M_{k_{1}, k_{2}}\left(V_{2}\right), \ldots, A_{n} \in M_{k_{n-1}, 1}\left(V_{n}\right)$, then

$$
f\left(A_{1} \odot \cdots \odot A_{n}\right)=A_{n}^{\mathrm{t}} \odot \cdots \odot A_{1}^{\mathrm{t}} .
$$

Let

$$
D: B\left(H_{1}\right) \odot B\left(H_{2}\right) \odot \cdots \odot B\left(H_{n}\right) \longrightarrow B\left(H_{n}\right) \odot B\left(H_{n-1}^{\mathrm{d}}\right) \odot \cdots \odot B\left(H_{1}^{\mathrm{d}}\right)
$$

be the map

We have that

$$
D=f \circ(d \otimes \mathrm{id} \otimes d \otimes \cdots \otimes \mathrm{id}) .
$$

$$
D(A)=A_{n}^{\mathrm{t}} \odot A_{n-1}^{\mathrm{t}, \mathrm{d}} \odot \cdots \odot A_{1}^{\mathrm{t}, \mathrm{d}} .
$$

Define a mapping $S$ from

$$
\left(B\left(H_{n}\right) \odot B\left(H_{n-1}^{\mathrm{d}}\right) \odot \cdots \odot B\left(H_{1}^{\mathrm{d}}\right)\right) \times\left(\mathcal{C}_{2}\left(H_{n-1}^{\mathrm{d}}, H_{n}\right) \odot \cdots \odot \mathcal{C}_{2}\left(H_{1}^{\mathrm{d}}, H_{2}\right)\right)
$$

into $\mathcal{C}_{2}\left(H_{1}^{\mathrm{d}}, H_{n}\right)$ by

where

$$
S\left(\psi, \zeta^{\prime}\right)=S_{D^{-1}(\psi)}\left(\tilde{\theta}^{-1}\left(\zeta^{\prime}\right)\right)
$$

$$
\tilde{\theta}: \Gamma\left(H_{1}, \ldots, H_{n}\right) \rightarrow \mathcal{C}_{2}\left(H_{n-1}^{\mathrm{d}}, H_{n}\right) \odot \cdots \odot \mathcal{C}_{2}\left(H_{1}^{\mathrm{d}}, H_{2}\right)
$$

is given on elementary tensors by

$$
\tilde{\theta}\left(\xi_{1,2} \otimes \eta_{2,3} \otimes \cdots \otimes \xi_{n-1, n}\right)=\theta\left(\xi_{n-1, n}\right) \otimes \cdots \otimes \theta\left(\eta_{2,3}\right) \otimes \theta\left(\xi_{1,2}\right) .
$$


By Lemma 4.2 (i), the mapping $S$ satisfies the requirements of Lemma 4.7 and

$$
S_{\varphi}(\zeta)=S\left(A_{n}^{\mathrm{t}} \odot A_{n-1}^{\mathrm{t}, \mathrm{d}} \odot \cdots \odot A_{1}^{\mathrm{t}, \mathrm{d}}, \theta_{l_{n-2}, 1}\left(\xi_{n-1, n}\right)^{\mathrm{t}} \odot \cdots \odot \theta_{1, l_{1}}\left(\xi_{1,2}\right)^{\mathrm{t}}\right) .
$$

The claim now follows from Lemma 4.7

The proof of (ii) is similar.

\section{Multipliers For tensor PROduCts of REPRESENTAtions}

It was proved in 21] that the space of all $(\pi, \rho)$-multipliers does not change if the representations $\pi$ and $\rho$ are replaced by approximately equivalent representations. In this section we will prove a corresponding result for multidimensional multipliers. We first recall the notion of approximate equivalence and approximate subordination introduced by Voiculescu in [32.

Let $\pi$ and $\pi^{\prime}$ be $*$-representations of a $C^{*}$-algebra $\mathcal{A}$ on Hilbert spaces $H$ and $H^{\prime}$, respectively. We say that $\pi^{\prime}$ is approximately subordinate to $\pi$ and write $\pi^{\prime} \stackrel{a}{\ll} \pi$ if there is a net $\left\{U_{\lambda}\right\}$ of isometries from $H^{\prime}$ to $H$ such that

$$
\left\|\pi(a) U_{\lambda}-U_{\lambda} \pi^{\prime}(a)\right\| \rightarrow 0 \text { for all } a \in \mathcal{A} .
$$

The representations $\pi^{\prime}$ and $\pi$ are said to be approximately equivalent if the operators $U_{\lambda}$ can be chosen to be unitary; in this case we write $\pi^{\prime} \stackrel{a}{\sim} \pi$.

For $C^{*}$-algebras $\mathcal{A}_{1}, \ldots, \mathcal{A}_{n}$ and the corresponding representations $\pi_{1}, \ldots, \pi_{n}$, we will denote the collection of all $\left(\pi_{1}, \ldots, \pi_{n}\right)$-multipliers in $\mathcal{A}_{1} \otimes \cdots \otimes \mathcal{A}_{n}$ simply by $\mathbf{M}_{\pi_{1}, \ldots, \pi_{n}}$, in case there is no danger of confusion.

Theorem 5.1. Let $\mathcal{A}_{1}, \ldots, \mathcal{A}_{n}$ be $C^{*}$-algebras and $\pi_{i}$ and $\pi_{i}^{\prime}$ be representations of $\mathcal{A}_{i}$ on the Hilbert spaces $H_{i}$ and $H_{i}^{\prime}$, respectively, $i=1, \ldots, n$.

(i) If $\pi_{i}^{\prime} \stackrel{a}{\ll} \pi_{i}, i=1, \ldots, n$, then

$$
\mathbf{M}_{\pi_{1}, \ldots, \pi_{n}} \subseteq \mathbf{M}_{\pi_{1}^{\prime}, \ldots, \pi_{n}^{\prime}} \text { and }\|\varphi\|_{\pi_{1}^{\prime}, \ldots, \pi_{n}^{\prime}} \leq\|\varphi\|_{\pi_{1}, \ldots, \pi_{n}} \text {, for } \varphi \in \mathbf{M}_{\pi_{1}, \ldots, \pi_{n}} .
$$

(ii) If $\pi_{i}^{\prime} \stackrel{a}{\sim} \pi_{i}, i=1, \ldots, n$, then

$$
\mathbf{M}_{\pi_{1}, \ldots, \pi_{n}}=\mathbf{M}_{\pi_{1}^{\prime}, \ldots, \pi_{n}^{\prime}} \text { and }\|\varphi\|_{\pi_{1}, \ldots, \pi_{n}}=\|\varphi\|_{\pi_{1}^{\prime}, \ldots, \pi_{n}^{\prime}}, \text { for } \varphi \in \mathbf{M}_{\pi_{1}, \ldots, \pi_{n}} .
$$

Proof. (i) First let $n$ be even and $\left\{U_{\lambda_{i}}\right\}$ be nets of isometries from $H_{i}^{\prime}$ into $H_{i}$ satisfying

$$
\left\|\pi_{i}\left(a_{i}\right) U_{\lambda_{i}}-U_{\lambda_{i}} \pi_{i}^{\prime}\left(a_{i}\right)\right\| \rightarrow 0, \text { for all } a_{i} \in \mathcal{A}_{i} .
$$

Set $\pi=\bigotimes_{i=1}^{n} \pi_{i}, \pi^{\prime}=\bigotimes_{i=1}^{n} \pi_{i}^{\prime}, \lambda=\left(\lambda_{1}, \ldots, \lambda_{n}\right)$ and $W_{\lambda}=U_{\lambda_{1}} \otimes \ldots \otimes U_{\lambda_{n}}$. Then $W_{\lambda}$ are isometries from $\bigotimes_{i=1}^{n} H_{i}^{\prime}$ to $\bigotimes_{i=1}^{n} H_{n}$ and, for $x \in \mathcal{A}_{1} \odot \ldots \odot \mathcal{A}_{n}$, we have

$$
\left\|\pi(x) W_{\lambda}-W_{\lambda} \pi^{\prime}(x)\right\| \longrightarrow 0 .
$$

As $\left\|W_{\lambda}\right\|=1$ for all $\lambda$, this holds for all $x \in \mathcal{A}_{1} \otimes \ldots \otimes \mathcal{A}_{n}$. By Lemma 4.2 (i) we have that, for any $\xi \in \bigotimes_{i=1}^{n} H_{i}$,

$$
\begin{aligned}
& \theta\left(W_{\lambda}^{*} \xi\right)\left(\theta\left(\eta_{2,3}^{\mathrm{d}}\right)\right) \ldots\left(\theta\left(\eta_{n-2, n-1}^{\mathrm{d}}\right)\right) \\
& =U_{\lambda_{n}}^{*} \theta(\xi)\left(\theta\left(\left(W_{\lambda_{2}, \lambda_{3}} \eta_{2,3}\right)^{\mathrm{d}}\right)\right) \ldots\left(\theta\left(\left(W_{\lambda_{n-2}, \lambda_{n-1}} \eta_{n-2, n-1}\right)^{\mathrm{d}}\right)\right)\left(U_{\lambda_{1}}^{*}\right)^{\mathrm{d}},
\end{aligned}
$$

where $W_{\lambda_{k}, \lambda_{k+1}}=U_{\lambda_{k}} \otimes U_{\lambda_{k+1}}$. Therefore, if $\zeta=\xi_{1,2} \otimes\left(\eta_{2,3}\right)^{\mathrm{d}} \otimes \ldots \otimes \xi_{n-1, n}$, then

$$
\begin{aligned}
& S_{W_{\lambda}^{*} \pi(\varphi) W_{\lambda}}(\zeta) \\
& =U_{\lambda_{n}}^{*} S_{\pi(\varphi)}\left(W_{\lambda_{1}, \lambda_{2}} \xi_{1,2} \otimes\left(W_{\lambda_{2}, \lambda_{3}} \eta_{2,3}\right)^{\mathrm{d}} \otimes \ldots \otimes W_{\lambda_{n-1}, \lambda_{n}} \xi_{n-1, n}\right)\left(U_{\lambda_{1}}^{*}\right)^{\mathrm{d}} .
\end{aligned}
$$


Let $\Gamma_{\lambda}: \Gamma\left(H_{1}^{\prime}, \ldots, H_{n}^{\prime}\right) \rightarrow \Gamma\left(H_{1}, \ldots, H_{n}\right)$ be the linear operator defined on elementary tensors by

$\Gamma_{\lambda}\left(\xi_{1,2} \otimes \eta_{2,3}^{\mathrm{d}} \otimes \ldots \otimes \xi_{n-1, n}\right)=W_{\lambda_{1}, \lambda_{2}} \xi_{1,2} \otimes\left(W_{\lambda_{2}, \lambda_{3}} \eta_{2,3}\right)^{\mathrm{d}} \otimes \ldots \otimes W_{\lambda_{n-1}, \lambda_{n}} \xi_{n-1, n}$.

It follows from (21) and Remark 4.3 that if $\varphi \in \mathbf{M}_{\pi_{1}, \ldots, \pi_{n}}$ and $\zeta \in \Gamma\left(H_{1}^{\prime}, \ldots, H_{n}^{\prime}\right)$, then

$$
\begin{aligned}
\left\|S_{\pi^{\prime}(\varphi)}(\zeta)\right\|_{\mathrm{op}} & \leq\left\|S_{W_{\lambda}^{*} \pi(\varphi) W_{\lambda}}(\zeta)\right\|_{\mathrm{op}}+\left\|S_{W_{\lambda}^{*} \pi(\varphi) W_{\lambda}-\pi^{\prime}(\varphi)}(\zeta)\right\|_{\mathrm{op}} \\
& \leq\left\|S_{\pi(\varphi)}\left(\Gamma_{\lambda} \zeta\right)\right\|_{\mathrm{op}}+\left\|S_{W_{\lambda}^{*} \pi(\varphi) W_{\lambda}-\pi^{\prime}(\varphi)}(\zeta)\right\|_{\mathrm{op}} \\
& \leq\|\varphi\|_{\pi_{1}, \ldots, \pi_{n}}\left\|\Gamma_{\lambda} \zeta\right\|_{\mathrm{h}}+\left\|W_{\lambda}^{*} \pi(\varphi) W_{\lambda}-\pi^{\prime}(\varphi)\right\|_{\mathrm{op}}\|\zeta\|_{2, \wedge} .
\end{aligned}
$$

Since $\left\|W_{\lambda}^{*} \pi(\varphi) W_{\lambda}-\pi^{\prime}(\varphi)\right\|_{\text {op }} \rightarrow 0$, in order to prove that $\varphi \in \mathbf{M}_{\pi_{1}^{\prime}, \ldots, \pi_{n}^{\prime}}$, it suffices to show that $\left\|\Gamma_{\lambda} \zeta\right\|_{\mathrm{h}} \leq\|\zeta\|_{\mathrm{h}}$. If $\xi_{i, i+1} \in H_{i}^{\prime} \otimes H_{i+1}^{\prime}$, then $\theta\left(W_{\lambda_{i}, \lambda_{i+1}} \xi_{i, i+1}\right)=$ $U_{\lambda_{i+1}} \theta\left(\xi_{i, i+1}\right) U_{\lambda_{i}}^{\mathrm{d}}$. Let $\zeta \in \Gamma\left(H_{1}^{\prime}, \ldots, H_{n}^{\prime}\right)$ be of the form

$$
\zeta=\xi_{1,2} \otimes \eta_{2,3}^{\mathrm{d}} \otimes \ldots \otimes \xi_{n-1, n}
$$

where $\xi_{1,2} \in M_{1, k_{2}}\left(H_{1}^{\prime} \otimes H_{2}^{\prime}\right), \eta_{2,3}^{\mathrm{d}} \in M_{k_{2}, k_{3}}\left(\left(H_{2}^{\prime}\right)^{\mathrm{d}} \otimes\left(H_{3}^{\prime}\right)^{\mathrm{d}}\right), \ldots$, and $\xi_{n-1, n} \in$ $M_{k_{n-1}, 1}\left(H_{n-1}^{\prime} \otimes H_{n}^{\prime}\right)$ are such that

$$
\|\zeta\|_{\mathrm{h}}=\left\|\theta_{1, k_{2}}\left(\xi_{1,2}\right)^{\mathrm{t}}\right\|_{\mathrm{op}}\left\|\theta_{k_{2}, k_{3}}\left(\eta_{2,3}^{\mathrm{d}}\right)^{\mathrm{t}}\right\|_{\mathrm{op}} \ldots\left\|\theta_{k_{n-1}, 1}\left(\xi_{n-1, n}\right)^{\mathrm{t}}\right\|_{\mathrm{op}} .
$$

Then

$$
\Gamma_{\lambda} \zeta=W_{\lambda_{1}, \lambda_{2}} \xi_{1,2} \odot\left(W_{\lambda_{2}, \lambda_{3}}^{*, \mathrm{~d}} \otimes I_{k_{2}}\right) \eta_{2,3}^{\mathrm{d}} \odot \ldots \odot\left(W_{\lambda_{n-1}, \lambda_{n}} \otimes I_{k_{n-1}}\right) \xi_{n-1, n},
$$

and as

$$
\begin{aligned}
& \theta_{1, k_{2}}\left(W_{\lambda_{1}, \lambda_{2}} \xi_{1,2}\right)=U_{\lambda_{2}} \theta_{1, k_{2}}\left(\xi_{1,2}\right)\left(U_{\lambda_{1}}^{\mathrm{d}} \otimes I_{k_{2}}\right), \\
& \theta_{k_{2}, k_{3}}\left(\left(\left(W_{\lambda_{2}, \lambda_{3}}^{*}\right)^{\mathrm{d}} \otimes I_{k_{2}}\right) \eta_{2,3}^{\mathrm{d}}\right)=\left(U_{\lambda_{3}}^{\mathrm{d}} \otimes I_{k_{2}}\right) \theta_{2,3}\left(\eta_{2,3}^{\mathrm{d}}\right)\left(U_{\lambda_{2}} \otimes I_{k_{3}}\right), \\
& \theta_{k_{n-1}, 1}\left(\left(W_{\lambda_{n-1}, \lambda_{n}} \otimes I_{k_{n-1}}\right) \xi_{n-1, n}\right)=\left(U_{\lambda_{n}} \otimes I_{k_{n-1}}\right) \theta_{k_{n-1}, 1}\left(\xi_{n-1, n}\right) U_{\lambda_{n-1}}^{\mathrm{d}},
\end{aligned}
$$

we get

$$
\begin{aligned}
\left\|\Gamma_{\lambda} \zeta\right\|_{\mathrm{h}} & \leq\left\|U_{\lambda_{2}} \otimes I_{k_{2}}\right\|_{\mathrm{op}}\left\|\theta_{1, k_{2}}\left(\xi_{1,2}\right)^{\mathrm{t}}\right\|_{\mathrm{op}}\left\|U_{\lambda_{1}}^{\mathrm{d}}\right\|_{\mathrm{op}} \cdots \\
& \ldots\left\|\theta_{k_{n-1}, 1}\left(\xi_{n-1, n}\right)^{\mathrm{t}}\right\|_{\mathrm{op}}\left\|U_{\lambda_{n-1}}^{\mathrm{d}} \otimes I_{k_{n-1}}\right\|_{\mathrm{op}} \\
& =\left\|\theta_{1, k_{2}}\left(\xi_{1,2}\right)^{\mathrm{t}}\right\|_{\mathrm{op}} \cdots\left\|\theta_{k_{n-1}, 1}\left(\xi_{n-1, n}\right)^{\mathrm{t}}\right\|_{\mathrm{op}}=\|\zeta\|_{\mathrm{h}} .
\end{aligned}
$$

This completes the proof for the case where $n$ is even. Now assume that $n$ is odd and let $\Gamma_{\lambda}: \Gamma\left(H_{1}^{\prime}, \ldots, H_{n}^{\prime}\right) \rightarrow \Gamma\left(H_{1}, \ldots, H_{n}\right)$ be the linear operator defined on elementary tensors by

$$
\Gamma_{\lambda}\left(\xi_{1,2}^{\mathrm{d}} \otimes \ldots \otimes \eta_{n-1, n}\right)=\left(W_{\lambda_{1}, \lambda_{2}} \xi_{1,2}\right)^{\mathrm{d}} \otimes \ldots \otimes W_{\lambda_{n-1}, \lambda_{n}} \eta_{n-1, n} .
$$

An estimate similar to the above shows again that $\left\|\Gamma_{\lambda} \zeta\right\|_{\mathrm{h}} \leq\|\zeta\|_{\mathrm{h}}$. 
By the definition of the map $S_{\pi^{\prime}(\varphi)}$ and the arguments above, we obtain

$$
\begin{aligned}
& \left\|S_{\pi^{\prime}(\varphi)}(\zeta)\right\|_{\mathrm{op}} \leq\left\|S_{W_{\lambda}^{*} \pi(\varphi) W_{\lambda}}(\zeta)\right\|_{\mathrm{op}}+\left\|S_{\left(W_{\lambda}^{*} \pi(\varphi) W_{\lambda}-\pi^{\prime}(\varphi)\right)}(\zeta)\right\|_{\mathrm{op}} \\
& =\sup _{\xi_{1} \in H_{1}^{\prime},\left\|\xi_{1}\right\|=1}\left\|S_{1 \otimes W_{\lambda}^{*} \pi(\varphi) W_{\lambda}}\left(\xi_{1} \otimes \zeta\right)\right\|_{H_{n}^{\prime}}+\left\|S_{\left(W_{\lambda}^{*} \pi(\varphi) W_{\lambda}-\pi^{\prime}(\varphi)\right)}(\zeta)\right\|_{\mathrm{op}} \\
& \leq \sup _{\xi_{1} \in H_{1}^{\prime},\left\|\xi_{1}\right\|=1}\left\|S_{1 \otimes \pi(\varphi)}\left(U_{\lambda_{1}} \xi_{1} \otimes \Gamma_{\lambda} \zeta\right)\right\|_{H_{n}}+\left\|S_{\left(W_{\lambda}^{*} \pi(\varphi) W_{\lambda}-\pi^{\prime}(\varphi)\right)}(\zeta)\right\|_{\mathrm{op}} \\
& \leq \sup _{\eta_{1} \in H_{1},\left\|\eta_{1}\right\|=1}\left\|S_{1 \otimes \pi(\varphi)}\left(\eta_{1} \otimes \Gamma_{\lambda} \zeta\right)\right\|_{H_{n}}+\left\|W_{\lambda}^{*} \pi(\varphi) W_{\lambda}-\pi^{\prime}(\varphi)\right\|_{\mathrm{op}}\|\zeta\|_{2, \wedge} \\
& =\left\|S_{\pi(\varphi)}\left(\Gamma_{\lambda} \zeta\right)\right\|_{\mathrm{op}}+\left\|W_{\lambda}^{*} \pi(\varphi) W_{\lambda}-\pi^{\prime}(\varphi)\right\|_{\mathrm{op}}\|\zeta\|_{2, \wedge} \\
& \leq\|\varphi\|_{\pi_{1}, \ldots, \pi_{n}}\|\| \Gamma_{\lambda} \zeta\left\|_{\mathrm{h}}+\right\| W_{\lambda}^{*} \pi(\varphi) W_{\lambda}-\pi^{\prime}(\varphi)\left\|_{\mathrm{op}}\right\| \zeta \|_{2, \wedge} \\
& \leq\|\varphi\|_{\pi_{1}, \ldots, \pi_{n}}\|\| \zeta\left\|_{\mathrm{h}}+\right\| W_{\lambda}^{*} \pi(\varphi) W_{\lambda}-\pi^{\prime}(\varphi)\left\|_{\mathrm{op}}\right\| \zeta \|_{2, \wedge} .
\end{aligned}
$$

As $\left\|W_{\lambda}^{*} \pi(\varphi) W_{\lambda}-\pi^{\prime}(\varphi)\right\|_{\text {op }} \rightarrow 0$ we obtain the desired statement.

(ii) is a direct consequence of (i).

For $T \in B(H)$, set $\operatorname{rank}(T)=\overline{\operatorname{dim}(T H)}$. It was proved in [17, Theorem 5.1] that for $*$-representations $\pi$ and $\pi^{\prime}$ of a $C^{*}$-algebra $\mathcal{A}$,

$$
\pi^{\prime} \stackrel{a}{\ll} \pi \Longleftrightarrow \operatorname{rank}\left(\pi^{\prime}(a)\right) \leq \operatorname{rank}(\pi(a)) \text { for each } a \in \mathcal{A} .
$$

The next statement is a multidimensional version of [21, Corollory 5.3]. Its proof follows the lines of the proof of the corresponding statement in the two-dimensional case and uses Theorem 5.1 instead of [21, Theorem 5.2].

Corollary 5.2. Let $\pi_{i}, \pi_{i}^{\prime}$ be representations of separable $C^{*}$-algebras $\mathcal{A}_{i}, i=$ $1, \ldots, n$. Assume that

$$
\min \left\{\aleph_{0}, \operatorname{rank}\left(\pi_{i}^{\prime}\left(a_{i}\right)\right)\right\} \leq \min \left\{\aleph_{0}, \operatorname{rank}\left(\pi_{i}\left(a_{i}\right)\right)\right\},
$$

for each $a_{i} \in \mathcal{A}_{i}$ and $i=1, \ldots, n$.

Then $\mathbf{M}_{\pi_{1}, \ldots, \pi_{n}} \subseteq \mathbf{M}_{\pi_{1}^{\prime}, \ldots, \pi_{n}^{\prime}}$ and $\|\varphi\|_{\pi_{1}^{\prime}, \ldots, \pi_{n}^{\prime}} \leq\|\varphi\|_{\pi_{1}, \ldots, \pi_{n}}$ for $\varphi \in \mathbf{M}_{\pi_{1}, \ldots, \pi_{n}}$.

Recall that a $*$-representation $\pi$ of a $C^{*}$-algebra $\mathcal{A}$ has a separating vector if there is a cyclic vector for the commutant $\pi(\mathcal{A})^{\prime}$.

Lemma 5.3. Let $\mathcal{H}, H_{1}, \ldots, H_{n}$ be Hilbert spaces, $\pi_{1}, \ldots, \pi_{n}$ be representations of the $C^{*}$-algebras $\mathcal{A}_{1}, \ldots, \mathcal{A}_{n}$ on $H_{1}, \ldots, H_{n}$ and $\pi_{i} \otimes 1$ be the ampliation of $\pi_{i}$ on $H_{i} \otimes \mathcal{H}$, respectively. Assume that $\pi_{1}$ and $\pi_{n}$ have separating vectors. Then

$$
\mathbf{M}_{\pi_{1}, \ldots, \pi_{n}}=\mathbf{M}_{\pi_{1} \otimes 1, \ldots, \pi_{n} \otimes 1}
$$

and the multiplier norms on these spaces coincide.

Proof. We use ideas from the proofs of [28, Theorem 2.1] and Lemma 3.3. For simplicity we assume that $n=3$ and that $\mathcal{H}$ is separable. Let $\varphi \in \mathbf{M}_{\pi_{1}, \pi_{2}, \pi_{3}}$ with $\|\varphi\|_{\pi_{1}, \pi_{2}, \pi_{3}}=1$ and set $S=S_{\left(\pi_{1} \otimes 1\right) \otimes\left(\pi_{2} \otimes 1\right) \otimes\left(\pi_{3} \otimes 1\right)(\varphi)}$. The mapping $S$ can be regarded as a mapping on

$$
\mathcal{C}_{2}\left(\left(H_{2} \otimes \mathcal{H}\right)^{\mathrm{d}}, H_{3} \otimes \mathcal{H}\right) \odot \mathcal{C}_{2}\left(H_{1} \otimes \mathcal{H},\left(H_{2} \otimes \mathcal{H}\right)^{\mathrm{d}}\right)
$$

by setting $S\left(\theta\left(\xi_{2,3}\right) \otimes \theta\left(\eta_{1,2}^{\mathrm{d}}\right)\right)=S\left(\eta_{1,2}^{\mathrm{d}} \otimes \xi_{2,3}\right)$ for $\zeta=\eta_{1,2}^{\mathrm{d}} \otimes \xi_{2,3} \in \Gamma\left(H_{1} \otimes \mathcal{H}, H_{2} \otimes\right.$ $\left.\mathcal{H}, H_{3} \otimes \mathcal{H}\right)$. Similarly, the mapping $S_{\pi_{1} \otimes \pi_{2} \otimes \pi_{3}(\varphi)}$ can be regarded as a mapping on $\mathcal{C}_{2}\left(H_{2}^{\mathrm{d}}, H_{3}\right) \odot \mathcal{C}_{2}\left(H_{1}, H_{2}^{\mathrm{d}}\right)$. It follows from Lemma 4.8 that $S_{\pi_{1} \otimes \pi_{2} \otimes \pi_{3}(\varphi)}$ is $\left(\pi_{3}\left(\mathcal{A}_{3}\right)^{\prime},\left(\pi_{2}\left(\mathcal{A}_{2}\right)^{\prime}\right)^{\mathrm{d}}, \pi_{1}\left(\mathcal{A}_{1}\right)^{\prime}\right)$-modular. 
Assume that $\|\varphi\|_{\pi_{1} \otimes 1, \pi_{2} \otimes 1, \pi_{3} \otimes 1}>1$. Then there exists an element $T=$ $\left(T_{1}^{2}, \ldots, T_{s}^{2}\right) \odot\left(T_{1}^{1}, \ldots, T_{s}^{1}\right)^{\mathrm{t}}$ in the space defined in (23) with

$$
\left\|\sum\left(T_{i}^{1}\right)^{*} T_{i}^{1}\right\|\left\|\sum T_{i}^{2}\left(T_{i}^{2}\right)^{*}\right\|=1,
$$

and vectors $\xi_{0} \in H_{1} \otimes \mathcal{H}, \eta_{0} \in H_{3} \otimes \mathcal{H}$ of norm less than one such that

$$
\left|\left(S(T) \xi_{0}, \eta_{0}\right)\right|>1 .
$$

Fix a basis $\left\{f_{l}\right\}$ of $\mathcal{H}$ and denote by $P_{n}$ the projection onto the space generated by the first $n$ vectors in this basis. Then, as

$$
\left(1_{H_{3}} \otimes P_{n}\right) S(T)\left(1_{H_{1}} \otimes P_{n}\right) \rightarrow S(T)
$$

weakly, there exists $n \geq 1$ such that

$$
\left|\left(\left(1_{H_{3}} \otimes P_{n}\right) S(T)\left(1_{H_{1}} \otimes P_{n}\right) \xi_{0}, \eta_{0}\right)\right|>1 .
$$

Thus we may assume that $\xi_{0} \in H_{1} \otimes P_{n} \mathcal{H}$ and $\eta_{0} \in H_{3} \otimes P_{n} \mathcal{H}$, say

$$
\xi_{0}=\left(\xi_{1}, \ldots, \xi_{n}, 0, \ldots\right), \eta_{0}=\left(\eta_{1}, \ldots, \eta_{n}, 0 \ldots\right) .
$$

As $\pi_{1}\left(\mathcal{A}_{1}\right)^{\prime}$ and $\pi_{3}\left(\mathcal{A}_{3}\right)^{\prime}$ have cyclic vectors, say $\xi$ and $\eta$ respectively, we may assume that $\xi_{i}=a_{i} \xi, \eta_{i}=b_{i} \eta$ for some $a_{i} \in \pi_{1}\left(\mathcal{A}_{1}\right)^{\prime}$ and $b_{i} \in \pi_{3}\left(\mathcal{A}_{3}\right)^{\prime}$. Let $a=\sum a_{i}^{*} a_{i}, b=\sum b_{i}^{*} b_{i}$. Assuming first that $a, b$ are invertible we set $\tilde{a}_{i}=$ $a_{i} a^{-1 / 2}, \tilde{b}_{i}=b_{i} b^{-1 / 2}$. Then for $\tilde{\xi}=a^{1 / 2} \xi, \tilde{\eta}=b^{1 / 2} \eta$ we have $\xi_{i}=\tilde{a}_{i} \tilde{\xi}$ and $\eta_{i}=\tilde{b}_{i} \tilde{\eta}$. We write $T_{i}^{k}=\left(\left(T_{i}^{k}\right)_{l m}\right)$, where $\left(T_{i}^{1}\right)_{l m}=\left(1_{H_{2}^{\mathrm{d}}} \otimes P\left(f_{l}^{\mathrm{d}}\right)\right) T_{i}^{1}\left(1_{H_{1}} \otimes P\left(f_{m}\right)\right)$, $\left(T_{i}^{2}\right)_{l m}=\left(1_{H_{3}} \otimes P\left(f_{l}\right)\right) T_{i}^{2}\left(1_{H_{2}^{\mathrm{d}}} \otimes P\left(f_{m}^{\mathrm{d}}\right)\right)$, where $P(f)$ is the projection onto the one-dimensional space generated by $f$. Using the modularity of $S_{\pi_{1} \otimes \pi_{2} \otimes \pi_{3}(\varphi)}$, we obtain

$$
\begin{aligned}
\left|\left(S(T) \xi_{0}, \eta_{0}\right)\right| & =\left|\sum_{i=1}^{s}\left(S\left(T_{i}^{2} \otimes T_{i}^{1}\right) \xi_{0}, \eta_{0}\right)\right| \\
& =\left|\sum_{i=1}^{s} \sum_{l, m=1}^{n} \sum_{k=1}^{\infty}\left(S_{\pi_{1} \otimes \pi_{2} \otimes \pi_{3}(\varphi)}\left(\left(T_{i}^{2}\right)_{l k} \otimes\left(T_{i}^{1}\right)_{k m}\right) \tilde{a}_{m} \tilde{\xi}, \tilde{b}_{l} \tilde{\eta}\right)\right| \\
& =\left|\sum_{i=1}^{s} \sum_{l, m=1}^{n} \sum_{k=1}^{\infty}\left(S_{\pi_{1} \otimes \pi_{2} \otimes \pi_{3}(\varphi)}\left(\tilde{b}_{l}^{*}\left(T_{i}^{2}\right)_{l k} \otimes\left(T_{i}^{1}\right)_{k m} \tilde{a}_{m}\right) \tilde{\xi}, \tilde{\eta}\right)\right| .
\end{aligned}
$$

The next step is to prove that $\sum_{i=1}^{s} \sum_{k=1}^{\infty}\left(\sum_{l=1}^{n} \tilde{b}_{l}^{*}\left(T_{i}^{2}\right)_{l k}\right) \otimes\left(\sum_{m=1}^{n}\left(T_{i}^{1}\right)_{k m} \tilde{a}_{m}\right)$ belongs to $\mathcal{K}\left(H_{2}^{\mathrm{d}}, H_{3}\right) \otimes_{\mathrm{h}} \mathcal{K}\left(H_{1}, H_{2}^{\mathrm{d}}\right)$. Observe first that the row operator

$$
R_{i}=\left(\sum_{l=1}^{n} \tilde{b}_{l}^{*}\left(T_{i}^{2}\right)_{l 1}, \ldots, \sum_{l=1}^{n} \tilde{b}_{l}^{*}\left(T_{i}^{2}\right)_{l k}, \ldots\right)
$$

is equal to the product of the row operator $\tilde{B}=\left(\tilde{b}_{1}, \ldots, \tilde{b}_{n}, 0, \ldots\right)$ and the HilbertSchmidt operator $T_{i}^{2}$. Set $R=\left(R_{1}, \ldots, R_{s}\right)=\left(\tilde{B} T_{1}^{2}, \ldots, \tilde{B} T_{s}^{2}\right)$. 
As each $T_{i}^{2}$ is the operator norm-limit of the operators $T_{i}^{2}\left(1_{H_{2}^{\mathrm{d}}} \otimes P_{k}\right)$ as $k \rightarrow \infty$, the operator $R_{i}$ is the uniform limit of the sequence of truncated operators $R_{i}^{k}=$ $\left(\sum_{l=1}^{n} \tilde{b}_{l}^{*}\left(T_{i}^{2}\right)_{l 1}, \ldots, \sum_{l=1}^{n} \tilde{b}_{l}^{*}\left(T_{i}^{2}\right)_{l k}, 0 \ldots\right)$. Thus

$$
R R^{*}=\sum_{i=1}^{s} \sum_{k=1}^{\infty}\left(\sum_{l=1}^{n} \tilde{b}_{l}^{*}\left(T_{i}^{2}\right)_{l k}\right)\left(\sum_{l=1}^{n} \tilde{b}_{l}^{*}\left(T_{i}^{2}\right)_{l k}\right)^{*},
$$

where the series converges uniformly and

$$
\begin{array}{r}
\left\|\sum_{i=1}^{s} \sum_{k=1}^{\infty}\left(\sum_{l=1}^{n} \tilde{b}_{l}^{*}\left(T_{i}^{2}\right)_{l k}\right)\left(\sum_{l=1}^{n} \tilde{b}_{l}^{*}\left(T_{i}^{2}\right)_{l k}\right)^{*}\right\|=\left\|R R^{*}\right\|=\left\|\sum_{i=1}^{s} R_{i} R_{i}^{*}\right\| \\
=\left\|\tilde{B}\left(\sum_{i=1}^{s} T_{i}^{2}\left(T_{i}^{2}\right)^{*}\right) \tilde{B}^{*}\right\| \leq\|\tilde{B}\|^{2}\|\| \sum_{i=1}^{s} T_{i}^{2}\left(T_{i}^{2}\right)^{*} \| \leq 1 .
\end{array}
$$

In the same way one shows that the series

$$
\sum_{k=1}^{\infty}\left(\sum_{m=1}^{n}\left(T_{i}^{1}\right)_{k m} \tilde{a}_{m}\right)\left(\sum_{m=1}^{n}\left(T_{i}^{1}\right)_{k m} \tilde{a}_{m}\right)^{*}
$$

converges uniformly and

$$
\left\|\sum_{i=1}^{s} \sum_{k=1}^{\infty}\left(\sum_{m=1}^{n}\left(T_{i}^{1}\right)_{k m} \tilde{a}_{m}\right)\left(\sum_{m=1}^{n}\left(T_{i}^{1}\right)_{k m} \tilde{a}_{m}\right)^{*}\right\| \leq 1 .
$$

Thus $\sum_{i=1}^{s} \sum_{k=1}^{\infty}\left(\sum_{l=1}^{n} \tilde{b}_{l}^{*}\left(T_{i}^{2}\right)_{l k}\right) \otimes\left(\sum_{m=1}^{n}\left(T_{i}^{1}\right)_{k m} \tilde{a}_{m}\right) \in \mathcal{K}\left(H_{1}, H_{2}^{\mathrm{d}}\right) \otimes_{\mathrm{h}} \mathcal{K}\left(H_{2}^{\mathrm{d}}, H_{3}\right)$ and

$$
\left\|\sum_{i=1}^{s} \sum_{k=1}^{\infty}\left(\sum_{l=1}^{n} \tilde{b}_{l}^{*}\left(T_{i}^{2}\right)_{l k}\right) \otimes\left(\sum_{m=1}^{n}\left(T_{i}^{1}\right)_{k m} \tilde{a}_{m}\right)\right\|_{\mathrm{h}} \leq 1 .
$$

Next $\|\tilde{\xi}\|^{2}=\left(b^{1 / 2} \xi, b^{1 / 2} \xi\right)=(b \xi, \xi)=\sum_{i}\left(b_{i} \xi, b_{i} \xi\right)=\left\|\xi_{0}\right\|^{2}<1$. Similarly, $\|\tilde{\eta}\|<1$. Since $\|\varphi\|_{\pi_{1}, \pi_{2}, \pi_{3}}=1$, it now follows from (24) that

$$
\left|\left(S(T) \xi_{0}, \eta_{0}\right)\right| \leq\left\|\sum_{i=1}^{s} \sum_{k=1}^{\infty}\left(\sum_{l=1}^{n} \tilde{b}_{l}^{*}\left(T_{i}^{2}\right)_{l k}\right) \otimes\left(\sum_{m=1}^{n}\left(T_{i}^{1}\right)_{k m} \tilde{a}_{m}\right)\right\|_{\mathrm{h}}\|\tilde{\xi}\|\|\tilde{\eta}\|,
$$

which does not exceed 1, a contradiction.

If $a$ or $b$ is not invertible, let $\epsilon>0$ be such that $\hat{\xi}_{0} \stackrel{\text { def }}{=}\left(\xi_{1}, \ldots, \xi_{n}, \epsilon \xi, 0, \ldots\right)$ and $\hat{\eta}_{0} \stackrel{\text { def }}{=}\left(\eta_{1}, \ldots, \eta_{n}, \epsilon \eta, 0, \ldots\right)$ have norm less than one and $\left|\left(S(T) \hat{\xi}_{0}, \hat{\eta}_{0}\right)\right|>1$. Choose $a_{i}$ and $b_{i}$ in the same way as before, and let $a_{n+1}=\epsilon I, b_{n+1}=\epsilon I, a=\sum_{i=1}^{n+1} a_{i}^{*} a_{i}$ and $b=\sum_{i=1}^{n+1} b_{i}^{*} b_{i}$. Then $a$ and $b$ are invertible and the proof proceeds in the same fashion.

We have proved that $\mathbf{M}_{\pi_{1}, \ldots, \pi_{n}} \subseteq \mathbf{M}_{\pi_{1} \otimes 1, \ldots, \pi_{n} \otimes 1}$ and that $\|\cdot\|_{\pi_{1} \otimes 1, \ldots, \pi_{n} \otimes 1} \leq$ $\|\cdot\|_{\pi_{1}, \ldots, \pi_{n}}$. The converse inequality is easy to show, and thus the proof is complete.

Corollary 5.4. Let $\pi_{i}$ be a representation of the $C^{*}$-algebra $\mathcal{A}_{i}, i=1, \ldots, n$. Assume that $\pi_{1}$ and $\pi_{n}$ have separating vectors. If

$$
\operatorname{ker}\left(\pi_{i}\right) \subseteq \operatorname{ker}\left(\pi_{i}^{\prime}\right), \text { for each } i=1, \ldots, n,
$$

then $\mathbf{M}_{\pi_{1}, \ldots, \pi_{n}} \subseteq \mathbf{M}_{\pi_{1}^{\prime}, \ldots, \pi_{n}^{\prime}}$ and $\|\varphi\|_{\pi_{1}^{\prime}, \ldots, \pi_{n}^{\prime}} \leq\|\varphi\|_{\pi_{1}, \ldots, \pi_{n}}$, for each $\varphi \in \mathbf{M}_{\pi_{1}, \ldots, \pi_{n}}$. 
Proof. The proof is similar to that of [21, Corollary 5.8]; we include it for completeness. Let $\mathcal{H}$ be an infinite-dimensional Hilbert space of sufficiently large dimension. Then (25) implies

$$
\operatorname{rank}\left(\pi_{i}^{\prime}\left(a_{i}\right)\right) \leq \operatorname{rank}\left(\pi_{i}\left(a_{i}\right) \otimes 1\right), \text { for all } a_{i} \in \mathcal{A}_{i} .
$$

By (22), $\pi_{i}^{\prime} \stackrel{a}{\ll} \pi_{i} \otimes 1$. Now applying Theorem 5.1 and then Lemma 5.3 we obtain the statement.

Using Corollary 5.4 and the results from 21] we will now show that if the $C^{*}$ algebras $\mathcal{A}_{i}$ are commutative, then the space $\mathbf{M}_{\pi_{1}, \ldots, \pi_{n}}\left(\mathcal{A}_{1}, \ldots, \mathcal{A}_{n}\right)$ of multipliers depends only on the supports of spectral measures corresponding to the representations $\pi_{i}$.

Assume that $\mathcal{A}_{i}$ is commutative, $i=1, \ldots, n$ and let $X_{i}$ be the maximal ideal space of $\mathcal{A}_{i}$; then $\mathcal{A}_{i} \simeq C_{0}\left(X_{i}\right)$. Let $\pi_{i}$ be a representation of $\mathcal{A}_{i}$ and $\mathcal{E}_{\pi_{i}}$ be the spectral measure on $X_{i}$ corresponding to $\pi_{i}$.

It was proved in [21, Lemma 7.2] that if $f \in C_{0}(X)$ and the representation $\pi$ of $C_{0}(X)$ is such that rank $(\pi(f))<\infty$, then

$$
\operatorname{rank}(\pi(f))=\sum_{x \in S\left(f, \mathcal{E}_{\pi}\right)} \operatorname{dim}\left(\mathcal{E}_{\pi}(\{x\})\right),
$$

where $S\left(f, \mathcal{E}_{\pi}\right)=\left\{x \in \operatorname{supp} \mathcal{E}_{\pi}: f(x) \neq 0\right\}$. Thus the condition

$$
\operatorname{supp} \mathcal{E}_{\pi^{\prime}} \subset \operatorname{supp} \mathcal{E}_{\pi}
$$

implies $\operatorname{ker} \pi(f) \subseteq \operatorname{ker} \pi^{\prime}(f)$. As each representation $\pi$ of a commutative algebra $C_{0}(X)$ has a separating vector we have the following.

Corollary 5.5. Let $\pi_{i}, \pi_{i}^{\prime}$ be separable representations of the $C^{*}$-algebra $\mathcal{A}_{i}=$ $C_{0}\left(X_{i}\right)$ and $\mathcal{E}_{\pi_{i}}$ and $\mathcal{E}_{\pi_{i}^{\prime}}$ be the corresponding spectral measures $(i=1, \ldots, n)$. If

$$
\operatorname{supp} \mathcal{E}_{\pi_{i}^{\prime}} \subseteq \operatorname{supp} \mathcal{E}_{\pi_{i}}, \text { for each } i=1, \ldots, n,
$$

then $\mathbf{M}_{\pi_{1}, \ldots, \pi_{n}} \subseteq \mathbf{M}_{\pi_{1}^{\prime}, \ldots, \pi_{n}^{\prime}}$.

Let $\mu_{i}$ be measures on $X_{i}$. Let $\pi_{i}$ be a representation of $C_{0}\left(X_{i}\right)$ on $L_{2}\left(X_{i}, \mu_{i}\right)$ defined by $\left(\pi_{i}(f) h\right)\left(x_{i}\right)=f\left(x_{i}\right) h\left(x_{i}\right)$. We call $\varphi \in C_{0}\left(X_{1} \times \ldots \times X_{n}\right)$ a $\left(\mu_{1}, \ldots, \mu_{n}\right)$ multiplier if $\varphi \in \mathbf{M}_{\pi_{1}, \ldots, \pi_{n}}$ and let $\|\varphi\|_{\mu_{1}, \ldots, \mu_{n}}=\|\varphi\|_{\pi_{1}, \ldots, \pi_{n}}$.

By Corollary 5.5] the set of all the $\left(\mu_{1}, \ldots, \mu_{n}\right)$-multipliers depends only on the supports of the measures $\mu_{i}$. The next statement shows the connection between $\left(\mu_{1}, \ldots, \mu_{n}\right)$-multipliers and multidimensional Schur multipliers (with respect to discrete measures).

Corollary 5.6. Let $X_{i}$ be locally compact spaces with countable bases and let $\mu_{i}$ be Borel $\sigma$-finite measures on $X_{i}$ with $\operatorname{supp} \mu_{i}=X_{i}$. Then $\varphi \in C_{0}\left(X_{1} \times \ldots \times X_{n}\right)$ is $a\left(\mu_{1}, \ldots, \mu_{n}\right)$-multiplier if and only if $\varphi$ is a Schur multiplier on $X_{1} \times \ldots \times X_{n}$. Moreover, in this case $\|\varphi\|_{\mu_{1}, \ldots, \mu_{n}}=\left\|S_{\varphi}\right\|$.

Proof. The proof is similar to that of [21, Theorem 7.5].

\section{UNIVERSAL MULTIPLIERS}

The main goal of this section is to give a full description of the multipliers which does not depend on the choice of the representations of the $C^{*}$-algebras $\mathcal{A}_{1}, \mathcal{A}_{2}, \ldots, \mathcal{A}_{n}$. Recall that an element $\varphi \in \mathcal{A}_{1} \otimes \ldots \otimes \mathcal{A}_{n}$ is called a universal multiplier if $\varphi$ is a $\left(\pi_{1}, \pi_{2}, \ldots, \pi_{n}\right)$-multiplier for all representations $\pi_{1}, \pi_{2}, \ldots, \pi_{n}$ of 
$\mathcal{A}_{1}, \mathcal{A}_{2}, \ldots, \mathcal{A}_{n}$, respectively. The set of all universal multipliers in $\mathcal{A}_{1} \otimes \cdots \otimes \mathcal{A}_{n}$ is denoted by $\mathbf{M}\left(\mathcal{A}_{1}, \ldots, \mathcal{A}_{n}\right)$.

Along with the universal multipliers, we will describe another class of multipliers, which we call projective universal multipliers and define as follows. Let $H_{1}, \ldots, H_{n}$ be Hilbert spaces. Equip $\Gamma\left(H_{1}, \ldots, H_{n}\right)$ with the projective tensor norm $\|\cdot\|_{\wedge}$, where each of the terms $H_{i} \otimes H_{i+1}$ (resp. $H_{i-1}^{\mathrm{d}} \otimes H_{i}^{\mathrm{d}}$ ) is given its operator norm. We call an element $\varphi \in \mathcal{B}\left(H_{1} \otimes \cdots \otimes H_{n}\right)$ a concrete projective multiplier if there exists $C>0$ such that $\left\|S_{\varphi}(\zeta)\right\|_{\text {op }} \leq C\|\zeta\|_{\wedge}$, for all $\zeta \in \Gamma\left(H_{1}, \ldots, H_{n}\right)$. If $\mathcal{A}_{1}, \ldots \mathcal{A}_{n}$ are $C^{*}$-algebras, an element $\varphi \in \mathcal{A}_{1} \otimes \cdots \otimes \mathcal{A}_{n}$ will be called a projective universal multiplier if $\left(\pi_{1} \otimes \cdots \otimes \pi_{n}\right)(\varphi)$ is a concrete projective multiplier for all choices of the representations $\pi_{1}, \ldots, \pi_{n}$ of $\mathcal{A}_{1}, \ldots, \mathcal{A}_{n}$, respectively. We denote by $\mathbf{M}^{\wedge}\left(\mathcal{A}_{1}, \ldots, \mathcal{A}_{n}\right)$ the set of all projective universal multipliers.

If $\varphi \in \mathbf{M}\left(\mathcal{A}_{1}, \ldots, \mathcal{A}_{n}\right)$ let

$$
\|\varphi\|_{\text {univ }}=\sup _{\pi_{1}, \pi_{2}, \ldots, \pi_{n}}\|\varphi\|_{\pi_{1}, \pi_{2}, \ldots, \pi_{n}} .
$$

Note that $\|\varphi\|_{\text {univ }}$ is finite. In fact, assume that there exist representations $\pi_{1, k}, \ldots$, $\pi_{n, k}$, such that $\|\varphi\|_{\pi_{1, k}, \pi_{2, k}, \ldots, \pi_{n, k}} \rightarrow_{k \rightarrow \infty} \infty$ and let $\pi_{1}=\bigoplus_{k} \pi_{1, k}, \pi_{2}=\bigoplus_{k} \pi_{2, k}, \ldots$, $\pi_{n}=\bigoplus_{k} \pi_{n, k}$. Then, by Theorem 5.1

$$
\|\varphi\|_{\pi_{1, k}, \pi_{2, k}, \ldots, \pi_{n, k}} \leq\|\varphi\|_{\pi_{1}, \pi_{2}, \ldots, \pi_{n}}
$$

for all $k \in \mathbb{N}$, which contradicts the fact that $\varphi \in \mathbf{M}\left(\mathcal{A}_{1}, \ldots, \mathcal{A}_{n}\right)$.

It is clear that $\mathbf{M}\left(\mathcal{A}_{1}, \ldots, \mathcal{A}_{n}\right)$ is a linear subspace of $\mathcal{A}_{1} \otimes \cdots \otimes \mathcal{A}_{n}$ containing $\mathcal{A}_{1} \odot \cdots \odot \mathcal{A}_{n}$.

Recall that the Haagerup norm on $\mathcal{A}_{1} \odot \mathcal{A}_{2} \odot \ldots \odot \mathcal{A}_{n}$ is

$$
\begin{gathered}
\|\omega\|_{\mathrm{h}}=\inf \left\{\left\|\omega_{1}\right\|\left\|\omega_{2}\right\| \ldots\left\|\omega_{n}\right\|: \omega=\omega_{1} \odot \omega_{2} \odot \ldots \odot \omega_{n},\right. \\
\left.\omega_{1} \in M_{1, i_{1}}\left(\mathcal{A}_{1}\right), \omega_{2} \in M_{i_{1}, i_{2}}\left(\mathcal{A}_{2}\right), \ldots, \omega_{n} \in M_{i_{n-1}, 1}\left(\mathcal{A}_{n}\right), i_{1}, \ldots, i_{n-1} \in \mathbb{N}\right\} .
\end{gathered}
$$

A modification of the Haagerup norm on the algebraic tensor product of two $C^{*}$-algebras was considered in [20, 21]. We now introduce a natural generalisation of this norm for arbitrary $n$. Recall the maps $\omega \mapsto \omega^{\mathrm{t}}$ and $\omega \mapsto \omega^{\mathrm{d}}$ on $M_{n}(\mathcal{A})=$ $M_{n}(\mathbb{C}) \otimes \mathcal{A}$ given on elementary tensors by $(a \otimes b)^{\mathrm{t}}=a^{\mathrm{t}} \otimes b$ and $(a \otimes b)^{\mathrm{d}}=a \otimes b^{\mathrm{d}}$ (here $\mathcal{A}$ is a $C^{*}$-subalgebra of $B(H)$ for some Hilbert space $H$ ). We set

$$
\begin{aligned}
& \|\omega\|_{\mathrm{ph}}=\inf \left\{\prod_{0 \leq i<\frac{n}{2}}\left\|\omega_{n-2 i}^{\mathrm{t}}\right\|\left\|\omega_{n-2 i-1}\right\|: \omega=\omega_{1} \odot \omega_{2} \odot \ldots \odot \omega_{n}, \omega_{0}=I,\right. \\
& \left.\omega_{1} \in M_{1, i_{1}}\left(\mathcal{A}_{1}\right), \omega_{2} \in M_{i_{1}, i_{2}}\left(\mathcal{A}_{2}\right), \ldots, \omega_{n} \in M_{i_{n-1}, 1}\left(\mathcal{A}_{n}\right), i_{1}, \ldots, i_{n-1} \in \mathbb{N}\right\},
\end{aligned}
$$

In the case $n=2$, the above norm was denoted in 20] by $\|\cdot\|_{\mathrm{h}^{\prime}}$. Clearly, if the algebras $\mathcal{A}_{i}, i=1, \ldots, n$, are commutative, then the norms $\|\cdot\|_{\mathrm{h}}$ and $\|\cdot\|_{\mathrm{ph}}$ coincide. It was shown in 20 that in general they need not even be equivalent.

Lemma 6.1. $\|\omega\|_{\text {univ }} \leq\|\omega\|_{\mathrm{ph}}$ for all $\omega \in \mathcal{A}_{1} \odot \ldots \odot \mathcal{A}_{n}$.

Proof. Let $\pi_{i}$ be a representation of $\mathcal{A}_{i}, i=1, \ldots, n$, and let $\omega=\omega_{1} \odot \omega_{2} \odot$ $\ldots \odot \omega_{n}$, where $\omega_{1} \in M_{1, k_{1}}\left(\mathcal{A}_{1}\right), \omega_{2} \in M_{k_{1}, k_{2}}\left(\mathcal{A}_{2}\right), \ldots, \omega_{n} \in M_{k_{n-1}, 1}\left(\mathcal{A}_{n}\right)$ for some $k_{1}, k_{2}, \ldots, k_{n-1} \in \mathbb{N}$.

Let $n$ be even, $\xi_{1,2} \in M_{1, l_{1}}\left(H_{1} \otimes H_{2}\right), \eta_{2,3} \in M_{l_{1}, l_{2}}\left(H_{2}^{d} \otimes H_{3}^{d}\right), \ldots, \xi_{n-1, n} \in$ $M_{l_{n-2}, 1}\left(H_{n-1} \otimes H_{n}\right)$ and

$$
\zeta=\xi_{1,2} \odot \eta_{2,3} \odot \cdots \odot \xi_{n-1, n} \in \Gamma\left(H_{1}, \ldots, H_{n}\right) .
$$


Letting $\pi=\pi_{1} \otimes \ldots \otimes \pi_{n}$, by Lemma 4.8 we have

$$
\begin{gathered}
S_{\pi(\omega)}(\zeta)=\left(\operatorname{id}_{1, k_{n-1}} \otimes \pi_{n}\right)\left(\omega_{n}^{\mathrm{t}}\right) \ldots\left(\theta_{l_{1}, l_{2}}\left(\eta_{2,3}\right)^{\mathrm{t}} \otimes I_{k_{2}}\right) \\
\times\left(\left(\operatorname{id}_{k_{1}, k_{2}} \otimes \pi_{2}\right)\left(\omega_{2}^{\mathrm{t}}\right) \otimes I_{l_{1}}\right)\left(\theta_{1, l_{1}}\left(\xi_{1,2}\right)^{\mathrm{t}} \otimes I_{k_{1}}\right)\left(\mathrm{id}_{k_{1}, 1} \otimes \pi_{1}\right)\left(\omega_{1}^{\mathrm{t}}\right)^{\mathrm{d}} .
\end{gathered}
$$

Since $\left\|\left(\operatorname{id}_{k_{m-1}, k_{m}} \otimes \pi_{m}\right)\left(\omega_{m}^{\mathrm{t}}\right)^{\mathrm{d}}\right\|=\left\|\left(\mathrm{id}_{k_{m-1}, k_{m}} \otimes \pi_{m}\right)\left(\omega_{m}\right)\right\|$, we have

$$
\begin{aligned}
\left\|S_{\pi(\omega)}(\zeta)\right\|_{\mathrm{op}} & \leq\left\|\theta_{1, l_{1}}\left(\xi_{1,2}\right)^{\mathrm{t}}\right\| \ldots\left\|\theta_{l_{n-2}, 1}\left(\xi_{n-1, n}\right)^{\mathrm{t}}\right\| \\
& \times \prod_{0 \leq i<\frac{n}{2}}\left\|\omega_{n-2 i}^{\mathrm{t}}\right\|\left\|\omega_{n-2 i-1}\right\|=\|\omega\|_{\mathrm{ph}}\|\zeta\|_{\mathrm{h}} .
\end{aligned}
$$

Now let $n$ be odd and

$$
\zeta=\eta_{1,2} \odot \xi_{2,3} \odot \cdots \odot \xi_{n-1, n} \in \Gamma\left(H_{1}, \ldots, H_{n}\right),
$$

where $\eta_{1,2} \in M_{1, l_{1}}\left(H_{1}^{\mathrm{d}} \otimes H_{2}^{\mathrm{d}}\right), \xi_{2,3} \in M_{l_{1}, l_{2}}\left(H_{2} \otimes H_{3}\right), \ldots, \xi_{n-1, n} \in M_{l_{n-2}, 1}\left(H_{n-1} \otimes\right.$ $\left.H_{n}\right)$. Using the previously obtained inequality, we have

$$
\begin{aligned}
\left\|S_{\pi(\omega)}(\zeta)\right\|_{\mathrm{op}} & =\sup _{\|\xi\| \leq 1}\left\|S_{\pi(\omega)}(\zeta)(\xi)\right\|_{H_{n}} \\
& =\sup _{\|\xi\| \leq 1}\left\|S_{\mathrm{id} \otimes \pi(1 \otimes \omega)}((1 \otimes \xi) \otimes \zeta)\right\|_{\mathcal{B}\left(\mathbb{C}^{\mathrm{d}}, H_{n}\right)} \\
& \leq\|\omega\|_{\mathrm{ph}}\|\xi\|\|\zeta\|_{\mathrm{h}} .
\end{aligned}
$$

The proof is complete.

If $H_{1}, \ldots, H_{n}$ are Hilbert spaces, we say that a net $\left\{\varphi_{\nu}\right\} \subseteq B\left(H_{1} \otimes \cdots \otimes H_{n}\right)$ converges semi-weakly to an operator $\varphi \in B\left(H_{1} \otimes \cdots \otimes H_{n}\right)$ if $\left(\varphi_{\nu} \zeta_{1}, \zeta_{2}\right) \rightarrow\left(\varphi \zeta_{1}, \zeta_{2}\right)$ for all $\zeta_{1}, \zeta_{2} \in H_{1} \odot \cdots \odot H_{n}$. Note that if the net $\left\{\varphi_{\nu}\right\}$ is bounded, then it converges semi-weakly if and only if it converges weakly.

Let $\mathcal{A}_{1} \subseteq B\left(H_{1}\right), \mathcal{A}_{2} \subseteq B\left(H_{2}\right), \ldots, \mathcal{A}_{n} \subseteq B\left(H_{n}\right)$ be $C^{*}$-algebras and $\left(\mathcal{A}_{1} \odot \mathcal{A}_{2} \odot\right.$ $\left.\ldots \odot \mathcal{A}_{n}\right)^{\sharp}$ be the linear space of all $\varphi \in \mathcal{A}_{1} \otimes \mathcal{A}_{2} \otimes \ldots \otimes \mathcal{A}_{n}$ for which there exists a net $\left\{\varphi_{\nu}\right\} \subseteq \mathcal{A}_{1} \odot \mathcal{A}_{2} \odot \ldots \odot \mathcal{A}_{n}$ converging to $\varphi$ semi-weakly (as a net of operators in $\left.B\left(H_{1} \otimes H_{2} \otimes \ldots \otimes H_{n}\right)\right)$ and such that $\sup _{\nu}\left\|\varphi_{\nu}\right\|_{\mathrm{ph}}<\infty$.

Proposition 6.2. Let $\mathcal{A}_{i} \subseteq \mathcal{B}\left(H_{i}\right), i=1, \ldots, n$, be $C^{*}$-algebras. Then $\left(\mathcal{A}_{1} \odot \cdots \odot\right.$ $\left.\mathcal{A}_{n}\right)^{\sharp} \subseteq \mathbf{M}\left(\mathcal{A}_{1}, \ldots, \mathcal{A}_{n}\right) \subseteq \mathbf{M}^{\wedge}\left(\mathcal{A}_{1}, \ldots, \mathcal{A}_{n}\right)$.

Proof. Since $\|\zeta\|_{h} \leq\|\zeta\|_{\wedge}$ for all $\zeta \in \Gamma\left(H_{1}, \ldots, H_{n}\right)$ we have $\mathbf{M}\left(\mathcal{A}_{1}, \ldots, \mathcal{A}_{n}\right) \subseteq$ $\mathbf{M}^{\wedge}\left(\mathcal{A}_{1}, \ldots, \mathcal{A}_{n}\right)$.

Let us first prove that

$$
\left(\mathcal{A}_{1} \odot \cdots \odot \mathcal{A}_{n}\right)^{\sharp} \subseteq \mathbf{M}_{\pi_{1}, \ldots, \pi_{n}}\left(\mathcal{A}_{1}, \ldots, \mathcal{A}_{n}\right),
$$

in the case where $\pi_{i}=\bigoplus_{\lambda_{i}}$ id is the sum of $\lambda_{i}$ copies of the identity representation. Let $\left\{\varphi_{\nu}\right\} \subseteq \mathcal{A}_{1} \odot \ldots \odot \mathcal{A}_{n}$ be a net converging semi-weakly to $\varphi$ and such that $D=\sup _{\nu}\left\|\varphi_{\nu}\right\|_{\mathrm{ph}}<\infty$ and $\pi=\pi_{1} \otimes \ldots \otimes \pi_{n}$. By Lemma 6.1,

$$
\left\|S_{\pi\left(\varphi_{\nu}\right)}(\zeta)\right\|_{\mathrm{op}} \leq D\|\zeta\|_{\mathrm{h}}
$$

for all $\nu$ and $\zeta \in \Gamma\left(H_{1}, \ldots, H_{n}\right)$.

Suppose first that $n$ is even. To prove that $\left\|S_{\pi(\varphi)}(\zeta)\right\|_{\mathrm{op}} \leq D\|\zeta\|_{\mathrm{h}}$, it suffices to show that the net $\left\{S_{\pi\left(\varphi_{\nu}\right)}(\zeta)\right\}$ of operators in $B\left(\widetilde{H}_{1}^{\mathrm{d}}, \widetilde{H}_{n}\right)$ converges weakly to 
the operator $S_{\pi(\varphi)}(\zeta)$ (here and in the sequel we set $\widetilde{H}_{i}=\bigoplus_{\lambda_{i}} H_{i}, i=1, \ldots, n$ ). By linearity and the uniform boundedness of the net $\left\{S_{\pi\left(\varphi_{\nu}\right)}(\zeta)\right\}$, it is sufficient to prove that

$$
\left(S_{\pi\left(\varphi_{\nu}\right)}(\zeta) x^{\mathrm{d}}, y\right) \rightarrow\left(S_{\pi(\varphi)}(\zeta) x^{\mathrm{d}}, y\right)
$$

for all $x^{\mathrm{d}}$ and $y$ which have only one non-zero entry in the corresponding direct sums of $H_{1}^{\mathrm{d}}$ and $H_{n}$, respectively.

Fix such $x^{\mathrm{d}}$ and $y$, and let $\zeta=\xi_{1,2} \otimes \eta_{2,3}^{\mathrm{d}} \otimes \ldots \otimes \xi_{n-1, n} \in \Gamma\left(\widetilde{H}_{1}, \ldots, \widetilde{H}_{n}\right)$. Then $\left(S_{\pi\left(\varphi_{\nu}\right)}(\zeta) x^{\mathrm{d}}, y\right)=\left(\pi\left(\varphi_{\nu}\right)\left(\xi_{1,2} \otimes \ldots \otimes \xi_{n-1, n}\right), x \otimes \eta_{2,3} \otimes \eta_{4,5} \otimes \ldots \otimes \eta_{n-2, n-1} \otimes y\right)$. Indeed, assuming $n=4$ for simplicity we get

$$
\begin{aligned}
\left(S_{\pi\left(\varphi_{\nu}\right)}(\zeta) x^{\mathrm{d}}, y\right) & =\left(\sigma_{\pi}\left(\varphi_{\nu}\right) \theta\left(\xi_{1,2} \otimes \xi_{3,4}\right)\left(\theta\left(\eta_{2,3}^{\mathrm{d}}\right)\right), \theta(x \otimes y)\right)_{2} \\
& =\left(\sigma_{\pi}\left(\varphi_{\nu}\right) \theta\left(\xi_{1,2} \otimes \xi_{3,4}\right), \theta\left(\theta\left(\eta_{2,3}\right) \otimes \theta(x \otimes y)\right)\right)_{2} \\
& =\left(\sigma_{\pi}\left(\varphi_{\nu}\right) \theta\left(\xi_{1,2} \otimes \xi_{3,4}\right), \theta\left(x \otimes \eta_{2,3} \otimes y\right)\right)_{2}, \\
& =\left(\pi\left(\varphi_{\nu}\right)\left(\xi_{1,2} \otimes \xi_{3,4}\right), x \otimes \eta_{2,3} \otimes y\right) .
\end{aligned}
$$

Fix $\epsilon>0$ and let $\tilde{\zeta}=\tilde{\xi}_{1,2} \otimes \tilde{\eta}_{2,3}^{\mathrm{d}} \otimes \cdots \otimes \tilde{\xi}_{n-1, n}$ be such that all norms $\left\|\xi_{1,2}-\tilde{\xi}_{1,2}\right\|,\left\|\eta_{2,3}-\tilde{\eta}_{2,3}\right\|, \ldots,\left\|\xi_{n-1, n}-\tilde{\xi}_{n-1, n}\right\|$ are smaller than $\epsilon$ and all vectors $\tilde{\xi}_{1,2}$, $\tilde{\eta}_{2,3}^{\mathrm{d}}, \ldots, \tilde{\xi}_{n-1, n}$ are finite sums of elementary tensors which have only finitely many non-zero entries in the direct sums of the corresponding Hilbert spaces. Thus, we may assume that $\tilde{\xi}_{1,2} \in H_{1}^{(k)} \odot H_{2}^{(k)}, \tilde{\eta}_{2,3} \in H_{2}^{(k)} \odot H_{3}^{(k)}, \ldots, \tilde{\xi}_{n-1, n} \in H_{n-1}^{(k)} \odot H_{n}^{(k)}$, $x^{\mathrm{d}} \in H_{1}^{(k)}$ and $y \in H_{n}^{(k)}$ for some $k \in \mathbb{N}$.

It follows from the formula above that there exists $\nu_{0}$ such that if $\nu \geq \nu_{0}$, then

$$
\left|\left(S_{\pi\left(\varphi_{\nu}\right)}(\tilde{\zeta}) x^{\mathrm{d}}, y\right)-\left(S_{\pi(\varphi)}(\tilde{\zeta}) x^{\mathrm{d}}, y\right)\right|<\epsilon
$$

On the other hand,

$$
\begin{aligned}
& \left|\left(S_{\pi\left(\varphi_{\nu}\right)}(\zeta) x^{\mathrm{d}}, y\right)-\left(S_{\pi\left(\varphi_{\nu}\right)}(\tilde{\zeta}) x^{\mathrm{d}}, y\right)\right| \\
\leq \quad & D\|x\|\|y\|\|\tilde{\zeta}-\zeta\|_{\mathrm{h}} \leq(C+\epsilon)^{n-2} D(n-1)\|x\|\|y\| \epsilon,
\end{aligned}
$$

for every $\nu$, where $C=\max \left\{\left\|\xi_{1,2}\right\|,\left\|\eta_{2,3}\right\|, \ldots,\left\|\xi_{n-1, n}\right\|\right\}$. Using Remark 4.3, we have

$$
\begin{aligned}
& \left|\left(S_{\pi(\varphi)}(\zeta) x^{\mathrm{d}}, y\right)-\left(S_{\pi(\varphi)}(\tilde{\zeta}) x^{\mathrm{d}}, y\right)\right| \\
\leq & \|\varphi\|\|x\|\|y\|\|\zeta-\tilde{\zeta}\|_{2, \wedge} \leq\|\varphi\|(C+\epsilon)^{n-2}(n-1)\|x\|\|y\| \epsilon .
\end{aligned}
$$

Thus,

$$
\begin{aligned}
& \left|\left(S_{\pi\left(\varphi_{\nu}\right)}(\zeta) x^{\mathrm{d}}, y\right)-\left(S_{\pi(\varphi)}(\zeta) x^{\mathrm{d}}, y\right)\right| \\
\leq \quad & \epsilon\left(1+(C+\epsilon)^{n-2} D(n-1)\|x\|\|y\|+\|\varphi\|(C+\epsilon)^{n-2}(n-1)\|x\|\|y\|\right)
\end{aligned}
$$

whenever $\nu \geq \nu_{0}$. It follows that the net $\left\{S_{\pi\left(\varphi_{\nu}\right)}(\zeta)\right\}$ converges weakly to $S_{\pi(\varphi)}(\zeta)$ and hence $\varphi \in \mathbf{M}_{\pi_{1}, \ldots, \pi_{n}}\left(\mathcal{A}_{1}, \ldots, \mathcal{A}_{n}\right)$.

In the case that $n$ is odd, a calculation similar to the one above shows that $\left(S_{\pi\left(\varphi_{\nu}\right)}(\zeta) x, y\right)$ is equal to

$$
\left(\pi\left(\varphi_{\nu}\right)\left(x \otimes \xi_{2,3} \otimes \ldots \otimes \xi_{n-1, n}\right), \eta_{1,2} \otimes \ldots \otimes \eta_{n-2, n-1} \otimes y\right),
$$

whenever $x \in \widetilde{H}_{1}, y \in \widetilde{H}_{n}, \zeta=\eta_{1,2}^{\mathrm{d}} \otimes \xi_{2,3} \otimes \ldots \otimes \xi_{n-1, n} \in \Gamma\left(\widetilde{H}_{1}, \ldots, \widetilde{H}_{n}\right)$, and the proof proceeds in a similar fashion. 
Now let $\pi_{1}, \ldots, \pi_{n}$ be representations of $\mathcal{A}_{1}, \ldots, \mathcal{A}_{n}$ on $H_{\pi_{1}}, \ldots, H_{\pi_{n}}$ and $\pi=\pi_{1} \otimes \ldots \otimes \pi_{n}$. Then

$$
\operatorname{rank}\left(\pi_{i}\left(a_{i}\right)\right) \leq \operatorname{rank}\left(\bigoplus_{\operatorname{dim}\left(H_{\pi_{i}}\right)} \operatorname{id}\left(a_{i}\right)\right),
$$

for all $a_{i} \in \mathcal{A}_{i}$ and $i=1, \ldots, n$. By Theorem 5.1 (i),

$$
\mathbf{M}_{\oplus_{1}}^{\bigoplus \mathrm{id}, \underset{\lambda_{2}}{\bigoplus} \mathrm{id}, \ldots, \underset{\lambda_{k}}{\bigoplus} \mathrm{id}}\left(\mathcal{A}_{1}, \ldots, \mathcal{A}_{n}\right) \subseteq \mathbf{M}_{\pi_{1}, \pi_{2}, \ldots, \pi_{k}}\left(\mathcal{A}_{1}, \mathcal{A}_{2}, \ldots, \mathcal{A}_{n}\right)
$$

The proof is complete.

Assume that $n$ is even. Then the mapping $S_{\operatorname{id}(\varphi)}$ acting on $\Gamma\left(H_{1}, \ldots, H_{n}\right)=$ $\left(H_{1} \otimes H_{2}\right) \odot\left(H_{2}^{\mathrm{d}} \otimes H_{3}^{\mathrm{d}}\right) \odot \ldots \odot\left(H_{n-1} \otimes H_{n}\right)$ can be regarded as a mapping on the algebraic tensor product

$$
H S\left(H_{n-1}, H_{n}\right) \odot H S\left(H_{n-2}, H_{n-1}\right)^{\mathrm{d}} \odot \ldots \odot H S\left(H_{1}, H_{2}\right)
$$

of the corresponding spaces of Hilbert-Schmidt operators by letting

$$
S_{\varphi}\left(\theta\left(\xi_{n-1, n}\right) \otimes \theta\left(\eta_{n-2, n-1}\right)^{\mathrm{d}} \otimes \theta\left(\xi_{n-3, n-2}\right) \otimes \ldots \otimes \theta\left(\xi_{1,2}\right)\right)=S_{\varphi}(\zeta),
$$

where $\zeta=\xi_{1,2} \otimes \eta_{2,3}^{\mathrm{d}} \otimes \xi_{3,4} \otimes \ldots \otimes \xi_{n-1, n}$. Denote the space (26) by $H S \Gamma\left(H_{1}, \ldots, H_{n}\right)$. If $\varphi$ is an elementary tensor, then Lemma 4.8 (i) shows that $S_{\operatorname{id}(\varphi)}$ is $\left(\mathcal{A}_{n}^{\prime},\left(\mathcal{A}_{n-1}^{\mathrm{d}}\right)^{\prime}, \ldots, \mathcal{A}_{2}^{\prime},\left(\mathcal{A}_{1}^{\mathrm{d}}\right)^{\prime}\right)$-modular. It follows by continuity that $S_{\operatorname{id}(\varphi)}$ is $\left(\mathcal{A}_{n}^{\prime},\left(\mathcal{A}_{n-1}^{\mathrm{d}}\right)^{\prime}, \ldots, \mathcal{A}_{2}^{\prime},\left(\mathcal{A}_{1}^{\mathrm{d}}\right)^{\prime}\right)$-modular for every $\varphi \in \mathcal{A}_{1} \otimes \cdots \otimes \mathcal{A}_{n}$. If moreover $\varphi \in \mathbf{M}_{\mathrm{id}, \ldots, \mathrm{id}}\left(\mathcal{A}_{1}, \ldots, \mathcal{A}_{n}\right)$, then $S_{\mathrm{id}(\varphi)}$ can be extended to a bounded mapping (denoted in the same way) from the algebraic tensor product

$$
\mathcal{K}\left(H_{n-1}^{\mathrm{d}}, H_{n}\right) \odot \mathcal{K}\left(H_{n-2}^{\mathrm{d}}, H_{n-1}\right)^{\mathrm{d}} \odot \cdots \odot \mathcal{K}\left(H_{1}^{\mathrm{d}}, H_{2}\right)
$$

into $\mathcal{K}\left(H_{1}^{\mathrm{d}}, H_{n}\right)$. By continuity, this extension is $\left(\mathcal{A}_{n}^{\prime},\left(\mathcal{A}_{n-1}^{\mathrm{d}}\right)^{\prime}, \ldots, \mathcal{A}_{2}^{\prime},\left(\mathcal{A}_{1}^{\mathrm{d}}\right)^{\prime}\right)$ modular.

Similarly, if $n$ is odd and $\varphi \in \mathbf{M}_{\mathrm{id}, \ldots, \mathrm{id}}\left(\mathcal{A}_{1}, \ldots, \mathcal{A}_{n}\right)$, then $S_{\mathrm{id}(\varphi)}$ can be regarded as a multilinear $\left(\mathcal{A}_{n}^{\prime},\left(\mathcal{A}_{n-1}^{\mathrm{d}}\right)^{\prime}, \ldots,\left(\mathcal{A}_{2}^{\mathrm{d}}\right)^{\prime}, \mathcal{A}_{1}^{\prime}\right)$-modular map from

$$
\mathcal{K}\left(H_{n-1}^{\mathrm{d}}, H_{n}\right) \odot \mathcal{K}\left(H_{n-2}^{\mathrm{d}}, H_{n-1}\right)^{\mathrm{d}} \odot \cdots \odot \mathcal{K}\left(H_{1}^{\mathrm{d}}, H_{2}\right)
$$

into $\mathcal{B}\left(H_{1}, H_{n}\right)$. Denote by $\mathbf{M}_{\text {id }, \ldots, \text { id }}^{c b}\left(\mathcal{A}_{1}, \ldots, \mathcal{A}_{n}\right)$ the set of all (id, ..,id)-multipliers for which the mapping $S_{\mathrm{id}(\varphi)}$ is completely bounded.

Proposition 6.3. Let $\mathcal{A}_{i} \subseteq \mathcal{B}\left(H_{i}\right), i=1, \ldots, n$, be von Neumann algebras. Then $\mathbf{M}_{\mathrm{id}, \ldots, \text { id }}^{c b}\left(\mathcal{A}_{1}, \ldots, \mathcal{A}_{n}\right) \subseteq\left(\mathcal{A}_{1} \odot \cdots \odot \mathcal{A}_{n}\right)^{\sharp}$.

Proof. Assume first that $n$ is even. For notational simplicity we assume that $H_{i}$ is separable, $i=1, \ldots, n$. Let id $: \mathcal{A}_{1} \otimes \cdots \otimes \mathcal{A}_{n} \rightarrow \mathcal{B}\left(H_{1} \otimes \cdots \otimes H_{n}\right)$ be the identity representation.

Let $\varphi \in \mathbf{M}_{\mathrm{id}, \ldots, \mathrm{id}}^{c b}\left(\mathcal{A}_{1}, \ldots, \mathcal{A}_{n}\right)$. Then $S_{\mathrm{id}(\varphi)}$ is a multilinear $\left(\mathcal{A}_{n}^{\prime},\left(\mathcal{A}_{n-1}^{\mathrm{d}}\right)^{\prime}, \ldots\right.$, $\left.\mathcal{A}_{2}^{\prime},\left(\mathcal{A}_{1}^{\mathrm{d}}\right)^{\prime}\right)$-modular mapping on

$$
\mathcal{K}\left(H_{n-1}^{\mathrm{d}}, H_{n}\right) \odot \mathcal{K}\left(H_{n-2}, H_{n-1}^{\mathrm{d}}\right) \odot \cdots \odot \mathcal{K}\left(H_{1}^{\mathrm{d}}, H_{2}\right),
$$

taking values in $\mathcal{K}\left(H_{1}^{\mathrm{d}}, H_{n}\right)$. Let $H^{\infty}=H \otimes l^{2}$, and let $I_{\infty}$ be the identity operator on $l^{2}$.

Since $S_{\operatorname{id}(\varphi)}$ is completely bounded, it extends to a completely bounded mapping, denoted in the same way, from

$$
\mathcal{K}\left(H_{n-1}^{\mathrm{d}}, H_{n}\right) \otimes_{\mathrm{h}} \mathcal{K}\left(H_{n-2}, H_{n-1}^{\mathrm{d}}\right) \otimes_{\mathrm{h}} \cdots \otimes_{\mathrm{h}} \mathcal{K}\left(H_{1}^{\mathrm{d}}, H_{2}\right)
$$


into $\mathcal{K}\left(H_{1}^{\mathrm{d}}, H_{n}\right)$. Then the second dual $S_{\mathrm{id}(\varphi)}^{* *}$ is a weak* continuous completely bounded mapping from $\mathcal{B}\left(H_{n-1}^{\mathrm{d}}, H_{n}\right) \otimes_{\sigma \mathrm{h}} \ldots \otimes_{\sigma \mathrm{h}} \mathcal{B}\left(H_{1}^{\mathrm{d}}, H_{2}\right)$ into $\mathcal{B}\left(H_{1}^{\mathrm{d}}, H_{n}\right)$ and hence gives rise to a weak* continuous completely bounded $\left(\mathcal{A}_{n}^{\prime},\left(\mathcal{A}_{n-1}^{\mathrm{d}}\right)^{\prime}, \ldots, \mathcal{A}_{2}^{\prime}\right.$, $\left.\left(\mathcal{A}_{1}{ }^{\mathrm{d}}\right)^{\prime}\right)$-modular multilinear map, denoted in the same way, from

$$
\mathcal{B}\left(H_{n-1}^{\mathrm{d}}, H_{n}\right) \times \mathcal{B}\left(H_{n-2}, H_{n-1}^{\mathrm{d}}\right) \times \cdots \times \mathcal{B}\left(H_{1}^{\mathrm{d}}, H_{2}\right)
$$

into $\mathcal{B}\left(H_{1}^{\mathrm{d}}, H_{n}\right)$.

It follows from Corollary 5.9 of [9] that there exist bounded linear operators $A_{1}: H_{1}^{\mathrm{d}} \rightarrow\left(H_{1}^{\mathrm{d}}\right)^{\infty}, A_{j}: H_{j}^{\infty} \rightarrow H_{j}^{\infty}$, if $j$ is even, $A_{j}:\left(H_{j}^{\mathrm{d}}\right)^{\infty} \rightarrow\left(H_{j}^{\mathrm{d}}\right)^{\infty}$ if $j$ is odd $(j=2, \ldots, n-1)$ and $A_{n}: H_{n}^{\infty} \rightarrow H_{n}$ such that the entries of $A_{j}$ with respect to the corresponding direct sum decomposition belong to $\mathcal{A}_{j}^{\prime \prime}=\mathcal{A}_{j}$ for even $j$ and to $\left(\mathcal{A}_{j}^{\mathrm{d}}\right)^{\prime \prime}=\mathcal{A}_{j}^{\mathrm{d}}$ for odd $j$,

$$
S_{\mathrm{id}(\varphi)}(\zeta)=A_{n}\left(\theta\left(\xi_{n-1, n}\right) \otimes I_{\infty}\right) A_{n-1}\left(\theta\left(\eta_{n-2, n-1}\right)^{\mathrm{d}} \otimes I_{\infty}\right) A_{n-2} \ldots A_{1},
$$

for all

$$
\zeta=\theta\left(\xi_{n-1, n}\right) \otimes \theta\left(\eta_{n-2, n-1}\right)^{\mathrm{d}} \otimes \ldots \otimes \theta\left(\xi_{1,2}\right) \in H S \Gamma\left(H_{1}, \ldots, H_{n}\right),
$$

and

$$
\left\|S_{\operatorname{id}(\varphi)}\right\|_{c b}=\prod_{1 \leq i \leq n}\left\|A_{i}\right\|
$$

Let $P_{m, \nu}=\left(p_{i j}^{m}\right)_{i, j=1}^{\infty}$ be the projection with $p_{i j}^{m} \in B\left(H_{m}\right)\left(\right.$ resp. $\left.p_{i j}^{m} \in B\left(H_{m}^{\mathrm{d}}\right)\right)$, $p_{i i}^{m}=I_{H_{m}}\left(\right.$ resp. $\left.p_{i i}^{m}=I_{H_{m}^{\mathrm{d}}}\right)$ if $m$ is even (resp. if $m$ is odd) and $1 \leq i \leq \nu$, and $p_{i j}^{m}=0$ otherwise.

Set $\varphi_{\nu}=A_{1}^{\mathrm{d}, \mathrm{t}} P_{1, \nu}^{\mathrm{d}} \odot P_{2, \nu} A_{2} P_{2, \nu} \odot P_{3, \nu} A_{3}^{\mathrm{d}} P_{3, \nu} \odot \ldots \odot P_{n, \nu} A_{n}$. Clearly, $\left\|\varphi_{\nu}\right\|_{\mathrm{ph}} \leq$ $\prod_{1 \leq i \leq n}\left\|A_{i}\right\|$ for each $\nu$; it hence suffices to prove that $\left\{\varphi_{\nu}\right\}$ converges semi-weakly to $\varphi$.

As $S_{\operatorname{id}\left(\varphi_{\nu}\right)}(\zeta)$ equals

$$
A_{n} P_{n, \nu}\left(\theta\left(\xi_{n-1, n}\right) \otimes I_{\infty}\right) P_{n-1, \nu} A_{n-1} P_{n-1, \nu}\left(\theta\left(\eta_{n-2, n-1}\right)^{\mathrm{d}} \otimes I_{\infty}\right) \ldots P_{1, \nu} A_{1},
$$

and $P_{l, \nu}$ converges strongly to $I_{H_{l}}$, we have that $S_{\operatorname{id}\left(\varphi_{\nu}\right)}(\zeta)$ converges weakly to $S_{\mathrm{id}(\varphi)}(\zeta)$. By the proof of Proposition 6.2 if $x^{\mathrm{d}} \in H_{1}^{\mathrm{d}}, y \in H_{n}$ and $\psi \in \mathcal{A}_{1} \otimes \ldots \otimes \mathcal{A}_{n}$, then $\left(S_{\operatorname{id}(\psi)}(\zeta) x^{\mathrm{d}}, y\right)$ equals

$$
\begin{aligned}
& \left(\sigma_{\mathrm{id}}(\psi) \theta\left(\xi_{1,2} \otimes \ldots \otimes \xi_{k-1, k}\right), \theta\left(x \otimes \eta_{2,3} \otimes \ldots \otimes \eta_{k-2, k-1} \otimes y\right)\right)_{2} \\
= & \left(\psi\left(\xi_{1,2} \otimes \ldots \otimes \xi_{k-1, k}\right), x \otimes \eta_{2,3} \otimes \ldots \otimes \eta_{k-2, k-1} \otimes y\right) .
\end{aligned}
$$

Thus $\varphi_{\nu}$ converges semi-weakly to $\varphi$ and therefore $\varphi \in\left(\mathcal{A}_{1} \odot \ldots \odot \mathcal{A}_{n}\right)^{\sharp}$, giving the inclusion $\mathbf{M}_{\mathrm{id}, \ldots, \text { id }}^{c b}\left(\mathcal{A}_{1}, \ldots, \mathcal{A}_{n}\right) \subseteq\left(\mathcal{A}_{1} \odot \ldots \odot \mathcal{A}_{n}\right)^{\sharp}$.

Now assume that $n$ is odd. In this case $S_{\operatorname{id}(\varphi)}^{* *}$ is a weak* continuous completely bounded multilinear $\left(\mathcal{A}_{n}^{\prime},\left(\mathcal{A}_{n-1}^{\mathrm{d}}\right)^{\prime}, \ldots,\left(\mathcal{A}_{2}^{\mathrm{d}}\right)^{\prime}, \mathcal{A}_{1}{ }^{\prime}\right)$-modular mapping on

$$
\mathcal{B}\left(H_{n-1}^{\mathrm{d}}, H_{n}\right) \times \mathcal{B}\left(H_{n-2}, H_{n-1}^{\mathrm{d}}\right) \times \cdots \times \mathcal{B}\left(H_{1}, H_{2}^{\mathrm{d}}\right),
$$

taking values in $\mathcal{B}\left(H_{1}, H_{n}\right)^{* *}$. Let $Q$ be the weak* continuous projection from $\mathcal{B}\left(H_{1}, H_{n}\right)^{* *}$ onto $\mathcal{B}\left(H_{1}, H_{n}\right)$. Then $Q \circ S_{\mathrm{id}(\varphi)}^{* *}$ takes values in $\mathcal{B}\left(H_{1}, H_{n}\right)$, and coincides with $S_{\mathrm{id}(\varphi)}$ on $H S \Gamma\left(H_{1}, \ldots, H_{n}\right)$. The proof now proceeds as above. 
Proposition 6.4. Let $\mathcal{A}_{i} \subseteq \mathcal{B}\left(H_{i}\right), i=1, \ldots, n$, be $C^{*}$-algebras. Then $\mathbf{M}^{\wedge}\left(\mathcal{A}_{1}, \ldots, \mathcal{A}_{n}\right) \subseteq \mathbf{M}_{\mathrm{id}, \ldots, \text { id }}^{c b}\left(\mathcal{A}_{1}, \ldots, \mathcal{A}_{n}\right)$.

Proof. Let $\varphi \in \mathbf{M}^{\wedge}\left(\mathcal{A}_{1}, \ldots, \mathcal{A}_{n}\right)$. Then there exists a constant $D>0$ such that

$$
\left\|\sigma_{\pi_{1} \otimes \ldots \otimes \pi_{n}}(\varphi)(\zeta)\right\|_{\text {op }} \leq D\|\zeta\|_{\wedge},
$$

for all $\zeta \in \Gamma\left(H_{1}, \ldots, H_{n}\right)$ and all representations $\pi_{1}, \ldots, \pi_{n}$ of $\mathcal{A}_{1}, \ldots, \mathcal{A}_{n}$, respectively.

Let $k \in \mathbb{N}$. The space $H S \Gamma\left(H_{1}^{k}, \ldots, H_{n}^{k}\right)$ is naturally isomorphic to

$$
M_{k}\left(H S\left(H_{n-1}, H_{n}\right)\right) \odot M_{k}\left(H S\left(H_{n-2}, H_{n-1}\right)^{\mathrm{d}}\right) \odot \ldots \odot M_{k}\left(H S\left(H_{1}, H_{2}\right)\right),
$$

and thus the mapping $S_{\left(\mathrm{id} \otimes 1_{k}\right) \otimes \ldots \otimes\left(\mathrm{id} \otimes 1_{k}\right)(\varphi)}$ is well-defined on the space (27). One can easily check that

$$
S_{\mathrm{id} \otimes \ldots \otimes \operatorname{id}(\varphi)}^{(k)}\left(\Xi_{n-1} \odot \ldots \odot \Xi_{1}\right)=S_{\left(\mathrm{id} \otimes 1_{k}\right) \otimes \ldots \otimes\left(\mathrm{id} \otimes 1_{k}\right)(\varphi)}\left(\Xi_{n-1} \otimes \ldots \otimes \Xi_{1}\right),
$$

where $\Xi_{i} \in M_{k}\left(H S\left(H_{i}, H_{i+1}\right)\right)$ (resp. $\Xi_{i} \in M_{k}\left(H S\left(H_{i}, H_{i+1}\right)^{\mathrm{d}}\right)$ ) if $i$ is even (resp., if $i$ is odd) and $\Xi_{i} \in M_{k}\left(H S\left(H_{i}, H_{i+1}\right)^{\mathrm{d}}\right)$ (resp. $\left.\Xi_{i} \in M_{k}\left(H S\left(H_{i}, H_{i+1}\right)\right)\right)$ if $i$ is odd (resp., if $i$ is even). If the matrices $\Xi_{i}$ are of arbitrary sizes such that the product $\Xi_{n-1} \odot \ldots \odot \Xi_{1}$ is well-defined, then they may be considered as square matrices, all of the same size, by complementing with zeros, and identity (28) will still hold. It follows that

$$
\left\|S_{\mathrm{id} \otimes \ldots \otimes \operatorname{id}(\varphi)}^{(k)}\left(\Xi_{1} \odot \ldots \odot \Xi_{n-1}\right)\right\|_{\mathrm{op}} \leq D \prod_{1 \leq i \leq n-1}\left\|\Xi_{i}\right\|_{\mathrm{op}},
$$

for all $\Xi_{1}, \ldots \Xi_{n-1}$, and hence the mapping $S_{\mathrm{id} \otimes \ldots \otimes \operatorname{id}(\varphi)}$ is completely bounded and $\varphi$ is an (id, ..., id)-multiplier.

Theorem 6.5. Let $\mathcal{A}_{i} \subseteq \mathcal{B}\left(H_{i}\right), i=1, \ldots, n$, be $C^{*}$-algebras. Then $\mathbf{M}\left(\mathcal{A}_{1}, \ldots\right.$, $\left.\mathcal{A}_{n}\right)=\mathbf{M}^{\wedge}\left(\mathcal{A}_{1}, \ldots, \mathcal{A}_{n}\right)=\left(\mathcal{A}_{1} \odot \cdots \odot \mathcal{A}_{n}\right)^{\sharp}$.

Proof. By Propositions 6.2, 6.3 and 6.4.

$$
\mathbf{M}_{\mathrm{id}, \ldots, \mathrm{id}}^{c b}\left(\mathcal{A}_{1}^{\prime \prime}, \ldots, \mathcal{A}_{n}^{\prime \prime}\right)=\left(\mathcal{A}_{1}^{\prime \prime} \odot \ldots \odot \mathcal{A}_{n}^{\prime \prime}\right)^{\sharp} .
$$

Evidently,

$$
\mathbf{M}_{\mathrm{id}, \ldots, \mathrm{id}}^{c b}\left(\mathcal{A}_{1}, \ldots, \mathcal{A}_{n}\right) \subseteq \mathbf{M}_{\mathrm{id}, \ldots, \mathrm{id}}^{c b}\left(\mathcal{A}_{1}^{\prime \prime}, \ldots, \mathcal{A}_{n}^{\prime \prime}\right) \cap\left(\mathcal{A}_{1} \otimes \ldots \otimes \mathcal{A}_{n}\right) .
$$

Applying Propositions 6.2, 6.3 and 6.4, we obtain

$$
\begin{aligned}
\left(\mathcal{A}_{1} \odot \ldots \odot \mathcal{A}_{n}\right)^{\sharp} & \subseteq \mathbf{M}\left(\mathcal{A}_{1}, \ldots, \mathcal{A}_{n}\right) \\
& \subseteq \mathbf{M}^{\wedge}\left(\mathcal{A}_{1}, \ldots, \mathcal{A}_{n}\right) \\
& \subseteq \mathbf{M}_{\mathrm{id}}^{c b}, \ldots, \mathrm{id}\left(\mathcal{A}_{1}, \ldots, \mathcal{A}_{n}\right) \\
& \subseteq \mathbf{M}_{\mathrm{id}, \ldots, \mathrm{id}}^{c b}\left(\mathcal{A}_{1}^{\prime \prime}, \ldots, \mathcal{A}_{n}^{\prime \prime}\right) \cap\left(\mathcal{A}_{1} \otimes \ldots \otimes \mathcal{A}_{n}\right) \\
& =\left(\mathcal{A}_{1}^{\prime \prime} \odot \ldots \odot \mathcal{A}_{n}^{\prime \prime}\right)^{\sharp} \cap\left(\mathcal{A}_{1} \otimes \ldots \otimes \mathcal{A}_{n}\right) .
\end{aligned}
$$

It hence suffices to show that

$$
\left(\mathcal{A}_{1}^{\prime \prime} \odot \ldots \odot \mathcal{A}_{n}^{\prime \prime}\right)^{\sharp} \cap\left(\mathcal{A}_{1} \otimes \ldots \otimes \mathcal{A}_{n}\right) \subseteq\left(\mathcal{A}_{1} \odot \ldots \odot \mathcal{A}_{n}\right)^{\sharp} .
$$

Let $\varphi \in\left(\mathcal{A}_{1}^{\prime \prime} \odot \ldots \odot \mathcal{A}_{n}^{\prime \prime}\right)^{\sharp} \cap\left(\mathcal{A}_{1} \otimes \ldots \otimes \mathcal{A}_{n}\right)$. Then there exists a net $\left\{\varphi_{\nu}\right\}_{\nu \in J} \subseteq$ $\mathcal{A}_{1}^{\prime \prime} \odot \ldots \odot \mathcal{A}_{n}^{\prime \prime}$ with $\sup _{\nu}\left\|\varphi_{\nu}\right\|_{\mathrm{ph}}<\infty$ which converges semi-weakly to $\varphi$. Write 
$\varphi_{\nu}=A_{1, \nu} \odot \ldots \odot A_{n, \nu}$, where $A_{1, \nu} \in M_{1, i_{1}}\left(\mathcal{A}_{1}^{\prime \prime}\right), A_{2, \nu} \in M_{i_{1}, i_{2}}\left(\mathcal{A}_{2}^{\prime \prime}\right), \ldots, A_{n, \nu} \in$ $M_{i_{n}, 1}\left(\mathcal{A}_{n}^{\prime \prime}\right)$.

By Kaplansky's Density Theorem for TRO's [18, for each pair $(m, \nu)$ there exists a net $\left\{A_{m, \nu, \tau(m)}\right\}_{\tau(m)} \subset M_{i_{m-1}, i_{m}}\left(\mathcal{A}_{m}\right)$ converging strongly to $A_{m, \nu}$ and such that $\left\|A_{m, \nu, \tau(m)}\right\| \leq\left\|A_{m, \nu}\right\|$ for all $\tau(m)$. Thus if $A_{\nu, \tau}=A_{1, \nu, \tau(1)} \odot A_{2, \nu, \tau(2)} \odot \ldots \odot$ $A_{n, \nu, \tau(n)}$, where $\tau=(\tau(1), \ldots, \tau(n))$, then the net $\left\{A_{\nu, \tau}\right\}_{\tau}$ converges strongly to $\varphi_{\nu}$ and $\left\|A_{\nu, \tau}\right\|_{\mathrm{ph}} \leq\left\|\varphi_{\nu}\right\|_{\mathrm{ph}}$.

Let $\mathcal{U}$ be the collection of all weak neighbourhoods of 0 of the form $\left\{S \in \mathcal{B}\left(H_{1} \otimes\right.\right.$ $\left.\left.\cdots \otimes H_{n}\right):\left|\left(S\left(\zeta_{1}^{j}\right), \zeta_{2}^{j}\right)\right|<\epsilon_{j}, j=1, \ldots, k\right\}$, where $\zeta_{1}^{j}, \zeta_{2}^{j} \in H_{1} \odot \cdots \odot H_{n}$ and $\epsilon_{j}>0, j=1, \ldots, k$. Note that $\mathcal{U}$ is directed with respect to reverse inclusion. The convergence of the net $\left\{\varphi_{\nu}\right\}_{\nu \in J}$ semi-weakly to $\varphi$ implies that for every $U \in \mathcal{U}$ there exists $\nu(U)$ such that for every $\lambda \in J$ with $\lambda \geq \nu(U)$, we have that $\varphi_{\lambda}-\varphi \in U$. The convergence of $\left\{A_{\nu, \tau}\right\}_{\tau}$ to $\varphi_{\nu}$ implies the existence of $T(\nu(U), U)$ such that for every $\tau \geq T(\nu(U), U)$, we have that $A_{\nu(U), \tau}-\varphi_{\nu(U)} \in U$. Consider the net $A_{U}=A_{\nu(U), T(\nu(U), U)}$ indexed by $\mathcal{U}$. It is easy to check that $A_{U}$ converges semiweakly to $\varphi$. The proof is complete.

Note that in Theorem 6.5 we actually proved that if $n$ is even, $\varphi \in \mathbf{M}\left(\mathcal{A}_{1}, \ldots\right.$, $\left.\mathcal{A}_{n}\right), \zeta=\xi_{1,2} \otimes \cdots \otimes \xi_{n-1, n} \in \Gamma\left(H_{1}, \ldots, H_{n}\right)$ and

$$
S_{\mathrm{id} \otimes \cdots \otimes \operatorname{id}(\varphi)}(\zeta)=A_{n}\left(\theta\left(\xi_{n-1, n}\right) \otimes I\right) \ldots\left(\theta\left(\xi_{1,2}\right) \otimes I\right) A_{1}^{\mathrm{d}},
$$

where $A_{i}$ for $i$ even (resp. $A_{i}^{\mathrm{d}}$ for $i$ odd) is a bounded block operator matrix with entries in $\mathcal{A}_{i}^{\prime \prime}\left(\operatorname{resp} .\left(\mathcal{A}_{i}^{\mathrm{d}}\right)^{\prime \prime}\right)$, then there exists a net $\varphi_{\nu}=A_{1}^{\nu} \odot A_{2}^{\nu} \odot \cdots \odot A_{n}^{\nu}$, where $A_{i}^{\nu}$ is a finite block operator matrix with entries in $\mathcal{A}_{i}$ such that $\varphi_{\nu} \rightarrow \varphi$ semi-weakly, $A_{i}^{\nu} \rightarrow A_{i}$ (resp. $A_{i}^{\nu \mathrm{d}} \rightarrow A_{i}^{\mathrm{d}}$ ) strongly for $i$ even (resp. for $i$ odd) and all operator norms $\left\|A_{i}^{\nu}\right\|,\left\|A_{i}\right\|$ are bounded by a constant depending only on $n$. A similar statement holds in the case $n$ is odd.

Denote by $\left(\mathcal{A}_{1} \odot \ldots \odot \mathcal{A}_{n}\right)^{\sim}$ the set of all $\varphi \in \mathcal{A}_{1} \otimes \ldots \otimes \mathcal{A}_{n}$ for which there exists a net $\left\{\varphi_{\nu}\right\} \subseteq \mathcal{A}_{1} \odot \cdots \odot \mathcal{A}_{n}$, such that $\sup \left\|\varphi_{\nu}\right\|_{\mathrm{ph}}<\infty$ and if $\pi_{i}$ is an irreducible representation of $\mathcal{A}_{i}, i=1, \ldots, n$, then ${ }^{\nu}\left\{\left(\pi_{1} \otimes \ldots \otimes \pi_{n}\right)\left(\varphi_{\nu}\right)\right\}$ converges semi-weakly to $\left(\pi_{1} \otimes \ldots \otimes \pi_{n}\right)(\varphi)$. Note that if $\sup _{\nu}\left\|\varphi_{\nu}\right\|_{\min }<\infty$, which holds for example when the norms $\|\cdot\|_{\mathrm{ph}}$ and $\|\cdot\|_{\mathrm{h}}$ are equivalent (see [20]), then in the definition of the space $\left(\mathcal{A}_{1} \odot \ldots \odot \mathcal{A}_{n}\right)^{\sim}$ the semi-weak convergence can be replaced by the convergence in the weak operator topology.

It follows from [21] that if $\mathcal{A}$ and $\mathcal{B}$ are commutative $C^{*}$-algebras, then $\mathbf{M}(\mathcal{A}, \mathcal{B})$ $=(\mathcal{A} \odot \mathcal{B})^{\sim}$. As a corollary of Theorem 6.5 we show that the same equality holds for an arbitrary number of arbitrary $C^{*}$-algebras, giving an answer to a problem posed in [21].

Theorem 6.6. Let $\mathcal{A}_{i}, i=1, \ldots, n$, be $C^{*}$-algebras. Then

$$
\begin{gathered}
\mathbf{M}\left(\mathcal{A}_{1}, \ldots, \mathcal{A}_{n}\right)=\mathbf{M}^{\wedge}\left(\mathcal{A}_{1}, \ldots, \mathcal{A}_{n}\right)=\left(\mathcal{A}_{1} \odot \ldots \odot \mathcal{A}_{n}\right)^{\sim} . \\
\text { Proof. Let } \pi_{1}=\bigoplus_{\pi \in \operatorname{Irr} \operatorname{Rep}\left(\mathcal{A}_{1}\right)} \pi, \ldots, \pi_{n}=\bigoplus_{\pi \in \operatorname{Irr} \operatorname{Rep}\left(\mathcal{A}_{n}\right)} \pi, \text { where } \operatorname{IrrRep}\left(\mathcal{A}_{i}\right) \text { is a }
\end{gathered}
$$
set whose elements are all inequivalent irreducible representations of $\mathcal{A}_{i}$. Then

$$
\begin{aligned}
\mathbf{M}\left(\mathcal{A}_{1}, \ldots, \mathcal{A}_{n}\right) & =\left(\pi_{1} \otimes \ldots \otimes \pi_{n}\right)^{-1}\left(\pi_{1}\left(\mathcal{A}_{1}\right) \odot \ldots \odot \pi_{n}\left(\mathcal{A}_{n}\right)\right)^{\sharp} \\
& \subseteq\left(\mathcal{A}_{1} \odot \ldots \odot \mathcal{A}_{n}\right)^{\sim} .
\end{aligned}
$$


Using arguments similar to the ones from the proof of Proposition 6.2, one can show that

$$
\left(\mathcal{A}_{1} \odot \ldots \odot \mathcal{A}_{n}\right)^{\sim} \subseteq \mathbf{M}\left(\mathcal{A}_{1}, \ldots, \mathcal{A}_{n}\right)
$$

which together with Theorem 6.5 gives the statement of the theorem.

\section{ACKNOWLEDGEMENTS}

We are grateful to V.S. Shulman for stimulating results, questions and discussions. We thank T. Itoh and A. Katavolos for providing the references 20 and 18 , respectively, and R. Levene for pointing out a mistake in an earlier version of the paper. We are indebted to the referee for a number of suggestions which helped improve the manuscript.

A part of this research was carried out when the second author was visiting Chalmers University of Technology in Göteborg, Sweden, supported by funds from Queen's University Belfast. The third author was partially supported by the Swedish Research Council, while the first and the third authors were partially supported by Engineering and Physical Sciences Research Council grant $\mathrm{EP} / \mathrm{D} 050677 / 1$.

\section{REFERENCES}

[1] W.B. Arveson, Operator Algebras and Invariant Subspaces, Annals of Mathematics 100 (1974), 433-532 MR0365167 (51:1420)

[2] C. BAdea And V.I. PAulsen, Schur multipliers and operator-valued Foguel-Hankel operators, Indiana Univ. Math. J. 50 (2001), no. 4, 1509-1522 MR1888651(2003a:47045)

[3] M.S. Birman ANd M.Z. Solomyak, Stieltjes double-integral operators. II, (Russian) Prob. Mat. Fiz. 2 (1967), 26-60

[4] M.S. Birman And M.Z. Solomyak, Stieltjes double-integral operators, III (Passage to the limit under the integral sign), (Russian) Prob. Mat. Fiz. 6 (1973), 27-53

[5] M.S. Birman AND M.Z. SolomyaK, Operator Integration, perturbations and commutators, Zap. Nauchn. Sem. Leningrad. Otdel. Mat. Inst. Steklov. (LOMI) Issled. Linein. Oper. Teorii Funktsii. 17, 170 (1989), 34-66 MR1039572 (91b:47086)

[6] M.S. Birman And M.Z. Solomyak, Double operator integrals in a Hilbert space, Integral Equations Operator Theory 47 (2003), no. 2, 131-168 MR2002663 (2004f:47029)

[7] D.P. Blecher and C. Le Merdy, Operator algebras and their modules - an operator space approach, Oxford University Press, 2004 MR2111973 (2006a:46070)

[8] D.P. Blecher And R. Smith, The dual of the Haagerup tensor product, J. London Math. Soc. (2) 45 (1992), 126-144 MR1157556 (93h:46078)

[9] E. Christensen And A.M. Sinclair, Representations of completely bounded multilinear operators, J. Funct. Anal. 72 (1987), 151-181 MR883506 (89f:46113)

[10] K.R. Davidson and V.I. Pauslen, Polynomially bounded operators, J. Reine Angew. Math. 487 (1997), 153-170 MR.1454263 (98d:47003)

[11] J. Diestel And J.J. UhL, JR., Vector measures, American Mathematical Society, Providence, $1977 \operatorname{MR} 0453964(56: 12216)$

[12] E.G. EfFros, Advances in quantized functional analysis, Proceedings of the International Congress of Mathematicians (1987), 906-916 MR.934293 (89e:46064)

[13] E.G. EfFros And Z-J. RuAn, Multivariable multipliers for groups and their operator algebras, Proceedings and Symposia in Pure Mathematics 51 (1990), Part 1, 197-218 MR.1077387 (92c:43007)

[14] E.G. Effros and Z-J. Ruan, Operator Spaces, The Clarendon Press, Oxford, 2000 MR.1793753 (2002a:46082)

[15] E.G. EFFros AND Z-J. RuAn, Operator space tensor products and Hopf convolution algebras, J. Operator Theory 50 (2003) 131-156 MR2015023 (2004j:46078)

[16] A. Grothendieck, Résumé de la théorie métrique des produits tensoriels topologiques, Boll. Soc. Mat. São Paulo 8 (1953), 1-79 MR0094682 (20:1194) 
[17] D.W. Hadwin, Nonseparable approximate equivalence, Trans. of Amer. Math. Soc. 266 (1981), no. 1, 203-231 MR613792 (82e:46078)

[18] L.A. Harris, A generalization of $C^{*}$-algebras, Proc. London Math. Soc. (3) 42 (1981), no. 2, 331-361 MR607306 (82e:46089)

[19] F. Hiai and H. Kosaki, Means of Hilbert Space Operators, Lecture Notes in Mathematics, Vol. 1820, Springer-Verlag, New York, Heidelberg, Berlin, 2003 MR2005250 (2004h:47029)

[20] Т. Ітон, The Haagerup type cross norm on $C^{*}$-algebras, Proc. Amer. Math. Soc. 109 (1990), no. 3, 689-695 MR.1014645 (90m:46096)

[21] E. Kissin and V.S. Shulman, Operator multipliers, Pacific J. Math. 227 (2006), no. 1, 109$141 \mathrm{MR} 2247875$

[22] V. PAulsen, Completely bounded maps and operator algebras, Cambridge University Press, 2002 MR 1976867 (2004c:46118)

[23] B.S. PAvLOV, Multidimensional operator integrals, Problems of Math. Anal., No. 2: Linear Operators and Operator Equations (Russian), pp. 99-122. Izdat. Leningrad. Univ., Leningrad, 1969 MR 0415371 (54:3459)

[24] V.V. Peller, Hankel operators in the perturbation theory of unitary and selfadjoint operators, Funktsional. Anal. i Prilozhen. 19 (1985), no. 2, 37-51, 96 MR800919 (87e:47029)

[25] V.V. Peller, Multiple operator integrals and higher operator derivatives, J. Funct. Anal. 233 (2006), no. 2, 515-544 MR2214586 (2008e:47056)

[26] G.PISIER, Similarity Problems and Completely Bounded Maps, Lecture Notes in Mathematics, Vol. 1618, Springer-Verlag, Berlin, New York, 2001 MR.1818047 (2001m:47002)

[27] G. PIsIER, Introduction to Operator Space Theory, Cambridge University Press, 2003 MR2006539 (2004k:46097)

[28] R. R. Smith, Completely bounded module maps and the Haagerup tensor product, J. Funct. Anal. 102 (1991), 156-175 MR.1138841 (93a:46115)

[29] N. Spronk, Measurable Schur multipliers and completely bounded multipliers of the Fourier algebras, Proc. London Math. Soc. (3) 89 (2004), no. 1, 161-192 MR2063663 (2005b:22010)

[30] M.Z. Solomjak And V.V. Stenkin, A certain class of multiple operator Stieltjes integrals. (Russian), Problems of Math. Anal., no. 2: Linear Operators and Operator Equations (Russian), pp. 122-134. Izdat. Leningrad. Univ., Leningrad, 1969 MR0438161 (55:11080)

[31] V.V. Stenkin, Multiple operator integrals. (Russian), Izv. Vysh. Uchebn. Zaved. Matematika. 4 (79) (1977), 102-115. English translation: Soviet Math. (Iz.VUZ) 21:4 (1977), 88-99 MR.0460588 (57:581)

[32] D.Voiculescu, A non-commutative Weyl-von Neumann theorem, Rev. Roumaine Math. Pures Appl. 21 (1976), 97-113 MR0415338 (54:3427)

Department of Mathematics, Chalmers Institute of Technology and University of Gothenburg, SE-412 96 Gothenburg, Sweden

E-mail address: jushenko@chalmers.se

Department of Pure Mathematics, Queen's University Belfast, Belfast BT7 1NN, UNITED Kingdom

E-mail address: i.todorov@qub.ac.uk

Department of Mathematics, Chalmers Institute of Technology and University of Gothenburg, SE-412 96 Gothenburg, Sweden

E-mail address: turowska@chalmers.se 